\title{
A Modal/WKB Inversion Method for Determining Sound Speed Profiles in the Ocean and Ocean Bottom
}

by

\author{
Kevin Donald Casey
}

B.S., United States Naval Academy (1975)

Submitted in partial fulfillment of the requirements for the degree of

OCEAN ENGINEER

at the

MASSACHUSETTS INSTITUTE OF TECHNOLOGY

and the

WOODS HOLE OCEANOGRAPHIC INSTITUTION

and

MASTER OF SCIENCE IN OCEAN ENGINEERING

at the

MASSACHUSETTS INSTITUTE OF TECHNOLOGY

June 1988

(C) Kevin D. Casey, 1988

The author hereby grants to MIT and WHOI permission to reproduce and to distribute copies of this thesis document in whole or in part.

Signature of Author

Joint Program in Oceanographic Engineering Massachusetts Institute of Technology Woods Hole Oceanographic Institution May, 1988

Certified by

Dr. George V. Frisk

Woods Hole Oceanographic Institution

Thesis Supervisor

Certified by

Dr. Arthur B. Baggeroer Massachisstto Instityte of Technology

Accepted by

W. Kendall Melville

Chairman, Joint Committee for Oceanographic Engineering, Massachusetts Institute of Technology/Woods Hole Oceanographic Institution 


\title{
A Modal/WKB Inversion Method for Determining Sound Speed Profiles in the Ocean and Ocean Bottom
}

by

\author{
Kevin Donald Casey \\ Submitted to the Massachusetts Institute of Technology- \\ Woods Hole Oceanographic Institution \\ Joint Program in Oceanographic Engineering \\ on May, 1988, in partial fulfillment of the \\ requirements for the degree of \\ OCEAN ENGINEER \\ and \\ MASTER OF SCIENCE IN OCEAN ENGINEERING
}

\begin{abstract}
Two approaches to determining the ocean sound speed profile using measured acoustic modal eigenvalues are examined. Both methods use measured eigenvalues and mode dependent assumed values of the WKB phase integral as input data and use the WKB phase integral as a starting point for relating the index of refraction to depth. Inversion method one is restricted to monotonic or symmetric sound speed profiles and requires a measurement of the sound speed at one depth to convert the index of refraction profile to a sound speed profile. Inversion method two assumes that the sound speed at the surface and the minimum sound speed in the profile are known and is applicable to monotonic profiles and to general single duct sound speed profiles. For asymmetric profiles, inversion method two gives the depth difference between two points of equal sound speed in the portion of the profile having two turning points, and in the remainder of the profile it gives sound speed versus depth directly. A numerical implementation of the methods is demonstrated using idealized ocean sound speed profiles numerical experiments used to test the performance of the inversions using noisy data. The two methods are used to determine the sediment sound speed profiles in two shallow water waveguide models, and inversion method one is used to find the sediment sound speed profile using data from an experiment performed in the Gulf of Mexico.
\end{abstract}

Thesis Supervisor: Dr. George V. Frisk

Woods Hole Oceanographic Institution 


\section{Acknowledgements}

I would like to express my gratitude to the many people who have helped me during my studies at MIT and Woods Hole, particularly my thesis advisor, Dr. George V. Frisk. Subramaniam Rajan deserves a special note of thanks for his many helpful suggetions during the course of my thesis work. Thanks also to Arthur Newhall for his help in my battles with the computer, and to Rich Signell who got me started on the computer typesetting for this thesis.

I am indebted to the U.S. Navy for providing me the opportunity to pursue my graduate studies at MIT and Woods Hole, and for funding my studies through the Office of Naval Research Fellowship Program.

Finally, I thank my wife Susan and my children Eileen and Patrick for their love and support. 


\section{Contents}

1 Introduction $\quad 10$

1.1 Background . . . . . . . . . . . . . . . . 10

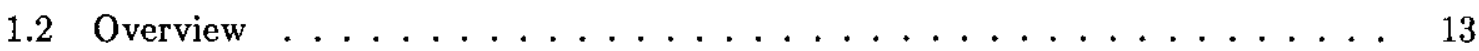

2 Normal Mode Propagation and the WKB Approximation 15

2.1 Modal Propagation . . . . . . . . . . . . . . . . . . 15

2.1 .1 The Rigid Bottom . . . . . . . . . . . . . . 16

2.1.2 Normal modes in a waveguide with a penetrable bottom . . . . . . 19

2.1.3 Analytically solvable profiles .................. 21

2.2 Rays . . . . . . . . . . . . . . . . . . . . 22

2.2 .1 The Equations of Ray Theory . . . . . . . . . . . . . . 23

2.2.2 The Connection Between Rays and Modes . . . . . . . . . . . . 24

2.3 The WKB Approximation . . . . . . . . . . . . . . . 24

$3 \quad$ The Inversion $\quad 27$

3.1 Evaluating the Phase Integral $\ldots \ldots \ldots \ldots \ldots \ldots \ldots$

3.2 Derivation of the Inversion Relations $\ldots \ldots \ldots \ldots$

3.2 .1 Derivation of inversion method $1 \ldots \ldots \ldots$

3.2 .2 Derivation of inversion method $2 \ldots \ldots \ldots 32$

3.2.3 Examples of analytically invertible profiles . . . . . . . . . . . 34

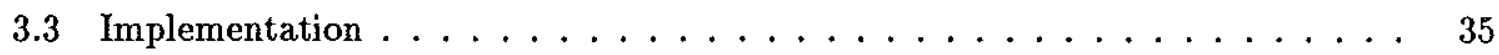

3.3.1 Implementation of inversion method $1 \ldots \ldots \ldots$

3.3.2 Implementation of inversion $\operatorname{method} 2 \ldots \ldots \ldots$ 
3.4 Application of the Inversion Methods to the Analytic Profiles . . . . . . . 39

3.4.1 Method 1 applied to the $n^{2}(z)$ linear profile . . . . . . . . . . 40

3.4.2 Method 2 applied to the $n^{2}(z)$ linear profile . . . . . . . . . 42

3.4.3 Method 1 applied to the parabolic profile . . . . . . . . . 45

3.4.4 Method 2 applied to the parabolic profile . . . . . . . . . . 45

3.5 Application of the inversion methods to a bilinear profile . . . . . . . . . 52

3.6 Performance of the inversion methods $\ldots \ldots \ldots \ldots$. . . . . . . . 61

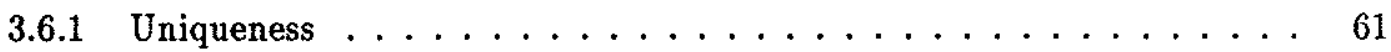

3.6.2 Resolution . . . . . . . . . . . . . . . . . 62

3.6.3 Performance of the inversion methods in the presence of noise . . . . 63

4 Application to a Shallow Water Waveguide $\quad 69$

4.1 Bottom Models and Inversions with Synthetic Data . . . . . . . . . . . 70

4.1.1 The Terrigenous Bottom Model . . . . . . . . . . . . 71

4.1 .2 The Fine Sand Bottom $\ldots \ldots \ldots \ldots \ldots$

4.2 Experimental Results . . . . . . . . . . . . . . . . . . 91

5 Conclusions $\quad 95$

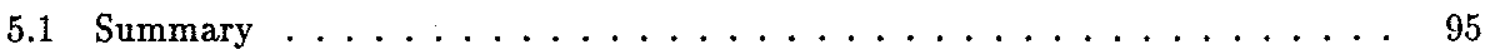

5.2 Future work . . . . . . . . . . . . . . . . . 97 


\section{List of Figures}

3-1 Region of integration for obtaining the inclusion from the WKB phase integral. 34

3-2 $F(\xi)$ for the $n^{2}(z)$ linear profile. . . . . . . . . . . . . . 41

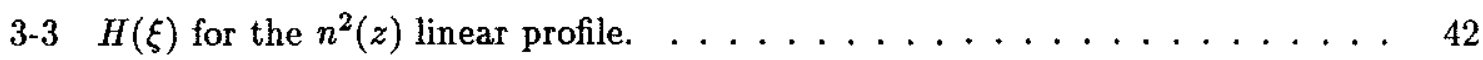

3-4 The sound speed profile for the $n^{2}(z)$ profile recovered using inversion method 1. 43

3-5 Sound speed error as a function of depth for the recovery of the $n^{2}(z)$ linear profile using inversion method $1 . \ldots \ldots \ldots 4 . \ldots \ldots$

3-6 Excursion (depth) versus sound speed for the $n^{2}(z)$ linear profile calculated using inversion method $2 \ldots \ldots \ldots \ldots$. . . . . . . . . . 46

3-7 Sound speed error versus depth for inversion method 2 applied to the $n^{2}(z)$ linear profile. ........................ 47

3-8 Depth versus sound speed for the parabolic profile recovered using inversion $\operatorname{method} 1 \ldots \ldots \ldots \ldots \ldots \ldots$

3-9 Excursion error as a function of sound speed for inversion method 1 applied to the parabolic profile. . . . . . . . . . . . . . . 49

3-10 The excursion for the parabolic profile computed using inversion method 2. . 50

3-11 Excursion error versus sound speed for inversion method 2 applied to the parabolic profile. ........................... 51

3-12 The bilinear sound speed profile model. . . . . . . . . . . . . 53

3-13 The excursion for the bilinear profile using eigenvalues for modes with an upper turning point deeper than 100 meters. . . . . . . . . . . . 54

3-14 Excursion error for the bilinear profile in the region with two turning points. 55

3-15 Excursion versus depth for the bilinear profile using eigenvalues for modes turning above 3200 meters. . . . . . . . . . . . . . 57 
3-16 Excursion versus depth for the bilinear profile. . . . . . . . . . . 58

3-17 Excursion error as a function of sound speed for the bilinear profile. . . . . . 59

3-18 Sound speed versus depth for the symmetric profile equivalent to the bilinear profile. . . . . . . . . . . . . . . . . . 60

3-19 RMS error for inversion method 1 using least squares basis splines with noisy data. . . . . . . . . . . . . . . . . . . 64

3-20 Absolute value of the maximum error for inversion method 1 using least squares basis splines with noisy data $\ldots \ldots \ldots \ldots \ldots \ldots$

3-21 RMS error for inversion method 2 using least squares basis splines with noisy

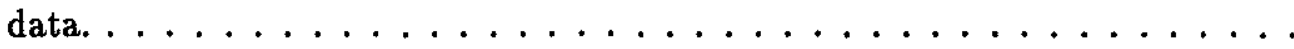

3-22 Absolute value of the maximum error for inversion method 2 using least squares basis splines with noisy data. . . . . . . . . . . . . 66

3-23 RMS error for inversion method 1 using smoothing splines with noisy data. . 66

3-24 Absolute value of the maximum error for inversion method 1 using smoothing splines with noisy data. . . . . . . . . . . . . 67

3-25 RMS error for inversion method 2 using smoothing splines with noisy data. . 67

3-26 Absolute value of the maximum error for inversion method 2 using smoothing splines with noisy data. . . . . . . . . . . . . . . 68

3-27 Sound speed error for the $n^{2}(z)$ linear profile with mode 25 eigenvalue removed, and higher modes incorrectly identified. . . . . . . . . . . .

4-1 Experimental configuration for measuring the eigenvalues in a shallow water waveguide ............................... 70

4-2 Shallow water waveguide model 1 ; terrigenous bottom. . . . . . . . 73

4-3 Inversion results for waveguide model 1 ; method $1 \ldots \ldots . \ldots 74$

4-4 Depth error for waveguide $\operatorname{model} 1 ; \operatorname{method} 1 \ldots \ldots \ldots$

$4-5$ Inversion results for waveguide model $1 ; \operatorname{method} 2 \ldots \ldots 75$

4-6 Depth error for waveguide $\operatorname{model} 1 ; \operatorname{method} 2 \ldots \ldots$. . . . . . . 76

4-7 Inversion results for waveguide model 1 with a low velocity zone. . . . . . . 77

4-8 Inversion results for waveguide model 1 with a low velocity zone using the correct reference sound speed. . . . . . . . . . . . . . 78 
4-9 The shallow water waveguide model with a fine sand bottom. . . . . . . . 80

4-10 Inversion results for waveguide model 2 ; method 1 (least squares splines). . . 81

4-11 Depth error for waveguide model 2; method 2 (least squares splines) . . . . 82

4-12 Inversion results for waveguide model 2; method 1 (smoothing splines). . . . . 83

4-13 Depth error for waveguide model 2 ; method 1 (smoothing splines) . . . . . . 84

4-14 Inversion results for waveguide model 2; inversion method 2 (least squares

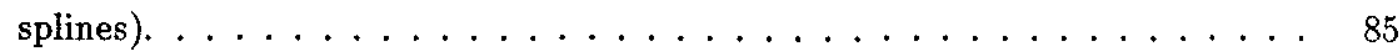

4-15 Depth error for waveguide model 2; method 2 (least squares splines). . . . . 86

4-16 Inversion results for waveguide model 2 ; method 2 (smoothing splines). . . . 87

4-17 Depth error for waveguide model 2 ; method 2 (smoothing splines). . . . . . 88

4-18 Inversion results for waveguide model 2 including reflection effects. . . . . . . 89

4-19 Inversion results for waveguide model 2 using all propagating eigenvalues. . . 90

4-20 Sound speed profile for the Corpus Christi area based on geologic information. 92

4-21 Initial Corpus Christi area sediment sound speed profile estimate. . . . . . . . 93

4-22 Final Corpus Christi area sediment sound speed profile compared to other results for the area. . . . . . . . . . . . . . . . . . 94 


\section{List of Tables}

4.1 Experimentally measured eigenvalues for the Corpus Christi site. . . . . . . 91 


\section{Chapter 1}

\section{Introduction}

As sound propagates through a medium, the sound field is affected by the medium through which the sound is passing, and by the nature and physical properties of the medium's boundaries. This relationship between the sound field and the medium may be used to predict the properties of the sound field as the sound propagates through a medium with known properties (the forward problem) or to infer the properties of a medium from measurements of the sound field after propagation through the medium (the inverse problem). Using sound we may thus able to measure the properties of media which might otherwise be difficult to probe. In this thesis we present a nonperturbative inverse method based on the WKB approximation to the sound field which uses the values of the modal eigenvalues as input data.

\subsection{Background}

In ocean acoustic tomography, linear inverse techniques are applied to measurements of perturbations in the sound travel time which result from sound speed variations along the signal path. These sound speed variations are then related to the oceanic processes causing the sound speed variations. Acoustic tomography techniques have been used to study ocean features such as mesoscale eddies [12], internal waves [17], and barotropic motions [9]. While these processes may be studied in other ways, tomography has the advantage of being able to provide frequent measurements of the ocean properties over large areas. Equivalent mea- 
surements made by other means would be prohibitively expensive. In these tomographic studies, measurements are made using sound travelling over paths which do not involve interaction with either the ocean surface or bottom, however; sound which does interact with the boundaries can be used to study the boundaries. James Miller in his ScD thesis [34], for example, uses tomographic techniques to obtain estimates of the sea surface spectra, and acoustic signals generated in the water column have been used extensively in marine seismology to study crustal structure and properties [16]. For studying sound propagation in the ocean, the properties of the ocean bottom are needed on a much finer scale than provided by marine seismological measurements, and the region of interest, rather than extending to several kilometers depth, is limited to the top few hundred meters of the sediments at most frequencies of interest. Frisk et al. [19] use amplitude versus range data obtained using a deep-towed pulsed CW source and two receivers moored near the bottom to infer geoacoustic models of the upper sediment layers in deep water. The model is derived from the measured data using an iterative forward modelling technique. This method relies on time gating to separate the bottom reflected signal from the surface reflected signal. In shallow water, time gating method is not practical because the time differences between signals reflected from the surface and signals reflected from the bottom are too small. Instead, a horizontal array is used to measure the steady state sound field which is then decomposed into its eigenfunctions. Using the eigenvalues of the propagating sound field, Rajan et al. [37] obtain the sediment properties using a perturbative approach.

Rather than using a perturbative technique, we use a WKB phase integral based inverse method to obtain the sound speed profile using as input data the eigenvalues of the sound field in the ocean acoustic waveguide. The method may be used to find the sound speed profile in the water column and in the upper layers of sediments. Although the sediments have enough rigidity to transmit shear waves, and we expect compressional to shear wave conversion to take place at layer interfaces, Fryer [23] indicates that effects of compressional to shear conversion resulting from shear speed gradients within the sediment column is small above $20 \mathrm{~Hz}$. Since we are interested in the upper sediment layers where shear wave speed and shear wave speed gradients are small, we will treat the sediments as a fluid extension of the water column and neglect all shear effects. This will enable us to determine the compressional sound speed 
profile in the sediments in that portion of the sediments affecting sound propagation at the frequencies of interest (the standard frequency used in the examples will be $220 \mathrm{~Hz}$ ). These methods will not be able to distinguish the low velocity zone that commonly occurs at the water/sediment interface for bottom materials such as pelagic clay [25]. This inability to find low velocity regions results because the inversions are determining ray turning depths and rely on sound energy being turned by an increasing sound speed within a region in order to find the sound speed/depth relationship in that region. The sound does not turn within a low velocity zone. Using an approach suggested by Vassell to study the index of refraction in optical waveguides [43], we relate the normal mode eigenvalues to the sound speed profile via the WKB phase integral

$$
k_{0} \int_{z_{1}}^{z_{2}}\left[n^{2}(z)-\sin ^{2} \theta_{n}\right]^{1 / 2} d z
$$

Here $k_{0}$ is the wavenumber given by $\omega / c_{0}$ where $\omega$ is the frequency and $c_{0}$ is the reference sound speed, $\theta_{n}$ is the local propagation angle corresponding to the $n$th mode, and $z_{1}$ and $z_{2}$ are the turning depths for the mode. The phase integral will be treated as a function of the new variable $\xi=\sin ^{2} \theta_{n}$. The value of this integral may be estimated based on the mode number and the type of boundary interactions experienced by the mode. For example, the phase integral for the third mode will assume a different value in an environment giving two turning points than it will in an environment where the mode interacts with the surface and has only a lower turning point.

There are two methods for obtaining the index of refraction as a function of depth. The first method (inversion method 1) follows Vassell's treatment and can be used for sound speed profiles that increase monotonically with depth or are symmetric about a depth of minimum sound speed. In this method, the WKB phase integral equation is differentiated to arrive at an Abel integral equation which can be used to relate depth to index of refraction. From knowledge of the sound speed at one depth, we can determine the sound speed profile. If the sound speed profile is symmetric rather than monotonic, the inversion gives the sound speed profile below the depth of symmetry, and the symmetry of the profile is used to generate the upper portion of the profile.

The second method (inversion method 2) is based on a method used in quantum mechanical scattering for determining molecular potentials and is referred to in the quantum 
mechanical context as the JWKB or semi-classical approximation [10],[44]. Now the WKB phase integral is transformed to the form

$$
I(\xi)=\int_{z_{1}}^{z_{2}}\left[n^{2}(z)-\xi\right] d z
$$

and $I(\xi)$ (referred to as the inclusion) is differentiated to produce the excursion which is the difference in depth between two points of the sound speed profile having the same sound speed. In general there is insufficient information to translate this to a sound speed profile; however, if the profile is monotonic or symmetric, this method gives the sound speed profile directly. For an asymmetric profile in which some modes have two turning points and some have one turning point, inversion method 2 will give a sound speed profile for the range of sound speeds having a single turning depth since the surface is essentially the upper turning depth.

Munk and Wunsch [35] derive an Abel transform based inversion method along the lines of our inversion method 1 ; however, they deal strictly with applications to the water column, and they approach the asymmetric case in a different fashion. Much of the previous work in inverse methods for finding the sound speed profile in the ocean and the sediments has used perturbation methods largely because the techniques of linear perturbation theory provide a means to compute estimates of errors and depth resolution [33]. With the methods discussed here, we can only give qualitative arguments concerning resolving power and numerical examples illustrating accuracy of the methods and their performance in a noisy environment. The main advantage with our methods is that they do not require an initial estimate of the sound speed profile.

\subsection{Overview}

Chapter 2 contains a review of the essentials of normal mode theory, ray theory, and WKB theory in the context of underwater acoustics. Since the inversion methods presented here are based on the normal mode eigenvalues as input data and use the WKB phase integral as a starting point for relating the index of refraction to the eigenvalues, an understanding of normal modes and the WKB approximation is important. Ray theory provides a means for building a conceptual picture of the inversion process through the ray theory view of the 
turning point.

Chapter 3 contains the bulk of the thesis. We start by grouping normal modes according to the boundary interactions and the number of turning points (we do not allow for multiple duct profiles), and then evaluating the WKB phase integral for each of the four types of mode. These phase integral values and the associated eigenvalues provide the input data used in generating a profile dependent functional relationship for the phase integral. The Abel integral equation based inversion relations for the two inversion methods are then derived. Following a brief description of the spline methods used in the computations, we discuss the numerical implementation of the two inversion methods. Both inversion methods are applied to the $n^{2}(z)$ linear profile, the parabolic profile, and a bilinear profile to illustrate their capabilities under ideal conditions. Using the $n^{2}(z)$ profile, we then demonstrate the performance of the inversion methods using data contaminated with random noise.

In chapter 4 we apply inversion method 1 to the problem of determining the sediment sound speed profile in a shallow water waveguide by treating the sediment as a fluid extension of the water column. Two synthetic models are used to test the inversions: (1) a waveguide with a terrigenous bottom, and (2) a waveguide with a fine sand bottom. We then apply the inversion method to data obtained in an experiment performed in the Gulf of Mexico.

Chapter 5 summarizes the results of the work and presents ideas for future work. 


\section{Chapter 2}

\section{Normal Mode Propagation and the WKB Approximation}

\subsection{Modal Propagation}

The input data for the inverse method considered in this work are the measured horizontal wavenumbers or eigenvalues for the modal description of sound propagation in the medium, and a brief review of normal mode propagation is given here to emphasize the normal mode ideas important to the inversion. Normal mode theory provides a 'full wave' solution to the wave equation in the ocean waveguide which takes into account the properties of the medium in the waveguide, frequency effects, and the effects of the properties of the waveguide boundaries. The degree to which the solution to the wave equation provided by modal theory realistically describes acoustic propagation in the ocean depends on the accuracy to which the medium properties and boundary conditions are modeled. We will use models of varying sophistication to illustrate various aspects of the normal mode description of sound propagation pertinent to the inverse method.

We model the ocean as a waveguide consisting of a water column bounded above by the air-ocean interface where the acoustic pressure is zero, and below by a sediment bottom which will be considered a semi-infinite half-space. The sound speed is $c(z)$ and the density $\rho$ are taken to be piecewise constant throughout most of the work. The following simplifying assumptions are used throughout: 
1. The source is a monochromatic point source.

2. Cylindrical symmetry about the vertical axis through the source.

3. The medium properties vary only in depth, i.e. the problem is range independent. Included here is the assumption that the water depth is constant.

4. No scattering from rough surfaces or inhomogeneities in the medium.

5. The bottom is a fluid which may effectively be treated as an extension of the water column.

With the assumption of cylindrical symmetry, the spatial part of the acoustic pressure $p\left(r, z, z_{0}\right)$, due to a point source located at $r=0$ and $z=z_{0}$ with harmonic time dependence $\exp (-i \omega t)$, satisfies the inhomogeneous Helmholtz equation:

$$
\left[\frac{1}{r} \frac{\partial}{\partial r}\left(r \frac{\partial}{\partial r}\right)+\frac{\partial^{2}}{\partial z^{2}}+k^{2}(z)\right] p\left(r, z, z_{0}\right)=-2\left[\frac{\delta(r)}{r}\right] \delta\left(z-z_{0}\right)
$$

where $\omega$ is the frequency and $k(z)=\omega / c(z)$.

To complete the problem, appropriate boundary conditions must also be specified. At any interface where there is a sudden change in the medium properties (the water-sediment interface for example) the boundary conditions of continuity of pressure and continuity of normal particle velocity must be satisfied. In the range equation the radiation condition that there are no waves propagating inward from infinity will be used.

\subsubsection{The Rigid Bottom}

We start by considering the simplest model of the ocean waveguide consisting of a layer of water with sound speed $c(z)$, which may be a function of depth, and constant density, $\rho_{0}$. The layer is bounded above by a pressure release-surface (the air-water interface) and below by a rigid bottom. The symmetry and range independence of the problem lead naturally to use of separation of variables in solving the problem. Letting

$$
p\left(r, z, z_{0}\right)=\phi(z) \psi(r)
$$


and substituting this into the homogeneous Helmholtz equation leads to the separated equations

$$
\frac{d^{2} \phi(z)}{d z^{2}}+\gamma^{2} \phi(z)=0
$$

and

$$
\frac{d^{2} \psi(r)}{d r^{2}}+\frac{1}{r} \frac{d \psi(r)}{d r}+k_{r}^{2} \psi(r)=0
$$

where $-k_{r}^{2}$ is a separation constant, and

$$
\gamma^{2}=k^{2}(z)-k_{r}^{2}
$$

The total acoustic pressure vanishes everywhere on the pressure-release surface and a sound wave incident on the surface is completely reflected with a plane wave undergoing a. $\pi$ phase shift. The rigid boundary condition is characterized by the condition that the normal particle velocity vanishes on the surface resulting in complete reflection. For an incident plane wave there will be no phase shift upon reflection from the rigid boundary. In this model, the depth function $\phi(z)$ satisfies the boundary conditions

$$
\phi(0)=0
$$

for the pressure-release surface and

$$
\frac{d \phi(h)}{d z}=0
$$

for the rigid bottom.

Equation (2.3) only has solutions for certain values of the parameter $\gamma$ (the eigenvalues). Because this is a Sturm-Liouville problem, the set of eigenfunctions $\phi_{i}(z), i=1,2,3, \ldots$, forms a complete orthonormal set of square integrable functions on the interval $0<z<h$ implying that [45]

$$
\int_{0}^{h} \frac{\phi_{i}(z) \phi_{j}(z)}{\rho_{0}}= \begin{cases}0 & i \neq j \\ 1 & i=j\end{cases}
$$

where the $1 / \rho_{0}$ weighting is included in analogy to the results in problems where the density is a function of depth.

Up to this point we have allowed the sound speed to be any function of the depth $z$. For definiteness in illustrating the solution of the problem, we now specify $c(z)=$ constant. The 
boundary conditions lead to the equation

$$
\gamma_{n}=\frac{(2 n-1) \pi}{2 h}
$$

for the vertical wavenumber $\gamma_{n}$. Using a density $\rho_{0}=1$ and normalizing gives for the eigenfunctions

$$
\phi_{n}(z)=\sqrt{\frac{2}{h}} \sin \left[\left(k^{2}-k_{r_{n}}^{2}\right)^{1 / 2} z\right]
$$

where the horizontal wavenumbers $k_{r_{n}}$ are given by

$$
k_{r_{n}}^{2}=k^{2}-\left[\frac{(2 n-1) \pi}{2 h}\right]^{2} \text {. }
$$

Expanding the solution of equation (2.1) in terms of the eigenfunctions

$$
p\left(r, z, z_{0}\right)=\sum_{n=1}^{\infty} \phi_{n}(z) \psi_{n}(r),
$$

substituting expansion into equation (2.1), and using the orthonormality of the depth functions gives

$$
\frac{d^{2} \psi_{n}(r)}{d r^{2}}+\frac{1}{r} \frac{d \psi_{n}(r)}{d r}+k_{r_{n}}^{2} \psi_{n}(r)=-\frac{2}{r} \delta(r) \phi_{n}\left(z_{0}\right)
$$

which is a Bessel equation of order zero for which the solution is a linear combination of Hankel functions. We use the radiation condition that there are no waves propagating inward from infinity to eliminate the term involving $H_{0}^{(2)}$, and the solution to the radial equation is

$$
\psi_{n}(r)=i \pi \phi_{n}\left(z_{0}\right) H_{0}^{(1)}\left(k_{r_{n}} r\right)
$$

The solution to equation (2.1) is then expressed as a sum of the normal modes

$$
p\left(r, z, z_{0}\right)=\frac{2 \pi i}{h} \sum_{n}^{\infty} \sin \left(\gamma_{n} z\right) \sin \left(\gamma_{n} z_{0}\right) H_{0}^{(1)}\left(k_{r_{n}} r\right)
$$

At distances of more than a few wavelengths from the source, we can use the asymptotic approximation to the Hankel function [2]

$$
H_{0}^{(1)}\left(k_{r} r\right) \sim \sqrt{\frac{2}{\pi k_{r} r}} \exp \left[i\left(k_{r} r-\pi / 4\right)\right]
$$

The normal mode solution of equation ( 2.1) given by equation (2.14) describes the sound field in terms of a sum of travelling cylindrical waves propagating outward from the 
origin. Each of these modes is a standing wave in the vertical direction with a distinct depth distribution of pressure described by the eigenfunction $\phi(z)$ and the vertical wavenumber $\gamma_{n}$. Although the summation of equation (2.14) is infinite, for a sufficiently large mode number $\mathrm{N}$, the horizontal wavenumber will become imaginary, and the solution will rapidly decay with range. For a receiver located sufficiently far from the source, the sound field will be adequately described by a finite sum of propagating modes. It is these horizontal wavenumbers from this description of the sound field that will provide the input information for the inversion method described in the next chapter.

\subsubsection{Normal modes in a waveguide with a penetrable bottom}

If the bottom of the waveguide is not a rigid reflector, some of the sound energy will penetrate into the bottom, and the solution to the Helmholtz equation must be found both in the water and in the bottom. The approach taken here is different from the previous section in that the Hankel transform is used to obtain the solution; however, the essential ideas related to the propagating modes remain the same.

Starting once again with the inhomogeneous Helmholtz equation (equation (2.1)), we introduce the zero order Hankel transform pair

$$
\begin{gathered}
p\left(r, z, z_{0}\right)=\int_{0}^{\infty} \tilde{p}\left(k_{r}, z, z_{0}\right) J_{0}\left(k_{r} r\right) k_{r} d r \\
\tilde{p}\left(k_{r}, z, z_{0}\right)=\int_{0}^{\infty} p\left(r, z, z_{0}\right) J_{0}\left(k_{r} r\right) r d r
\end{gathered}
$$

where $J_{0}\left(k_{r} r\right)$ is the Bessel function of order zero.

Multiplying equation (2.1) by $J_{0}\left(k_{r} r\right) r d r$ and integrating from 0 to $\infty$ (see [3] for details of the integration), we find that the transform variable (or depth dependent Green's function) must satisfy $[20]$

$$
\left(\frac{d^{2}}{d z^{2}}+k^{2}(z)-k_{r}^{2}\right) \tilde{p}\left(k_{r}, z, z_{0}\right)=-2 \delta\left(z-z_{0}\right) .
$$

Let $\tilde{p}_{1}$ and $\tilde{p}_{2}$ be linearly independent solutions of equation (2.18) chosen such that $\tilde{p}_{1}$ satisfies the surface boundary condition, and $\tilde{p}_{2}$ satisfies the bottom boundary condition. Then the transform function $\tilde{p}\left(k_{r}, z\right)$ is given by [20]

$$
\tilde{p}\left(k_{r}, z, z_{0}\right)=\frac{-2 \tilde{p}_{1}\left(k_{r}, z\right) \tilde{p}_{2}\left(k_{r}, z_{0}\right)}{W\left(z_{0}\right)} ; 0 \leq z \leq z_{0}
$$




$$
\tilde{p}\left(k_{r}, z, z_{0}\right)=\frac{-2 \tilde{p}_{1}\left(k_{r}, z\right) \tilde{p}_{2}\left(k_{r}, z_{0}\right)}{W\left(z_{0}\right)} ; \quad z_{0} \leq z
$$

where $W\left(z_{0}\right)$ is the Wronskian given by

$$
W\left(z_{0}\right)=\tilde{p}_{2}\left(k_{r}, z_{0}\right) \tilde{p}_{1}^{\prime}\left(k_{r}, z_{0}\right)-\tilde{p}_{2}^{\prime}\left(k_{r}, z_{0}\right) \tilde{p}_{1}\left(k_{r}, z_{0}\right)
$$

where the prime indicates differentiation with respect to $\mathrm{z}$.

Next the Bessel function is expressed in terms of Hankel functions as

$$
J_{0}\left(k_{r} r\right)=\frac{1}{2}\left[H_{0}^{(1)}\left(k_{\tau} r\right)+H_{0}^{(2)}\left(k_{r} r\right)\right]
$$

and use the relation

$$
H_{0}^{(2)}\left(e^{-i \pi} k_{r} r\right)=-H_{0}^{(1)}\left(k_{r} r\right)
$$

to allow the range of integration for the transform integrals to be extended over the whole real $k_{r}$ axis from $-\infty$ to $\infty$ when evaluating the transform integral for $p(r, z)$. The solution is then found by contour integration methods [3], and may be expressed as the sum of a discrete and a continuous portion given by [20]

$$
p(r, z)=i \pi \sum \phi_{n}\left(z_{0}\right) \phi_{n}(z) H_{0}^{(1)}\left(k_{r_{n}} r\right)+I(r)
$$

where the eigenfunctions and eigenvalues satisfy

$$
\left(\frac{d^{2}}{d z^{2}}+k^{2}(z)-k_{r_{n}}^{2}\right) \phi_{n}(z)=0
$$

along with the surface and bottom boundary conditions.

The part of the solution representing the continuum $(I(r)$ or the branchline contribution) is rapidly attenuated with range and will be neglected, since we are only concerned with measurements made more than a few wavelengths from the source. We note that the discrete portion of the solution is of the same form as for the rigid bottom case, and that the eigenvalues have the same interpretation, although they will take on different values since the sound is propagating in both media, and the characteristic equation will be different.In general the lowest medium will be taken as a half space extending to infinity and a radiation condition will be imposed resulting in a solution that decays with depth in the halfspace. 


\subsubsection{Analytically solvable profiles}

We next consider two specific sound speed profiles in order to illustrate the concept of turning depth. A turning depth is a depth at which the vertical wavenumber vanishes, leading to a fundamental change in the behaviour of the solution to the Helmholtz equation. In the region where the vertical wavenumber is greater than zero, the solution is oscillatory, while in the region for which the vertical wavenumber is less than zero, the solution will decay with depth. Depending on the sound speed structure, these turning points may be located in either the water column or in the sediment. The sound speed profiles we will consider are: (1) the square of the index of refraction a linear function of depth, and (2) the square of the index of refraction parabolic. In both these cases solution of the depth equation gives closed form expressions for the eigenvalues. Additionally, we will later find that these profiles may be inverted analytically by the methods presented here, and consequently they will be the standard examples for illustrating results and verifying the numerical methods.

\section{The $n^{2}(z)$ linear waveguide}

For simplicity we consider a semi-infinite ocean with a pressure-release surface and an index of refraction which satisfies the relation

$$
n^{2}(z)=1-a z
$$

In lieu of a bottom boundary condition, we apply a radiation in depth requiring that the solution remain finite at infinity.

Proceeding as for the penetrable bottom case, the homogeneous part of equation (2.18) becomes

$$
\frac{d^{2} \tilde{p}\left(k_{r}, z, z_{0}\right)}{d z^{2}}+\left[k_{0}^{2}(1-a z)-k_{r}^{2}\right] \tilde{p}\left(k_{r}, z, z_{0}\right)=0
$$

Making the change of variables [8]

$$
H=\left(a k_{0}^{2}\right)^{-1 / 3} ; \quad t_{0}=H^{2}\left(k_{r}^{2}-k_{0}^{2}\right) ; \quad t=t_{0}+z / H,
$$

equation (2.27) is transformed into the Airy equation

$$
\frac{d^{2} \tilde{p}(t)}{d t^{2}}=t \tilde{p}(t)
$$


which has as solutions the Airy functions $A i(t)$ and $B i(t)$ [2]. (Here functional dependence other than dependence on $t$ has been surpressed.) We eliminate $B i(t)$ in order to satisfy the radiation condition, and the eigenvalues are found using the pressure release condition which requires $A i\left(t_{0}\right)=0$ from which we see that $t_{0}=-y_{n}$ where the $y_{n}$ are the zeros of the Airy function. With $t_{n}=z / H-y_{n}$ and $t_{0 n}=z_{0} / H-y_{n}$ the sound field is

$$
p(r, z)=\frac{i \pi}{H} \sum_{n} \frac{A i\left(t_{n}\right) A i\left(t_{0 n}\right) H_{0}^{(1)}\left(k_{r} r\right)}{\left[A i^{\prime}\left(-y_{n}\right)\right]^{2}} .
$$

The eigenvalues are given by

$$
k_{\tau_{n}}^{2}=k_{0}^{2}-y_{n} / H^{2}
$$

\section{The parabolic profile}

In considering the parabolic profile, we take the ocean as infinite in the vertical direction so that there are no boundaries, and the radiation condition is used to give a solution that remains finite at $\pm \infty$. The index of refraction satisfies

$$
n^{2}(z)=1-\alpha^{2} z^{2}
$$

and the homogeneous part of equation $(2.18)$ is

$$
\frac{d^{2} \tilde{p}\left(k_{r}, z, z_{0}\right)}{d z^{2}}+\left[k_{0}^{2}\left(1-\alpha^{2} z^{2}\right)-k_{r}^{2}\right] \tilde{p}\left(k_{r}, z, z_{0}\right)=0
$$

The solution to this are given in terms of Hermite polynomials as [42]

$$
\tilde{p}\left(k_{r}, z, z_{0}\right)=\left(2^{n} n !\right)^{-1 / 2}\left(\alpha k_{0} / \pi\right)^{1 / 4} e^{-\frac{1}{2} \alpha k_{0} z^{2}} H_{n}\left(\sqrt{\alpha k_{0}} z\right)
$$

where the $H_{n}(\cdot)$ are the Hermite polynomials. The eigenvalue condition gives

$$
k_{n}=k_{0}\left(1-\alpha(2 n+1) / k_{0}\right)^{1 / 2} ; \quad n=0,1,2, \ldots
$$

\subsection{Rays}

Ray theory is a geometric solution of the wave equation which is correct for high frequencies and provides a simple physical description of the solution in terms of the paths along which the acoustic energy is refracted. The ray approximation is widely used for studying sound 
propagation in underwater acoustics, and provides a convenient method for visualizing the propagation paths of the sound energy and the depth of penetration of the sound energy into the bottom. We will briefly illustrate the connection between the normal mode view and the ray picture to the extent that it helps in understanding the inversion technique. A number of references are available which provide detailed treatments of ray theory in underwater acoustics [7],[6].

\subsubsection{The Equations of Ray Theory}

The equations for ray acoustics [7] are obtained from the Helmholtz equation in vector form using pressure as the variable

$$
\nabla^{2} p+k^{2}(\vec{x}) p=0
$$

where $\vec{x}$ is the position vector and $k(\vec{x})=\omega / c(\vec{x})$, by taking the solution to be

$$
p(\vec{x})=A(\vec{x}) \exp \left(i k_{0} S(\vec{x})\right)
$$

Here $k_{0}=\omega / c_{0}$ where $c_{0}$ is a reference sound speed such as the minimum sound speed in the waveguide (or the sound speed at the source), $A(\vec{x})$ is the amplitude of the sound waves, and $k_{0} S(\vec{x})$ is the phase, with the function $S(\vec{x})$ referred to as the eikonal.

Substituting the assumed solution (equation (2.37)) into the Helmholtz equation gives

$$
\nabla^{2} A+i k_{0}\left(2 \nabla A \cdot \nabla S+A \nabla^{2} S\right)+k_{0}^{2} A\left[n^{2}(\vec{x})-(\nabla S)^{2}\right]=0
$$

The equations of ray theory are obtained from equation (2.38) in the limit as $k_{0} \rightarrow \infty$ by equating the real and imaginary terms to 0 , and neglecting the $\nabla^{2} A$ compared to the real term containing $k_{0}^{2} A$ (that is we assume $\nabla^{2} A / A \ll k_{0}^{2}$ ). The resulting equations are the eikonal equation

$$
(\nabla S)^{2}=n^{2}(\vec{x})
$$

and the transport equation

$$
2 \nabla A \cdot \nabla S+A \nabla S=0 .
$$

The eikonal equation defines the geometry of the rays which are lines perpendicular to the surfaces of constant phase, that is $S(\vec{x})=$ constant . These ray trajectories can be computed to determine the path of the sound energy. 


\subsubsection{The Connection Between Rays and Modes}

C.T. Tindle and K.M. Guthrie [24] [41] have illustrated the connection between normal mode theory and the ray theory approximation. Considering a deep water sound speed profile with a minimum sound speed at some depth below the surface, then for a given mode number $n$, a ray with the same turning point depths as mode $n$ can be defined by

$$
\frac{c_{0}}{\sin \theta_{0}}=\frac{\omega}{k_{r_{n}}}
$$

where the angle $\theta_{0}$ is the angle of incidence for the ray as it crosses the channel axis (i.e. the sound speed minimum), and $k_{r_{n}}$ is the horizontal wavenumber for mode $n$. The ray effects are duplicated by summing over a group of modes, and each group will manifest itself as an energy pulse travelling along the ray path which corresponds to the ray defined by the angle $\theta_{0}$ for the central mode in the group of modes. Thus we can construct a ray corresponding to the group of modes; however, this ray will not necessarily be the same as the physical ray path along which the sound energy actually travels between the source and receiver. By using this view of rays as the result of modal interference, we can construct a picture of the path followed by the sound, and its turning depths within the framework of normal modes.

\subsection{The WKB Approximation}

WKB theory, like ray theory, provides an approximate solution to the wave equation in the high frequency regime; however, the WKB method takes frequency information into account in the amplitude function as well as in the phase, and it accounts for frequency dependent effects such as diffraction which are ignored in standard ray theory. In general, the WKB method is a means for finding an approximate solution to a linear ordinary differential equation in which the highest order derivative is multiplied by a small parameter [5] and its use is not limited to underwater acoustics. In the problem at hand, the differential equation of interest is the equation for the depth dependent Green's function (equation (2.18))

$$
\frac{d^{2} \tilde{p}\left(k_{r}, z, z_{0}\right)}{d^{2} z}+k_{0}^{2}\left[n^{2}(z)-k_{r}^{2} / k_{0}^{2}\right] \bar{p}\left(k_{r}, z, z_{0}\right)=0
$$


where $k_{0}=\omega / c_{0}, c_{0}$ is the sound speed minimum, and $n(z)$ is the index of refraction. For later convenience we make the following change in variables

$$
\kappa(z)=n^{2}(z), \quad \xi=k_{r}^{2} / k_{0}^{2}
$$

and

$$
Q^{2}(z)=\kappa(z)-\xi
$$

Equation (2.3) then becomes

$$
\frac{d^{2} \tilde{p}\left(k_{r}, z, z_{0}\right)}{d^{2}}+k_{0}^{2} Q^{2}(z) \tilde{p}\left(k_{r}, z, z_{0}\right)=0
$$

This equation is solved by expanding $\tilde{p}$ as [22]

$$
\tilde{p}\left(k_{r}, z, z_{0}\right)=\exp \left[k_{0} \int_{z_{0}}^{z} d z \sum_{\nu=0}^{\infty} y_{\nu}(z) k_{0}^{-\nu}\right]
$$

where the lower limit $z_{0}$ is some conveniently chosen constant depth. Substituting this expansion into equation (2.44) and setting the coefficients of successive powers of $k_{0}$ equal to zero, we obtain

$$
\begin{aligned}
y_{0}(z) & = \pm i Q(z) \\
\frac{d y_{\nu-1}}{d z} & =-\sum_{\mu=0}^{\nu} y_{\mu} y_{\nu-\mu} ; \nu=1,2,3, \ldots
\end{aligned}
$$

from which the series in the exponent of equation (2.45) may be determined. This series will, in general, be asymptotic and not convergent. Retaining the first three terms and using a convenient normalization gives the second order WKB approximation

$$
\tilde{p}\left(k_{r}, z, z_{0}\right)=\left(k_{0} Q(z)\right)^{-1 / 2} \exp \left[ \pm \int_{z_{0}}^{z}\left(1+\frac{1}{2 \epsilon_{0}}\right) k_{0} Q(z) d z\right]
$$

where

$$
\epsilon_{0}=\left(Q(z) k_{0}\right)^{-3 / 2} \frac{d^{2}}{d z^{2}}\left(k_{0} Q(z)\right)^{-1 / 2}
$$

The steps leading to equation (2.48) are justified if

$$
\left|y_{2} k_{0}^{-2}\right| \ll\left|y_{1} k_{0}^{-1}\right| \ll\left|y_{0}\right|
$$

or

$$
\left|\epsilon_{0}\right| \ll\left|\frac{d}{d z}\left(k_{0} Q(z)\right)^{-1}\right| \ll 2
$$


If

$$
\left|\int_{z_{0}}^{z} k_{0} \epsilon_{0} Q(z) d z\right| \ll 2
$$

then we can make the physical optics approximation [5] of retaining only the first two terms, and the two linearly independent solutions of equation (2.3) are

$$
\tilde{p}\left(k_{r}, z, z_{0}\right)=k_{0}^{-1 / 2}(\kappa(z)-\xi)^{-1 / 4} \exp \left[ \pm i k_{0} \int_{z_{0}}^{z}(\kappa(z)-\xi)^{1 / 2} d z\right]
$$

The WKB method has provided an approximate solution to equation (2.18) which takes into account sound speed profile and frequency information, and which allows for frequency dependent effects such as diffraction. These WKB modal solutions can then be used in the modal sum (equation (2.24)) to approximate the full wave solution. It is well known that the WKB solution fails at the turning points i.e. the points at which $Q(z)=(\kappa(z)-\xi)=0$. As noted earlier, the behaviour of the solution differs on the two sides of the turning point with oscillatory behaviour above the turning point and an exponentially decaying behaviour below the turning point. There is an extensive literature concerning the problem of connecting the solutions through the turning point (see [5]); however, we are only interested in the phase memory of the solution i.e. the integral in the exponential for the region above the turning point, and not in generating approximations to the sound field. 


\section{Chapter 3}

\section{The Inversion}

We approach the inversion by first grouping modes according to the boundary interactions they undergo and the number of turning points. Based on these factors the WKB phase integral is evaluated for each type of mode resulting in an expression which depends only on the mode number in the case of modes which do not interact with the bottom, and on mode number and the phase of the bottom reflection coefficient for bottom interacting modes. Treating these expressions as functions of the variable $\xi$, we proceed, for each inversion method, to obtain an Abel integral equation which enables us to extract a relationship between the index of refraction and either the depth (inversion method 1) or excursion (inversion method 2). Both inversion methods are applied to three prototypal sound speed profiles to demonstrate their performance under ideal conditions. The performance of the inversions is then tested using one profile (the $n^{2}(z)$ profile ) with random errors added to the eigenvalues.

\subsection{Evaluating the Phase Integral}

There are four basic types of normal modes depending on the region where the normal modes are concentrated i.e. the region where $\sqrt{\kappa-\xi}$ is real [7] (sound speed profiles with multiple sound ducts are excluded from this discussion):

1. $0<z<z_{t_{\text {lower }}}$. The region is bounded above by the pressure release surface and below by a turning point. 
2. $z_{t_{\text {upper }}}<z<z_{t_{\text {lower }}}$. The region is bounded above by a turning point at $z_{t_{\text {upper }}}$ and below by a turning point at $z_{t_{\text {lower }}}$. On the ocean surface and bottom, the sound field is exponentially small. This case would include, for example, SOFAR propagation.

3. $0<z<H$. The region is bounded above by the ocean surface and below by the ocean bottom.

4. $z_{t_{\text {upper }}}<z<H$. The region is bounded above by a turning point and below by the ocean bottom.

Because we are interested in the properties of the sediments as well as the water column, we include in the categories having lower turning points, those instances in which the sound is not totally reflected at the interface, but turns within the sediment layers.

For convenience, let $z_{t_{1}}=z_{t_{l o w e r}}, z_{t_{2}}=z_{t_{\text {upper }}}$, and $\gamma=(\kappa-\xi)^{1 / 2}$. The WKB solution to the depth equation for the transform variable $\tilde{p}(z)$ is

$$
\begin{gathered}
\bar{p}(z)=\frac{1}{\sqrt{\gamma}}\left[C_{1} \exp \left(i k_{0} \int_{z}^{z_{t_{1}}} \gamma d z\right)+C_{2} \exp \left(-i k_{0} \int_{z}^{z_{t_{1}}} \gamma d z\right)\right] \\
z_{t_{2}}<z<z_{t_{1}} \\
\tilde{p}(z)=\frac{1}{\sqrt{|\gamma|}} C_{3} \exp \left(-k_{0} \int_{z_{t_{1}}}^{z}|\gamma| d z\right) ; \quad z_{t_{1}}<z .
\end{gathered}
$$

The two terms in equation (3.1) represent upgoing and downgoing waves in the sound channel while equation (3.2) represents an exponentially attenuated wave below the turning depth. The constants are related by [8]

$$
C_{1}=C_{3} \exp (i \pi / 4) ; \quad C_{2}=C_{3} \exp (-i \pi / 4)
$$

with the $\pi / 4$ factor accounting for the phase associated with turning at the turning depth. Our interest is above the turning depth where the solution is oscillatory, and that solution can be written

$$
\tilde{p}(z)=\frac{2 C_{3}}{\sqrt{\gamma}} \cos \left(k_{0} \int_{z}^{z_{t_{1}}} \gamma d z-\pi / 4\right)
$$

from which the phase integral will be evaluated using the boundary conditions. We are not interested in the value of the constants $C_{3}$, and will deal only with the phase integral. 
For the first mode type (case 1), the pressure release condition at the ocean surface requires that $\tilde{p}(0)=0$. Setting the cosine term equal to zero requires

$$
k_{0} \int_{0}^{z_{t_{1}}} \gamma_{n} d z=(n-1 / 4) \pi ; \quad n=1,2,3, \ldots
$$

For the modes with two turning points (case 2), we assume that the turning points are far enough away from the boundaries that interactions with the boundaries are not of concern. Expanding the cosine into the sum of exponentials

$$
\begin{gathered}
\cos \left(k_{0} \int_{z}^{z_{t_{1}}} \gamma_{n} d z-i \pi / 4\right)=\frac{1}{2} \exp \left(i k_{0} \int_{z}^{z_{t_{1}}} \gamma_{n} d z-i \pi / 4\right) \\
+\frac{1}{2} \exp \left(-i k_{0} \int_{z}^{z_{t_{1}}} \gamma_{n} d z+i \pi / 4\right) .
\end{gathered}
$$

The first term is a wave propagating in the positive $z$ direction (down), while the second term is a wave propagating in the negative $z$ direction (up) i.e. a wave which was reflected at the upper turning point. At the turning depth, the wave is reflected with a $\pi / 2$ phase change. Taking the ratio of the two terms and setting the ratio equal to $\exp [i \pi(2 m+1)]$ gives

$$
k_{0} \int_{z_{t_{2}}}^{z_{t_{1}}} \gamma_{n} d z=(n-1 / 2) \pi ; \quad n=1,2,3, \ldots
$$

with $m=(n-1)$ used to provide indices starting at 1 .

For the case 3 we consider a mode interacting with the pressure- release surface above and totally reflected at the bottom. Expanding the field into up and downgoing waves again gives

$$
\tilde{p}(z)=A \exp \left(i k_{0} \int_{0}^{z} \gamma_{n} d z\right)+B \exp \left(-i k_{0} \int_{0}^{z} \gamma_{n} d z\right)
$$

Evaluating the terms at the two boundaries in terms of the surface and bottom reflection coefficients

$$
R_{s}=\frac{\tilde{p}(0) \downarrow}{\tilde{p}(0) \uparrow}=\frac{A}{B}
$$

and

$$
R_{b}=\frac{\tilde{p}(H) \uparrow}{\tilde{p}(H) \downarrow}=\frac{B}{A} \exp \left(-2 i \int_{0}^{H} \gamma_{n} d z\right) .
$$

Eliminating the ratio $A / B$ leaves

$$
R_{b} R_{s} \exp \left(2 i \int_{0}^{H} \gamma_{n} d z\right)=1
$$


Letting $R_{s}=\exp \left(i 2 \Phi_{s}\right)$ and $R_{b}=\exp \left(i 2 \Phi_{b}\right)$, gives

$$
\Phi_{s}+\Phi_{b}+\int_{0}^{H} \gamma_{n} d z=(n-1) \pi ; \quad n=1,2,3, \ldots
$$

For a pressure release surface $\Phi_{s}=-\pi / 2$ and

$$
\int_{0}^{H} \gamma_{n} d z=(n-1 / 2) \pi-\Phi_{b}
$$

where $\Phi_{b}$ is the phase of the reflection coefficient for total internal reflection at the angle of incidence for the given mode. In the case of the Pekeris waveguide ( an isovelocity water column over an isovelocity bottom half-space) this is [11]

$$
\begin{aligned}
\Phi_{b} & =\arctan \frac{b_{1} \rho_{0} c_{0}}{\rho_{1} c_{1} \cos \theta_{0}} \\
b_{1} & =\left[\left(\frac{c_{1}}{c_{0}}\right)^{2} \sin ^{2} \theta_{0}-1\right]^{1 / 2}
\end{aligned}
$$

where the $\rho$ are the densities, $c$ are the sound speeds, and $\theta_{0}$ is the angle of incidence in the upper medium. The subscript o refers to the upper medium, and the subscript 1 refers to the lower medium.

In case 4 the wave reflected at the upper turning depth lags in phase by $\pi / 2$ behind the incident wave [7], and $\Phi_{s}=-\pi / 4$ giving for the phase integral

$$
k_{0} \int_{z_{\mathrm{t}}}^{H} \gamma_{n} d z=(n-3 / 4) \pi-\Phi_{b} ; \quad n=1,2,3, \ldots
$$

\subsection{Derivation of the Inversion Relations}

We designate the WKB phase integral divided by the wavenumber $k_{0}$ as $F(\xi)$. The variable $\xi$ is physically equal to $\sin ^{2} \theta$ where $\theta$ is the angle of incidence of the ray corresponding to the mode having the eigenvalue $k_{0} \sqrt{\xi}$. Viewing the modes as a consequence of constructive interference of rays, we treat $\xi$ as a continuous variable with the measured values of the horizontal wavenumber and the WKB phase integral defining values of the function $F(\xi)$ at selected points. An Abel integral equation which can be used to relate depth to the index of refraction (or sound speed depending on the normalization used) may be obtained by two methods. The first approach is applicable to sound speed profiles which are monotonically increasing with depth or symmetric with respect to the sound speed minimum. The second 
approach allows determination only of the distance between points on the two branches of the sound speed profile having the same sound speed when the profile is asymmetric. If some of the modes for the asymmetric profile have two turning points and some have one turning point, then for sound speed range for which there are two turning points the result will be as described, but for the sound speed range with one turning point, the result will be the sound speed profile. For a sound speed profile which is monotonically increasing with depth or is symmetric about the sound speed minimum, the second method, like the first, provides depth as a function of index of refraction. We will refer to the first method which is applicable to monotonic and symmetric profiles as inversion method 1 , and we will refer to the second, more general, method as inversion method 2 .

\subsubsection{Derivation of inversion method 1}

Considering the case of a profile which is monotonically increasing with depth, the phase integral of the WKB solution to the wave equation (assuming constant density) is written as

$$
\int_{0}^{z_{t}}\left[n^{2}(z)-\left(k_{r_{n}} / k_{0}\right)^{2}\right]^{1 / 2} d z=\frac{(n+1 / 2) \pi}{k_{0}}
$$

Here $k_{0}$ is $\omega / c_{0}, c_{0}$ is the value of the sound speed at the surface, and $k_{r_{n}}$ is the horizontal wavenumber for the $n$th mode. The equation has been normalized by dividing through by $k_{0}$, and the quantity which will ultimately result from the inversion is the index of refraction $n(z)$. Here $z_{t}$ is used to denote the single turning depth. If the sound speed profile is symmetric, the phase integral is taken between upper and lower turning points, and the symmetry of the problem allows the integral to be split into two equal pieces with the resulting factor of two taken to the right-hand side of the equation.

With the change of variables from the previous chapter

$$
\kappa(z)=n^{2}(z) ; \quad \xi=\left(k_{r_{n}} / k_{0}\right)^{2}
$$

equation (3.16) is written as

$$
\int_{0}^{z_{t}}[\kappa(z)-\xi]^{1 / 2} d z=F(\xi)
$$

Following the treatment of Vassell [43], this can be transformed into an Abel integral equation 
by differentiating with respect to $\xi$ giving

$$
\frac{d F(\xi)}{d \xi}=H(\xi)=-\frac{1}{2} \int_{0}^{z_{t}} \frac{d z}{[\kappa(z)-\xi]^{1 / 2}}
$$

The independent variable $z$ is eliminated in favor of the dependent variable $\kappa$ by letting

$$
z=-\int_{\kappa}^{1} G(\kappa) d \kappa
$$

where $G(\kappa)$ is an unknown function, and in fact we will not have any reason to determine $G(\kappa)$ explicitly.

Since $\kappa(z)$ is the index of refraction squared, at the reference depth $\kappa(0)=1$, and at the turning depth $\kappa\left(z_{t}\right)=\xi$. Equation (3.18) becomes

$$
H(\xi)=\frac{1}{2} \int_{\xi}^{1} \frac{G(\kappa) d \kappa}{[\kappa-\xi]^{1 / 2}} .
$$

which is an Abel integral equation and can be inverted [28] to give

$$
G(\kappa)=-\frac{2}{\pi} \frac{d}{d \kappa} \int_{\kappa}^{1} \frac{H(\xi) d \xi}{[\xi-\kappa]^{1 / 2}} .
$$

Using equation (3.19) and integrating gives the relation

$$
z=-\frac{2}{\pi} \int_{\kappa}^{1} \frac{H(\xi) d \xi}{[\xi-\kappa]^{1 / 2}}
$$

which can be used to calculate $\kappa(z)$ from the $H(\xi)$ obtained by differentiating the function $F(\xi)$ defined by the measured horizontal wavenumbers and associated values of the WKB phase integral. The value of the sound speed at one depth is now used to convert the index of refraction to a sound speed profile.

\subsubsection{Derivation of inversion method 2}

In the case of an asymmetric sound channel with two turning points, we are able to obtain a relation for the distance between the points on the two branches of the sound speed profile having the same sound speed. The information contained in the phase integral is insufficient to obtain the depths separately. An analagous problem in quantum mechanics is the determination of a potential from bound state information and the technique we use is referred 
to as the JWKB semi- classical approximation in the quantum mechanical context [10],[44]. Define the inclusion as

$$
I(\xi)=\int_{z_{2}}^{z_{1}}(\kappa-\xi) d z
$$

and the excursion as

$$
X(\xi)=\int_{z_{2}}^{z_{1}} d z=z_{1}-z_{2}
$$

It is the excursion which we will be able to obtain from measurements of the horizontal wavenumbers and associated values of the phase integral. For a monotonic profile, the second turning point is at the surface $(z=0)$, and the excursion will give the index of refraction versus depth directly, and in asymmetric channels the surface is the upper turning point for modes interacting with the surface so that the excursion will be the sound velocity profile for a portion of the profile.

Define the adjoint fractional integral as [39]

$$
{ }_{x} W_{b}^{-\nu} f(x)=\frac{1}{\Gamma(\nu)} \int_{x}^{b}(\zeta-x)^{\nu-1} f(\zeta) d \zeta ; \quad \text { Re } \nu>0 .
$$

Applying this to both sides of the WKB phase integral equation with $\nu=-1 / 2$ gives

$$
\int_{\xi}^{\xi_{\max }}\left(\xi^{\prime}-\xi\right)^{1 / 2}\left[\int_{z_{1}}^{z_{2}}\left(\kappa-\xi^{\prime}\right)^{1 / 2} d z\right] d \xi^{\prime}=\int_{\xi}^{\xi_{\max }}\left(\xi^{\prime}-\xi\right)^{-1 / 2} F\left(\xi^{\prime}\right) d \xi^{\prime}
$$

Figure (3-1) shows the region over which the square of the index of refraction is being integrated. Interchanging the order of integration, it is apparent that the integral over $\xi^{\prime}$ will be zero for $\xi$ greater than $\kappa$. This leaves

$$
\int_{z_{2}}^{z_{1}} d z\left[\int_{\xi}^{\kappa}\left(\frac{\kappa-\xi^{\prime}}{\xi^{\prime}-\xi}\right)^{1 / 2} d \xi^{\prime}\right]=\int_{\xi}^{\kappa} \frac{F\left(\xi^{\prime}\right)}{\left(\xi^{\prime}-\xi\right)^{1 / 2}} d \xi^{\prime}
$$

which reduces to

$$
I(\xi)=\int_{z_{2}}^{z_{1}}(\kappa-\xi) d z=\frac{2}{\pi} \int_{\xi}^{\kappa} \frac{F\left(\xi^{\prime}\right)}{\left(\xi^{\prime}-\xi\right)^{1 / 2}} d \xi^{\prime} .
$$

Taking the derivative of both sides of this equation with respect to $\xi$ results in an expression for the excursion

$$
X(\xi)=\int_{z_{2}}^{z_{1}} d z=-\frac{\partial}{\partial \xi}\left[\frac{2}{\pi} \int_{\xi}^{\kappa} \frac{F\left(\xi^{\prime}\right)}{\left(\xi^{\prime}-\xi\right)^{1 / 2}} d \xi^{\prime}\right] .
$$

Thus from the values of $F(\xi)$, we determine the inclusion for the sound speed profile, and use the inclusion to find the excursion. 


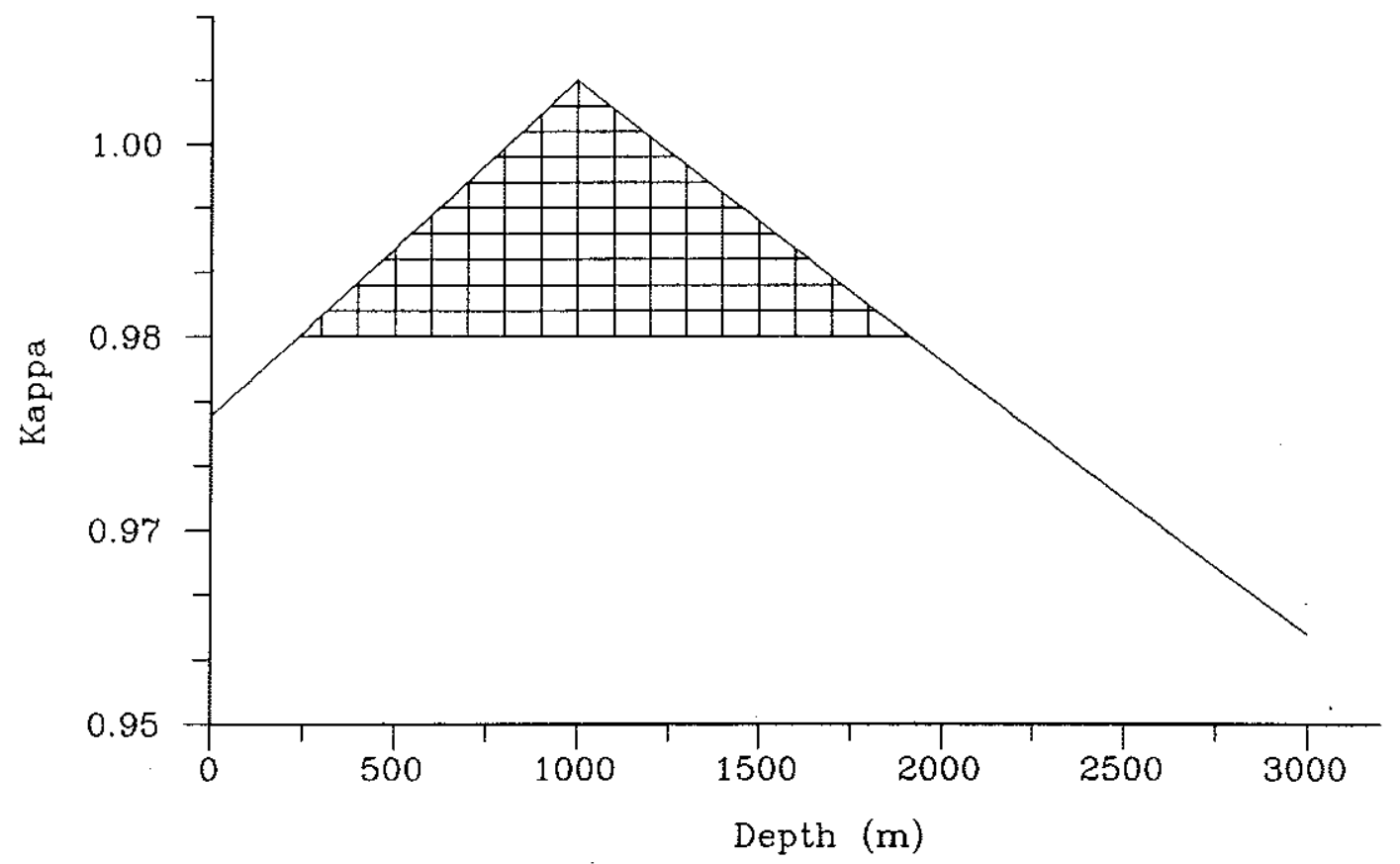

Figure 3-1: The integration over $\xi^{\prime}$ is carried out over the cross-hatched region extending from $\xi$ to the curve $\kappa(z)$.

\subsubsection{Examples of analytically invertible profiles}

The $n^{2}(z)$ linear profile and the parabolic profile are two examples of sound speed profiles which are analytically invertible with these techniques, and which have well known expressions for the eigenvalues. These profiles will serve as test cases for checking the accuracy of the intermediate steps in the numerical inversions.

\section{The $n^{2}(z)$ linear profile}

With the square of the index of refraction given by

$$
\kappa(z)=n^{2}(z)=1-a z
$$

the WKB phase integral can be evaluated directly

$$
F(\xi)=\int_{0}^{z_{t}}[(1-a z)-\xi]^{1 / 2} d z=\frac{2}{3 a}(1-\xi)^{3 / 2} .
$$


Using the first inversion method we take the derivative with respect to $\xi$ giving

$$
H(\xi)=\frac{-1}{a}(1-\xi)^{1 / 2}
$$

The integral expression for depth in terms of the index of refraction is

$$
z=\frac{2}{a \pi} \int_{\kappa}^{1}\left(\frac{1-\xi}{\xi-\kappa}\right)^{1 / 2} d \xi
$$

which upon integrating and solving for $\kappa$ returns the original expression for the square of the index of refraction.

\section{The parabolic profile}

For the parabolic profile the square of the index of refraction is given by

$$
\kappa(z)=n^{2}(z)=1-\alpha^{2} z^{2}
$$

and the inclusion is

$$
I(\xi)=\int_{z_{t_{2}}}^{z_{t_{1}}}\left[\left(1-\alpha^{2} z^{2}\right)-\xi\right] d z=\frac{4}{3 \alpha}(1-\xi)^{3 / 2} .
$$

Taking the derivative with respect to $\xi$ gives the excursion

$$
-\frac{\partial}{\partial \xi}=X(\xi)=\frac{2}{\alpha}(1-\xi)^{1 / 2}
$$

The depth of the turning points is given by the condition

$$
\left[\left(1-\alpha^{2} z^{2}\right)-\xi\right]=0 \text { or } z= \pm(1-\xi)^{1 / 2} / \alpha
$$

and the excursion is twice this as expected.

\subsection{Implementation}

In order to determine the sound speed with these methods, we require a set of measurements of the horizontal wavenumber in the waveguide and the sound speed for at least one depth ( this last permits conversion of the index of refraction profile to a sound speed profile). For inversion method 2, we assume that the sound speed at the surface and both the depth an value of the minimum sound speed in the profile are known. From the calculations of the WKB 
phase integral values for the various cases, it is clear that the mode numbers of the measured eigenvalues must be correctly identified, and that the boundary interactions experienced by each mode must be correctly identified. From the eigenvalues and associated WKB phase integral values, the variables $\xi=\left(k_{r_{n}} / k_{0}\right)^{2}$ and $F(\xi)$ are computed. One additional point, corresponding to horizontally propagating energy, is added at $\xi=1$ and $F(\xi=0)=0$.

The first step in either inversion method is to fit the data using either a least squares basis spline or a cubic smoothing spline. Either of these two spline methods allows for noise in the data. The spline routines essentially provide a piecewise polynomial that provides a best fit to the data in a sense which depends on the type of spline routine used. The use of splines is convenient in that the integrations and differentiations required for the inversion are easily performed once the coefficients for the spline representaion have been computed. All the computer routines used for fitting the data with the spline, differentiation, and integration are in the IMSL, Inc. MATH/LIBRARY [1] .

Before discussing the differences in the two spline fitting methods, it is worthwhile to define the basis spline which first requires a definition of the divided difference (for a complete discussion of splines see reference [14]). For a function $g$ which is given at the points $x_{i}, \ldots, x_{i+k}$, the $k t h$ divided difference of $g$ at the points $x_{i}, \ldots, x_{i+1}$ is the leading coefficient of the polynomial of order $k+1$ which agrees with $g$ at the points $x_{i}, \ldots, x_{i+k}$; it is denoted by $\left[x_{i}, \ldots, x_{i+k}\right] g$. The agreement between the two functions referred to here means that if a point occurs $m$ times in the sequence $\boldsymbol{x}$, then the two functions and their derivatives agree $m-$ fold in that the two functions and the derivatives up to the $(m-1)$ th derivative are equal. For a sequence $\left(x_{0}, x_{1}, \ldots, x_{N}\right)$, if we let

$$
\Psi_{N}(x)=\left(x-x_{0}\right) \ldots\left(x-x_{N}\right)
$$

and

$$
\Psi_{N}^{\prime}\left(x_{i}\right)=\left(x_{i}-x_{0}\right) \ldots\left(x_{i}-x_{i-1}\right)\left(x_{i}-x_{i+1}\right) \ldots\left(x_{i}-x_{N}\right)
$$

(i.e. the $\left(x-x_{i}\right)$ factor is removed before evaluating the product at the point $\left.x_{i}\right)$, then the divided difference is [4]

$$
\left[x_{0}, \ldots, x_{N}\right] g=\sum_{j=0}^{N} \frac{g\left(x_{j}\right)}{\Psi_{N}^{\prime}\left(x_{j}\right)}
$$


The basis spline or B-spline is defined as follows [14]: for a nondecreasing sequence $t \equiv\left(t_{i}\right)$, the $i t h$ (normalized) B-spline of order $k$ for the knot sequence $t$ is denoted by $B_{i, k, t}$, and is defined by the rule

$$
b_{i, k, t} \equiv\left(t_{i+k}-t_{i}\right)\left[t_{i}, \ldots, t_{i+k}\right](\cdot-x)_{+}^{k+1} .
$$

The truncation function $(x-t)_{+}$is defined as $\max \{0, x-t\}$ and the notation is used to indicate that $x$ is fixed and $(t-x)_{+}^{k-1}$ is to be considered as a function of $t$ alone. Note that $B_{i, k, t}(x)$ is 0 for $x \notin\left[t_{i}, t_{i+k}\right]$. A spline function of order $k$ with knot sequence $t$ is any linear combination of B-splines of order $k$ for the knot sequence $t$.

The least squares spline routine calculates a weighted discrete $L_{2}$ approximation to the given data $\left(x_{i}, f_{i}\right)$ for $i=1,2, \ldots, N$, (it finds the coefficients $a_{j}$ ) to minimize the weighted square error between the data and the spline i.e.

$$
\sum_{i=1}^{N}\left|f_{i}-\sum_{j=1}^{m} a_{j} B_{j}\left(x_{i}\right)\right|^{2} w_{i} .
$$

The number of data points is $N, B_{j}$ is the $j$ th spline of order $k, m$ is the number of coefficients (the number of B-splines making up the polynomial representation), and $w_{i}$ are the data weights. The order $k$ is the order of the polynomial pieces ( a polynomial of order $k$ is a polynomial of degree $k-1$; for a cubic polynomial $k=4$ ). In general, the weights for our problem have been selected such that the measured data are equally weighted, and the added point at $(1,0)$ is very heavily weighted compared to the measurements. These weights can be tailored to fit the confidence in the measurements.

The smoothing spline is a natural cubic spline approximation with knots at the data abscissas where the term 'natural' refers to the end condition imposed. In addition to the constraints imposed by the required agreement between the function and the spline at the knots, some conditions or constraints must be given at the endpoints of the interval on which the spline is being calculated. In the case of the natural spline of order $k=2$ and $N$ data points, the condition specified is

$$
f^{(j)}\left(x_{1}\right)=f^{(j)}\left(x_{N}\right)=0 ; \quad j=m, \ldots, k-2
$$

which in the case of a cubic spline $(m=2)$ becomes

$$
f^{(2)}\left(x_{1}\right)=f^{(2)}\left(x_{N}\right)=0 .
$$


In our problem, the data error variance is unknown, and the required amount of smoothing is determined in the IMSL smoothing spline routine by using the generalized cross validation method of Craven and Wahba [13]. Modelling the data as

$$
y(t)=g(t)+\epsilon(t) ; \quad t \in[0,1]
$$

the problem is to construct $g(t)$ from the discrete data points $y_{i}$. The function $g(t)$ is assumed to be in $W_{2}^{(m)}$, where

$$
W_{2}^{(m)}=\left\{g: g^{\nu} \text { abs. cont., } \nu=0,1, \ldots, m-1, g^{(m)} \in L_{2}[0,1]\right\} .
$$

The estimate of $g$ is $g_{n, \lambda}$, the solution to the problem: Find $f \in W_{2}^{(m)}$ to minimize

$$
\frac{1}{n} \sum_{j=1}^{n}\left(f\left(t_{j}\right)-y_{j}\right)^{2}+\lambda \int_{0}^{1}\left(f_{(m)}(u)\right)^{2} d u .
$$

The solution $g_{n, \lambda}$ is the smoothing spline of order $2 m-1$ [38] with the parameter $\lambda$ controlling the tradeoff between the smoothness of the solution measured by

$$
\int_{0}^{1}\left[f^{(m)}(u)\right]^{2} d u
$$

and the infidelity to the data measured by

$$
\frac{1}{n} \sum_{j=1}^{n}\left(f\left(t_{j}\right)-y_{j}\right)^{2}
$$

The generalized cross-validation estimate for the minimization of the average square error is defined by [13]

$$
V(\lambda)=\frac{1}{n}\|(I-A(\lambda)) y\|^{2} /\left[\frac{1}{n} \operatorname{Trace}(I-A(\lambda))\right]^{2},
$$

where $y=\left(y_{1}, \ldots, y_{n}\right)^{t}$ and $A(\lambda)$ is the $n \times n$ matrix satisfying

$$
\left(g_{n, \lambda}\left(t_{1}\right), \ldots, g_{n, \lambda}\left(t_{n}\right)\right)^{t}=A(\lambda) y .
$$

Once the initial spline fit to the data has been done using either of the two methods, the paths of the two inversion methods diverge. The important points related to splines for this work are: (1) the spline routines fit piecewise polynomials to the data (the polynomial pieces are generally cubic, although the least squares routine allows the order of the polynomial pieces to be specified); (2) the spline routines used allow for the presence of noise in the data; and (3) the integrations and differentiations are based on the polynomial coefficients from the spline fit to the data. 


\subsubsection{Implementation of inversion method 1}

The function $H(\xi)$ is generated by taking the derivative of the spline representation of $F(\xi)$ at the data points $\xi_{i}$ of the input data. A spline is then fitted to $H(\xi)$. Since noise in the original data may be amplified in the processs of computing the derivatives, a smoothing or least squares spline is also used at this stage of the computations. The integral that must now be evaluated is

$$
z=-\frac{2}{\pi} \int_{\kappa}^{1} \frac{H(\xi) d \xi}{(\xi-\kappa)^{1 / 2}}
$$

which has a singularity at $\kappa$. To avoid problems with the singularity during integration, the change of variables $([4],[43]) u^{2}=(\xi-\kappa)$ is made and the integral to be evaluated becomes

$$
z=-\frac{4}{\pi} \int_{0}^{(1-\kappa)^{1 / 2}} H\left(\kappa+u^{2}\right) d u
$$

This is evaluated at points over the range of $\kappa$ (which coincides with the range of $\xi$ ) using the spline and integration routines. The one required measurement of $c(z)$ is then used to convert the points of $(z, n(z))$ to a sound speed profile $c(z)$.

\subsubsection{Implementation of inversion method 2}

The integral which must be evaluated to obtain the inclusion is

$$
I(\xi)=\frac{2}{\pi} \int_{\xi}^{\kappa} \frac{F\left(\xi^{\prime}\right)}{\left(\xi^{\prime}-\xi\right)^{1 / 2}} d \xi^{\prime}
$$

which is of the same form as equation (3.38), and the same change of variables is used to eliminate the singularity. The integration is again done using the same spline routines. The resulting points of the function $I(\xi)$ are fitted with a spline and differentiated with respect to $\xi$ to produce the desired result, $-X(\xi)$.

\subsection{Application of the Inversion Methods to the Analytic Profiles}

The two analy tically solvable profiles introduced earlier provide excellent examples with which to test the inversion methods since the results of the inversion calculations can be compared to known values during the intermediate stages of the computations. Expressions for the 
eigenvalues of these sound speed profiles are well known, and the eigenvalues can be computed without the use of a propagation modelling program. In the case of the $n^{2}(z)$ linear profile, we take the ocean as a half space with a pressure release surface, and use $a=2.28 \times 10^{-5} \mathrm{~m}^{-1}$ (this gives a sound speed gradient of $0.017 \mathrm{sec}^{-1}$ at a depth of 1000 meters corresponding to sound speed profile where pressure is the only factor affecting the sound speed i.e. an isovelocity, isosaline ocean). For the parabolic profile the ocean is treated as an infinite medium, and the coefficient $\alpha$ in the equation for $n^{2}(z)$ is $1.257 \times 10^{-4} \mathrm{~m}^{-2}$. In each case the 50 eigenvalues used have been calculated for a frequency of $220 \mathrm{~Hz}$ and a 1500 meter per second reference sound speed. Unless otherwise specified, all the calculations have been done using the least squares basis splines.

\subsubsection{Method 1 applied to the $n^{2}(z)$ linear profile}

The data points for the function $F(\xi)$ are created by dividing the input values of the horizontal wavenumber by $k_{0}=\omega / c_{0}$, and squaring the result to give $\xi$. The eigenvalues for this example were calculated using the expression [8]

$$
k_{r_{n}}^{2}=k_{0}^{2}-y_{n} / H^{2}
$$

where $y_{n}$ are the Airy function zeros, $H=\left(a k_{0}^{2}\right)^{-1 / 3}$ (equation(2.28)), and $a$ is the coefficient in the equation for the index of refraction. The asymptotic expressions [2] were used for the Airy function zeros; for the particular parameters used in thes examples this approximation did not introduce a significant error. The corresponding value of $F(\xi)$ is obtained by dividing the assumed value of the WKB phase integral by $k_{0}$. For this profile, all modes interact with the surface and have one turning point, and the WKB phase integral is given by equation

$$
\int_{0}^{z_{t}}(\kappa-\xi)^{1 / 2} d z=\pi(n-1 / 4) ; \quad n=1,2,3, \ldots
$$

The point at $(1,0)$ is added to the data set, and the spline coefficients calculated. Figure (3-2) shows the function $F(\xi)$ for this particular case. The background curve represents $F(\xi)$ computed using the analytic expression, and the discrete points are the values from the inversion calculations showing that there is good agreement between the actual value of the phase integral and the assumed value. Using the spline coefficients, the first derivative 


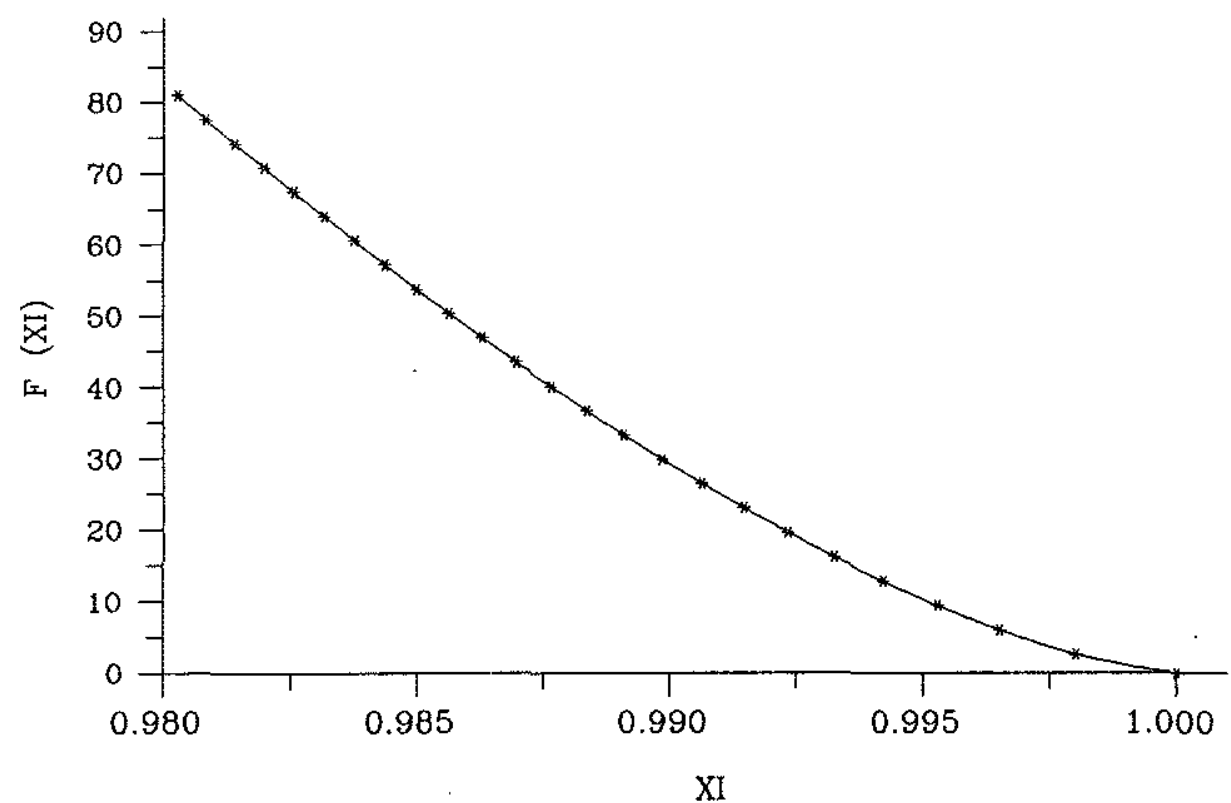

Figure 3-2: $F(\xi)$ for the $n^{2}(z)$ linear profile. The discrete points are the input data to the inversion program generated from the horizontal wavenumbers and the associated values of the WKB phase integral. The curve is $F(\xi)$ computed using the analytic expression.

of $F(\xi)$ with respect to $\xi$ is computed giving $H(\xi)$ as shown in figure (3-3). In calculating $H(\xi)$, a noticeable error occurs near $\xi=1.0$ due to the end conditions in the spline fit for $F(\xi)$ which do not coincide exactly to the behaviour of the function.

A spline is fitted to the function $H(\xi)$ represented by the the resulting discrete values of $H(\xi)$, and, using the change of variables $u^{2}=(\xi-\kappa)$, the integral

$$
z=-\frac{2}{\pi} \int_{0}^{(1-\kappa)^{1 / 2}} H\left(\kappa+u^{2}\right) d u
$$

is evaluated by varying $\kappa$ through the range for which input data is available. In essence, for each chosen value of $\kappa$, the depth at which a ray with the local propagation angle defined by $\kappa$ is found. As a matter of convenience the range of $\kappa$ or $\xi$ has been equally divided into the number of equal segments corresponding to the desired number of depth values to be computed. Figure (3-4) shows the results of the inversion for the $n^{2}(z)$ linear profile using 50 modes calculated at a frequency of $220 \mathrm{~Hz}$. The convention in presenting sound speed 


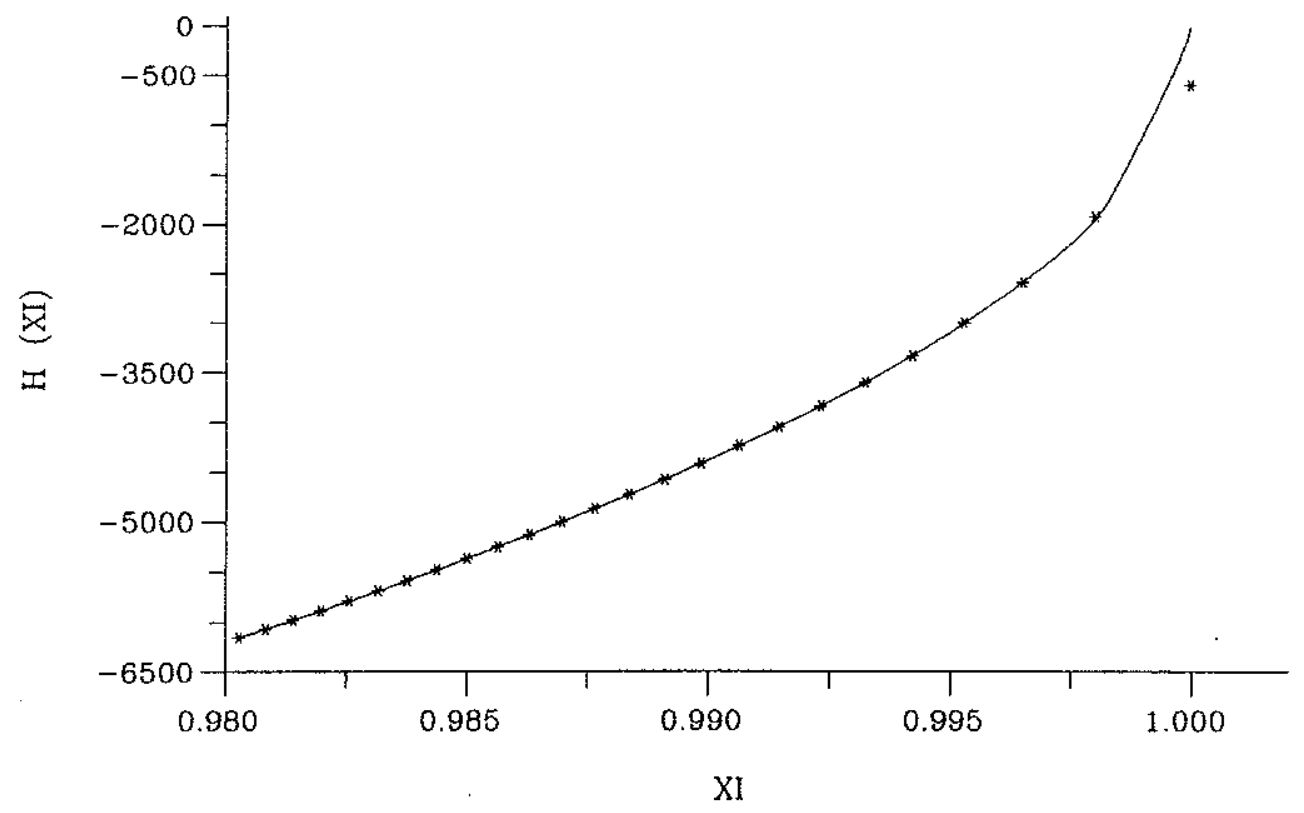

Figure 3-3: $H(\xi)$ for the $n^{2}(z)$ linear profile. The discrete points are the values of $H(\xi)$ computed by the inversion program. The curve is the function calculated using the analytic expression.

profiles will be to show inversion outputs as discrete points with the true profile plotted as a solid curve. The sound speed error as a function of depth for this case is shown in figure (3-5). Note that the largest errors occur near the surface where the spacing between the data points along the $\xi$ axis is greatest, and where the extrapolation from the measured data to the added point placed at $\xi=0$ is made. This region also corresponds to the region with the greatest error in the calculated values of $H(\xi)$.

\subsubsection{Method 2 applied to the $n^{2}(z)$ linear profile}

The initial data preparation is the same for both inversion methods since this is a monotonic profile. Once the spline has been computed for the function $F(\xi)$, the inclusion is computed from equation (3.40) using the change of variables $u^{2}=\xi^{\prime}-\xi$ to avoid problems with the 


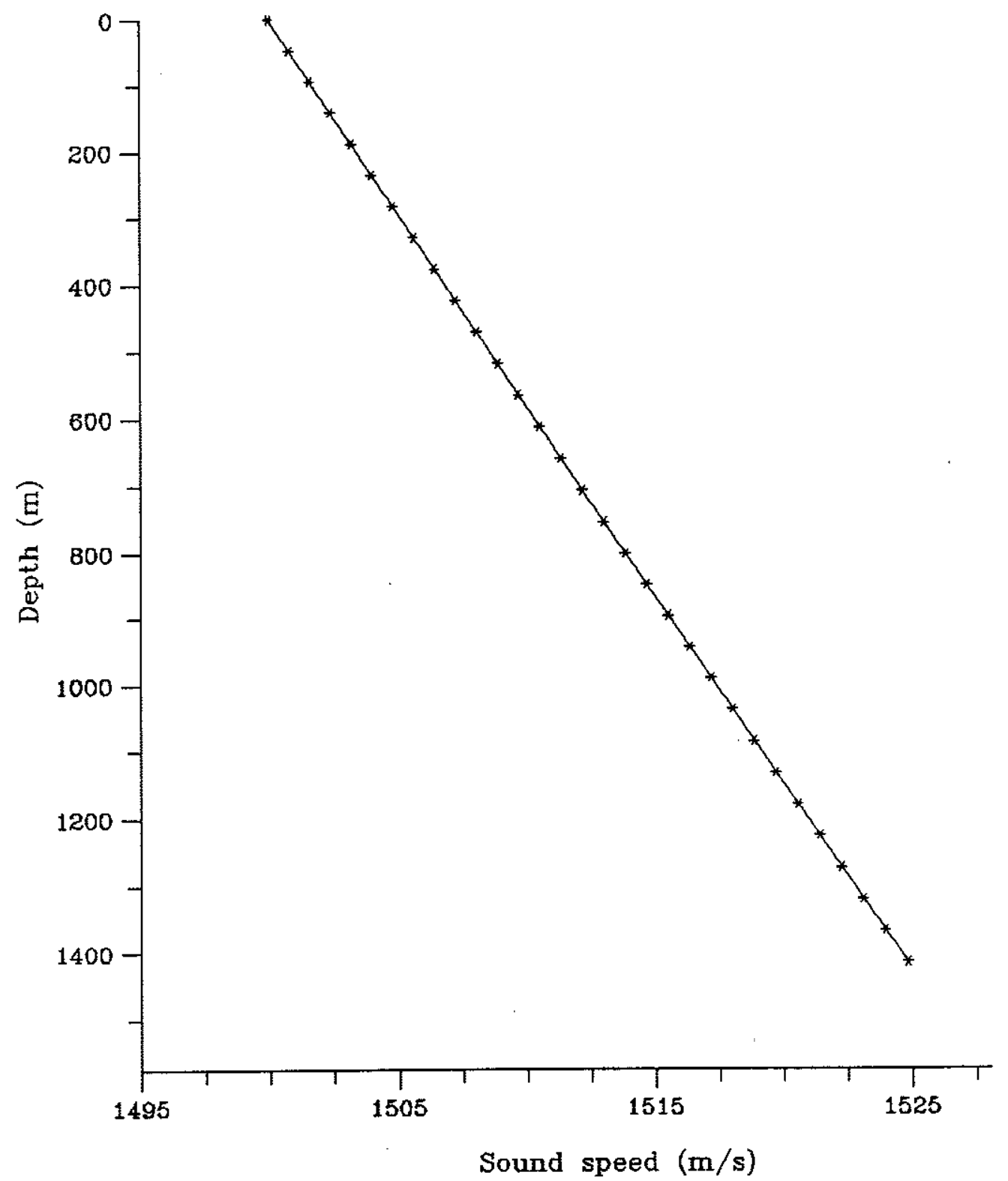

Figure 3-4: The sound speed profile for the $n^{2}(z)$ profile recovered using inversion method 1 . The discrete points are the output values from the inversion. 


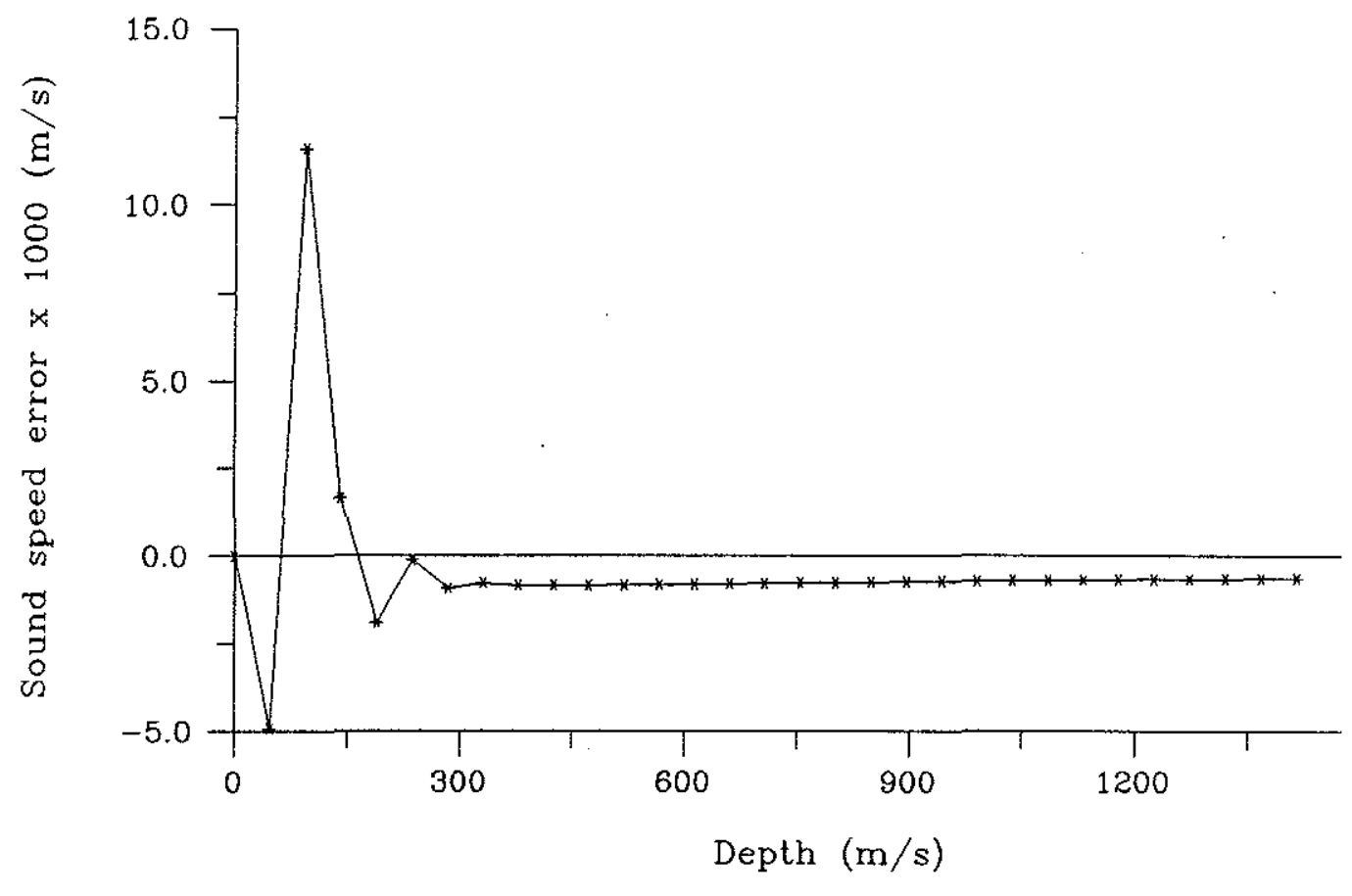

Figure 3-5: Sound speed error as a function of depth for the recovery of the $n^{2}(z)$ linear profile using inversion method 1. 
singularity at $\xi^{\prime}=\xi$. The integral to be evaluated becomes

$$
I(\xi)=\frac{4}{\pi} \int_{0}^{\sqrt{1-\xi}} F\left(u^{2}+\xi\right) d u
$$

After computing a spline fit to the points of $I(\xi)$ generated by the integration, we take minus the derivative of $I(\xi)$ with respect to $\xi$ to arrive at the excursion as shown in figure (3-6). The error, plotted as sound speed error as a function of depth, is shown in figure (3-7).

\subsubsection{Method 1 applied to the parabolic profile}

The values of $\xi$ for the given input data are calculated in the same way as for the previous case; however, the values of $F(\xi)$ are calculated using equation (3.6)

$$
k_{0} \int_{z_{t_{2}}}^{z_{t_{1}}}(\kappa-\xi)^{1 / 2} d z=\pi(n-1 / 2) ; \quad n=1,2,3, \ldots
$$

since there are two turning points and no boundary interactions for this profile. Because the turning depths are equidistant from the channel axis, $F(\xi)$ is divided by two, and the inversion proceeds as before. The resulting sound speed profile is for the region below the channel axis, and it is simply a matter of using the profile symmetry to generate the other half of the profile. Figure (3-8) shows the results of applying inversion method 1 to the parabolic profile, and figure (3-9) is plot of the inversion error presented as excursion error as a function of depth. Excursion error as a function of depth is a clearer way to evaluate the inversion results for two branch profiles recovered using method 2, and for consistency the errors for inversion method 1 are presented in the same manner. The excursion error in the case of a symmetric profile recovered using inversion method 1 is twice the error of the output for the single branch of the profile.

\subsubsection{Method 2 applied to the parabolic profile}

The parabolic profile involves modal interactions with two turning points, and inversion method 2 gives only excursion as a function of sound speed. Given prior knowledge that the profile is symmetric this may, if desired, be converted to a sound speed profile using symmetry as in inversion method 1 . Figure (3-10) is the excursion for the parabolic profile computed using inversion method 2, and figure (3-11) plots excursion error as a function of sound speed for this inversion. 


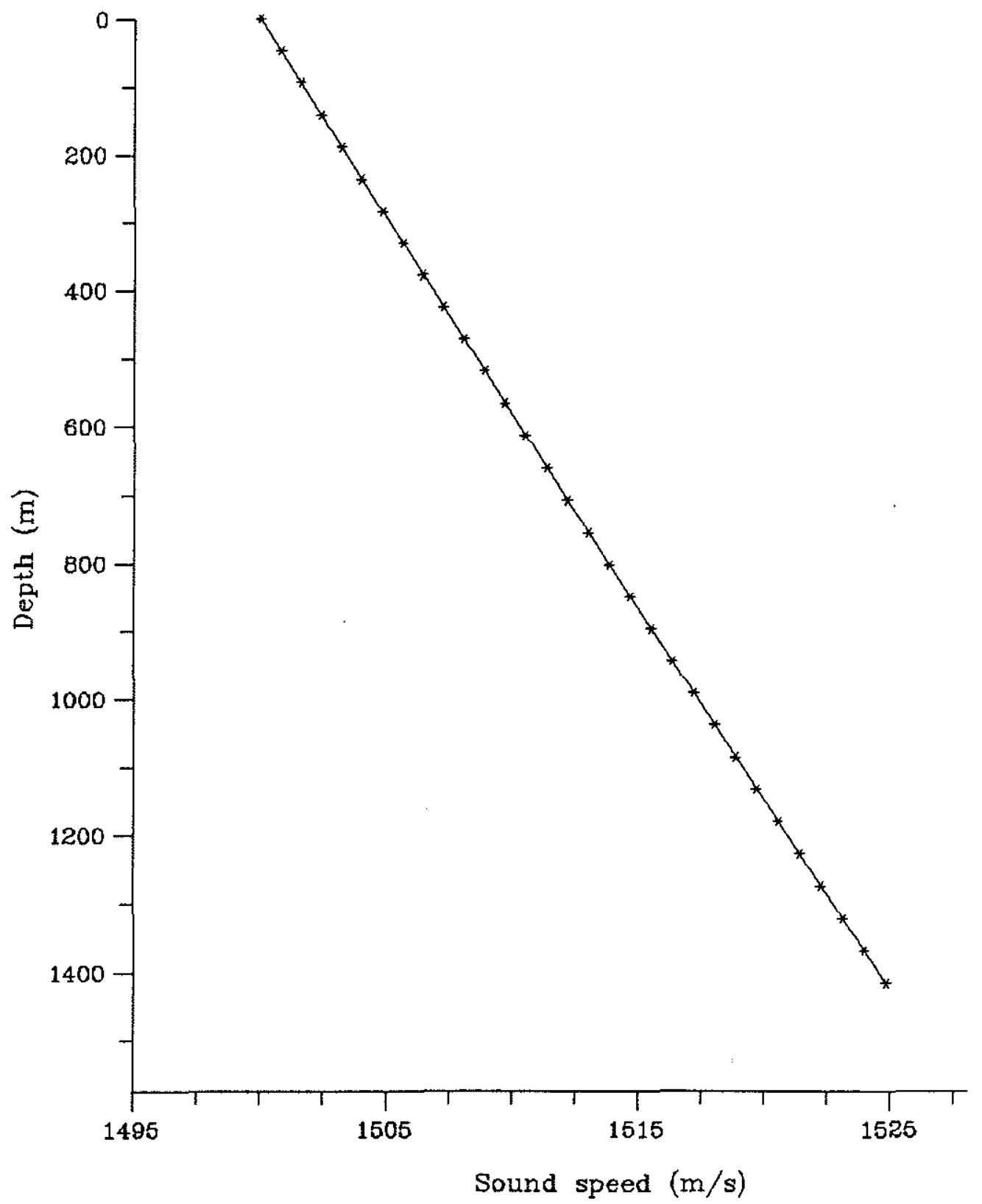

Figure 3-6: Excursion (depth) versus sound speed for the $n^{2}(z)$ linear profile calculated using inversion method 2. 


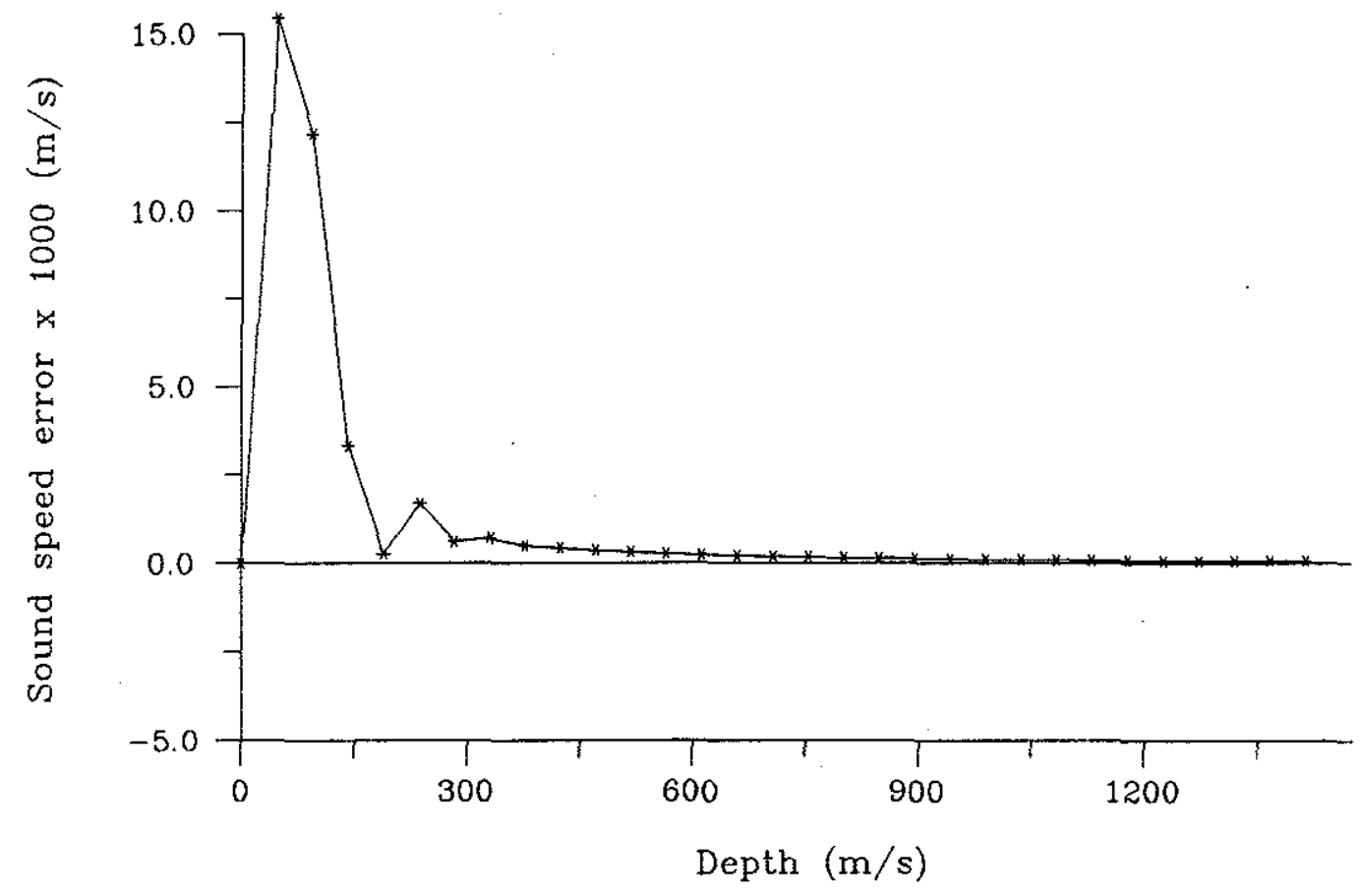

Figure 3-7: Sound speed error versus depth for inversion method 2 applied to the $n^{2}(z)$ linear profile. 


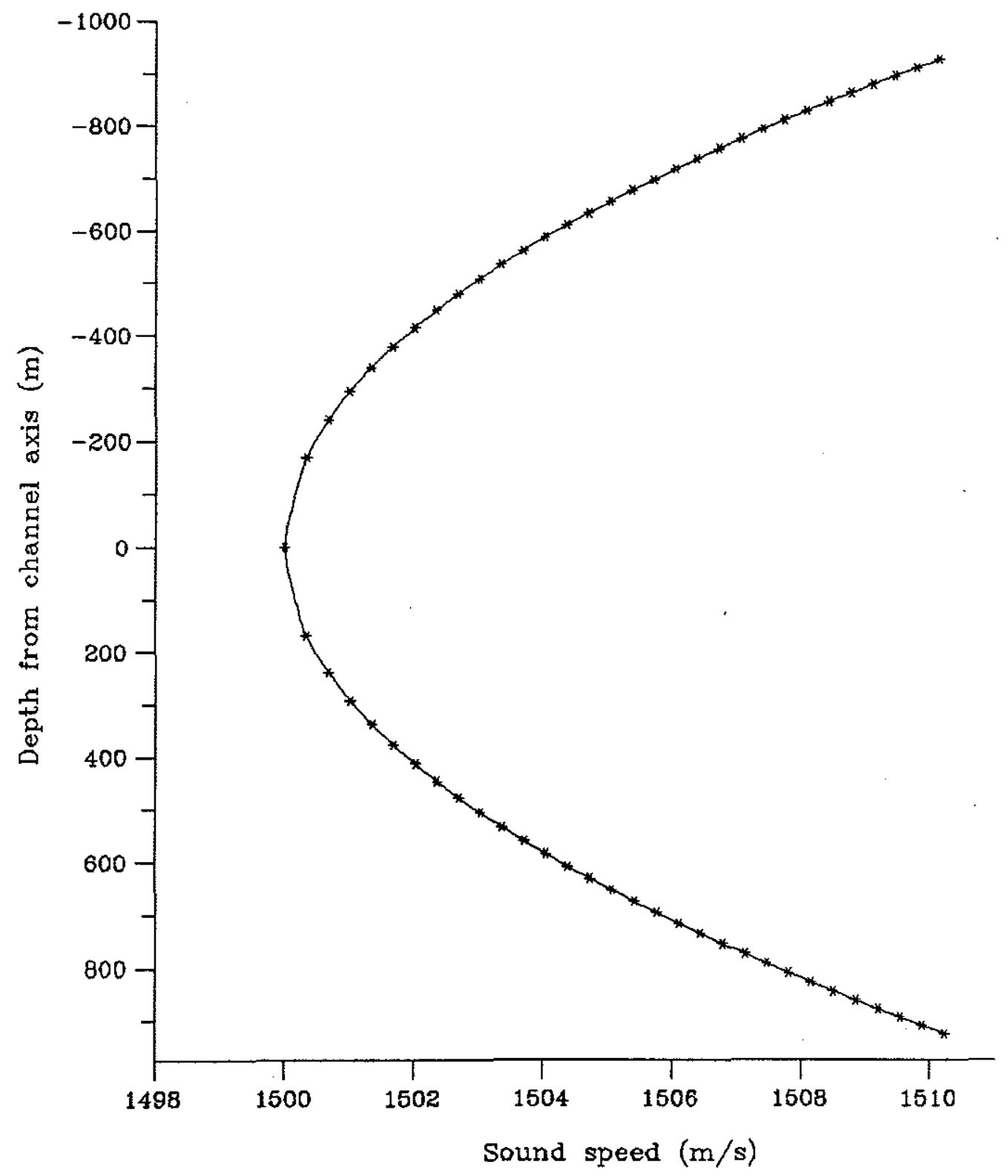

Figure 3-8: Depth versus sound speed for the parabolic profile recovered using inversion method 1. The points below the channel axis are the actual inversion results, and the profile symmetry was used to generate the profile above the axis. 


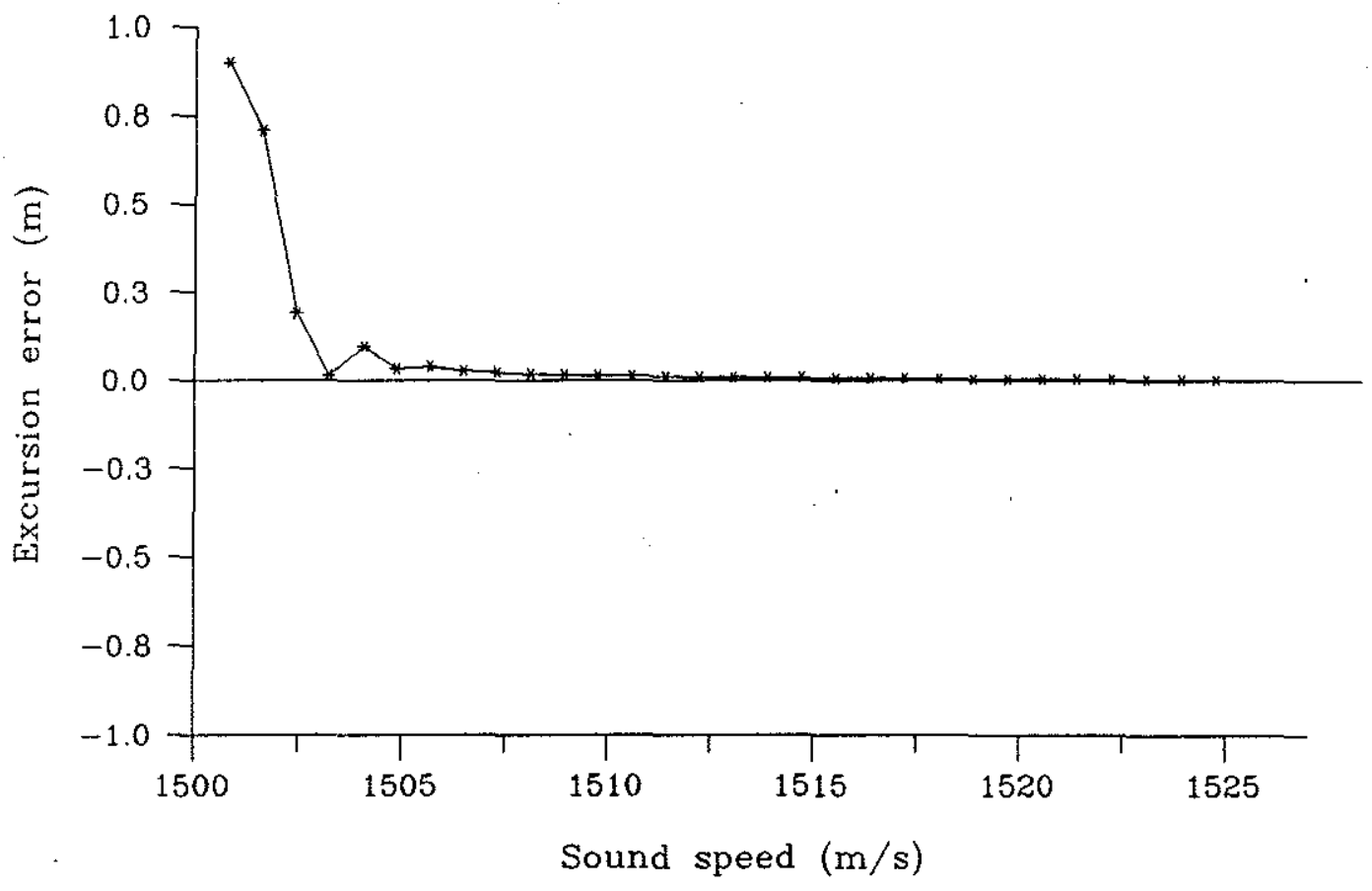

Figure 3-9: Excursion error as a function of sound speed for inversion method 1 applied to the parabolic profile. Excursion error is twice the depth error. 


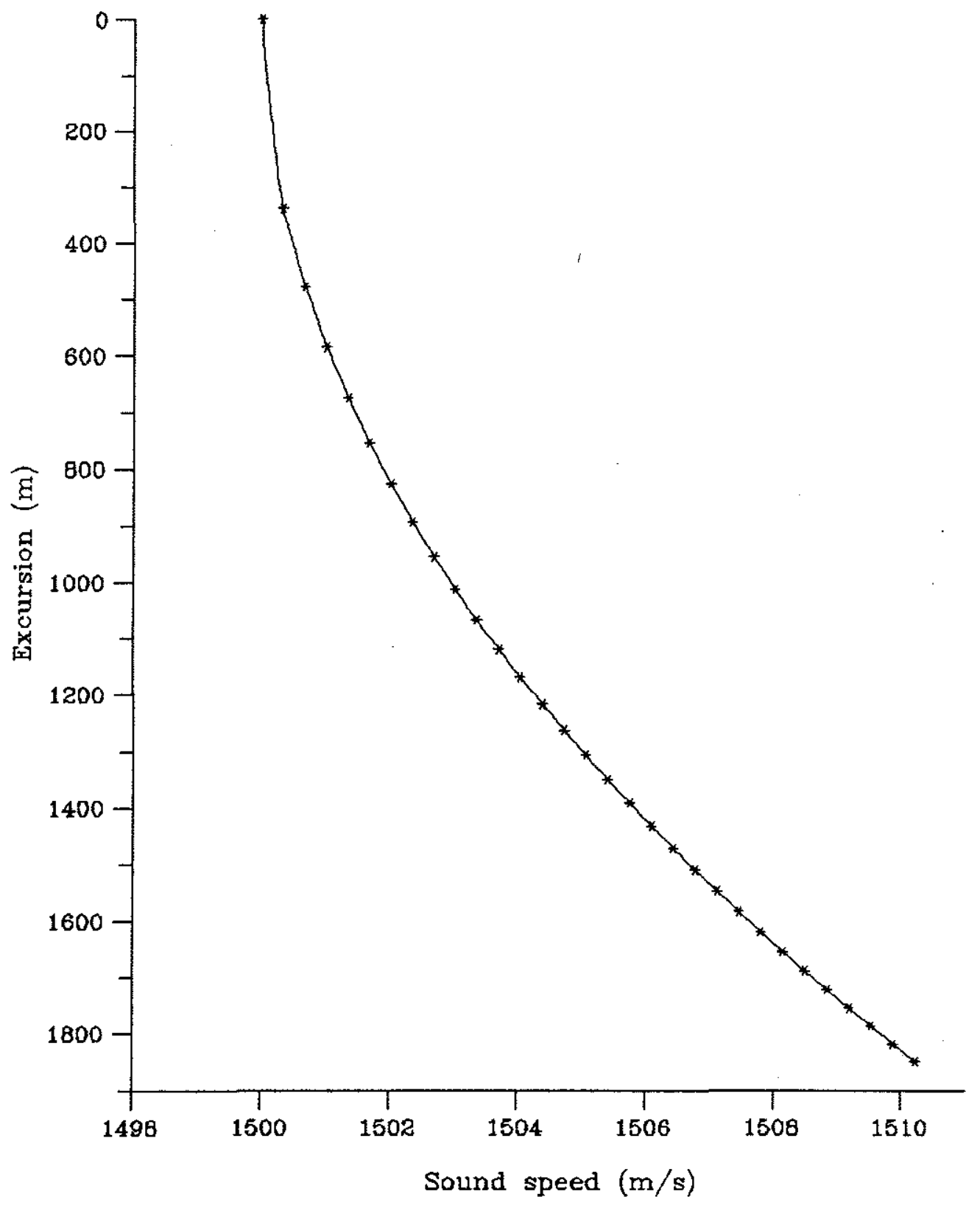

Figure 3-10: The excursion for the parabolic profile computed using inversion method 2. The excursion is the distance between points of equal sound speed on the two branches of the sound speed profile. 


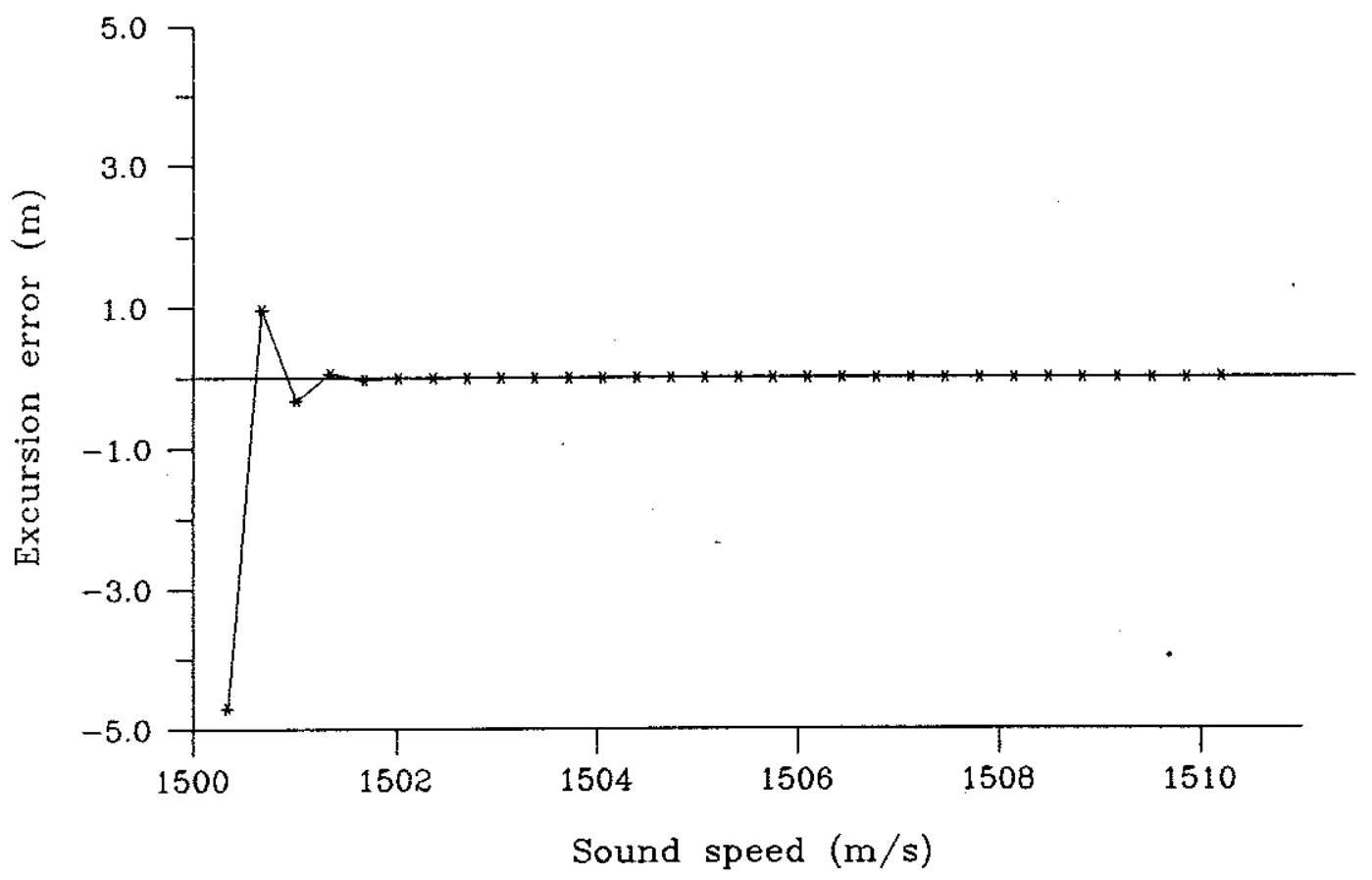

Figure 3-11: Excursion error versus sound speed for inversion method 2 applied to the parabolic profile. 


\subsection{Application of the inversion methods to a bilinear profile}

The sound speed profile in the ocean is determined by the temperature, pressure, and salinity which in general leads to sound speed profiles more complicated than the ideal profiles we have considered. A nominal deep ocean sound speed profile consists of a region near the surface, the thermocline, where the temperature effects dominate and the sound speed decreases from its surface value to a minimum at the axis of the deep sound channel. Below the depth of minimum sound speed, the increase of pressure with depth is the dominant influence, and the sound speed increases with depth. Although the sound speed structure can be quite complicated, we will use a simple bilinear model with a channel axis at 686 meters (figure (3-12) to further illustrate the inversion methods.

The modal eigenvalues for this model were computed for a frequency of $220 \mathrm{~Hz}$ using the SACLANTCEN normal-mode acoustic propagation model program (SNAP)[27]. Initially the data set was truncated before applying inversion method 2 in order to use only modes whose upper turning point is deeper than 100 meters (to avoid effects of interactions with the surface). The resulting excursion as a function of sound speed is shown in figure (3-13), and the excursion error is shown in figure (3-14). The greatest error occurs near the channel axis where the profile smoothness assumptions are not met, and where the extrapolation from the measured data to the assumed point at $\xi=1.0$ is made.

Next we use a data set containing the eigenvalues for normal modes with a lower turning depth shallower than 3200 meters. The initial portion of this data set consists of eigenvalues for modes with two turning points while the eigenvalues at the end of the data set are from modes that interact with the surface and have a lower turning point in the water column. We assume that the value of the sound speed at the surface is available which allows us to determine the value of the horizontal wavenumber separating the two types of modes by setting the WKB phase integral integrand equal to zero so that

$$
\frac{\omega}{c(z)}-k_{r_{n}}=0
$$

For the modes with two turning points $F(\xi)$ is calculated using equation (3.6)

$$
F(\xi)=\pi(n-1 / 2) / k_{0}
$$




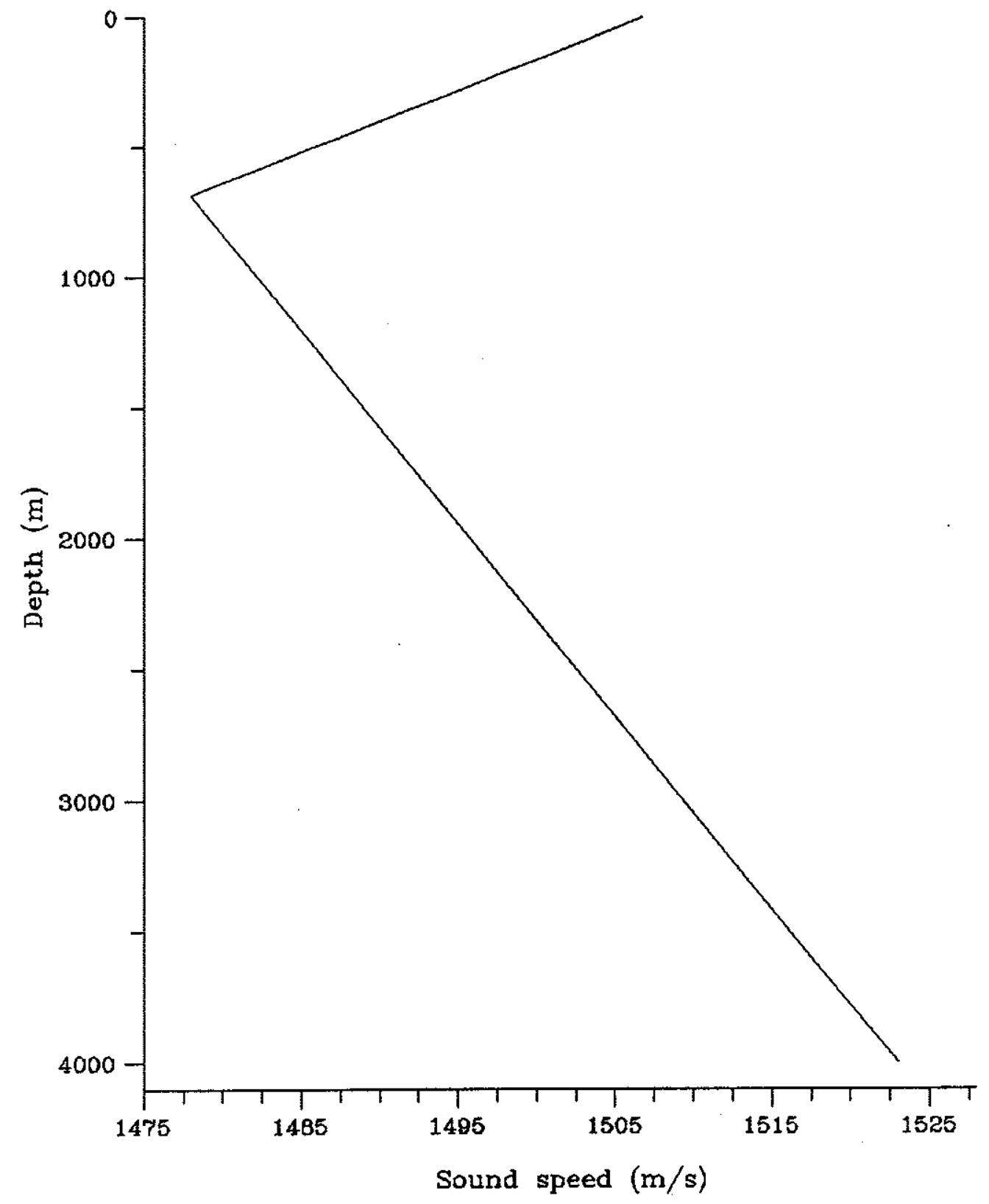

Figure 3-12: The bilinear sound speed profile model. 


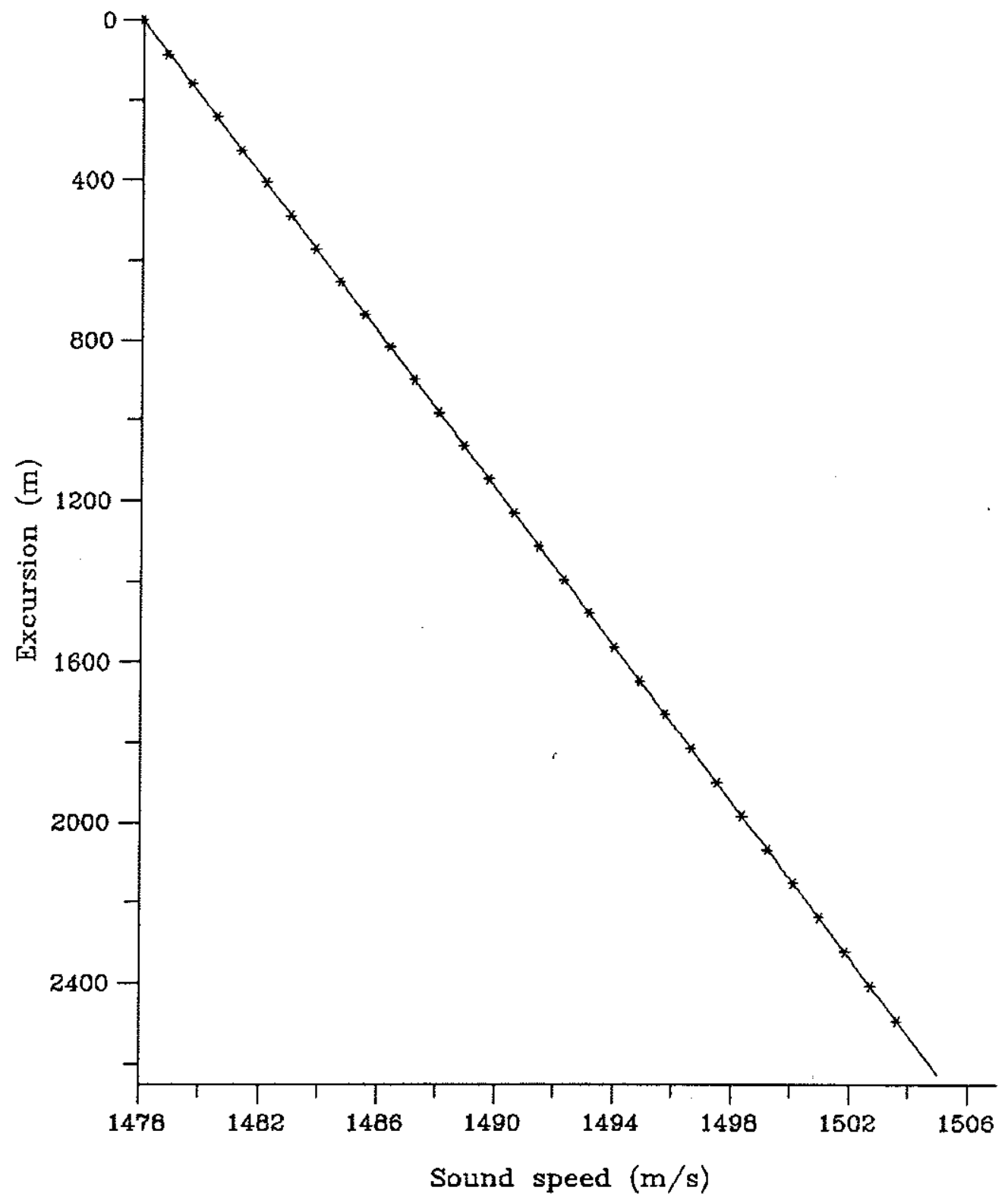

Figure 3-13: The excursion for the bilinear profile using eigenvalues for modes with an upper turning point deeper than 100 meters. 


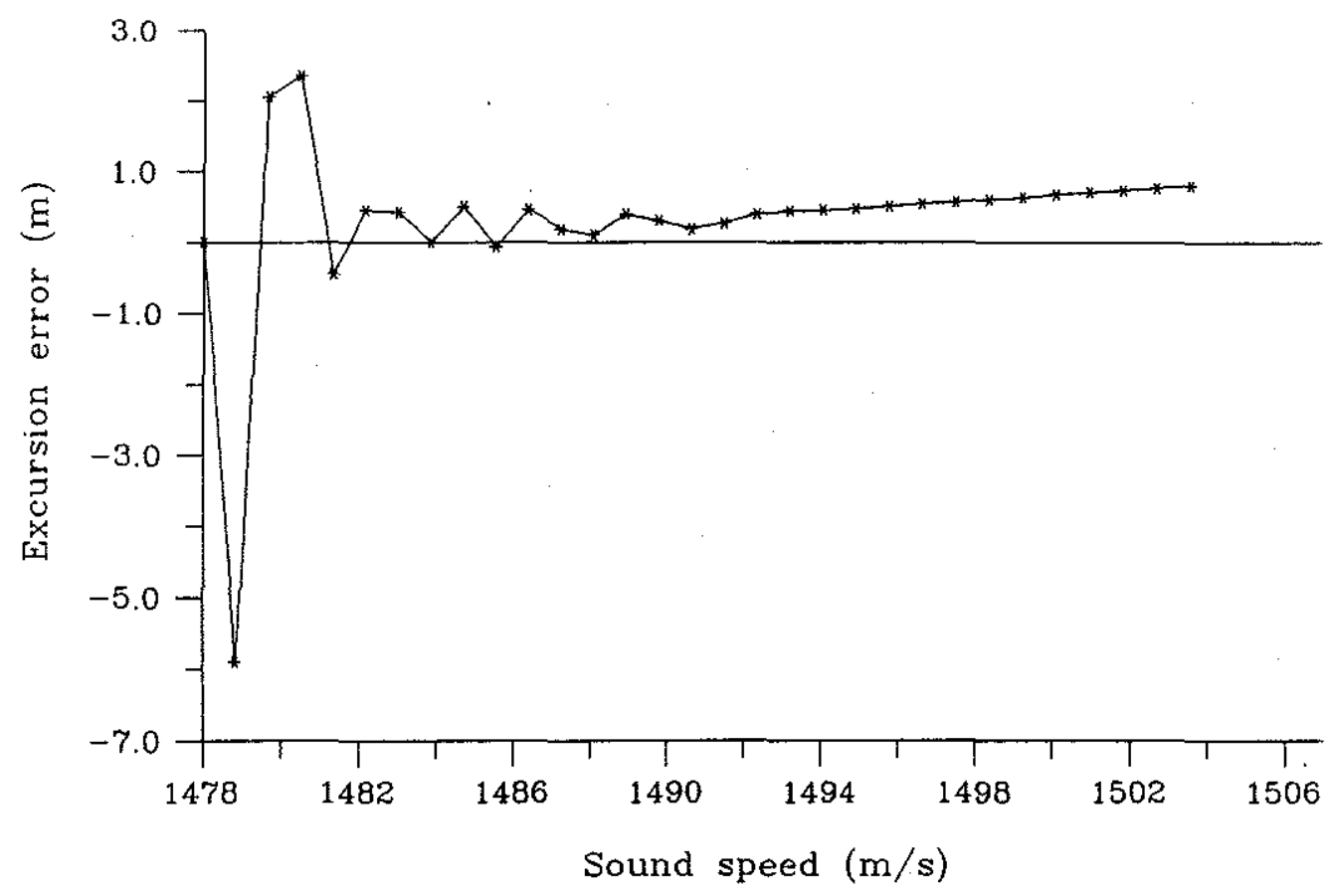

Figure 3-14: Excursion error for the bilinear profile in the region with two turning points.

and for the modes with only one turning point equation (3.4) gives

$$
F(\xi)=\pi(n-1 / 4) / k_{0}
$$

Applying inversion method 2 gives the excursion versus sound speed which in the portion of the profile having two turning points is the difference in depth between points of equal sound speed, and in the portion of the profile with a single turning point is the depth at which the value of sound speed occurs. Figure (3-15) shows the excursion versus sound speed obtained. As before, large errors occur near the channel axis, plus there are large errors at the depth where the transition from two to one turning point is made. This latter error appears to result from errors in the spline fit at the point where the form of $F(\xi)$ changes. If we include a sediment layer with a $1 \mathrm{~m} / \mathrm{s}$ sound speed gradient starting at a depth of 4000 meters, then the inversion using method 2 will give the distance between the two turning points of equal sound speed for sound speeds in the range from $1478 \mathrm{~m} / \mathrm{s}$ to $1507 \mathrm{~m} / \mathrm{s}$ and the actual depth at which the sound speed occurs for sound speeds greater than $1507 \mathrm{~m} / \mathrm{s}$ (figure (3-16)). Consequently we obtain the sediment sound speed profile directly and over 
a portion of the water column we also obtain the sound speed profile directly. Figure (3-17) shows the excursion error over the entire profile. The regions of largest error occur where the form of either the sound speed profile or the function $F(\xi)$ changes. The outputs presented here assume that in addition to the modal eigenvalues, the sound speed at the surface and the minimum sound speed in the profile are available. If the last item is not known, it may be possible to use an iterative procedure to find an approximation to the excursion as a function of sound speed (i.e. find the minimum sound speed) or to separate the portions of the sound speed profiles above and below the channel axis; however, we have not pursued this. The final approach for this profile is to treat it as a symmetric profile by defining an 'equivalent symmetric profile' [35] such that

$$
z_{e s p}= \pm \frac{1}{2}\left(\left|z_{+}\right|+\left|z_{-}\right|\right)
$$

where $z_{+}$and $z_{-}$are the distances above and below the channel axis to points having sound speed $c$. Inversion method 1 is applied to the data for the region with two turning points $(F(\xi)$ must be divided by 2 as in the case of the parabolic profile) to give the portion of the equivalent symmetric profile below the channel axis, and symmetry is used to generate the portion above the axis. Figure (3-18) illustrates the results of using this method which, in a sense, is averaging the vertical phase contributions of the two portions of the sound speed profile. 


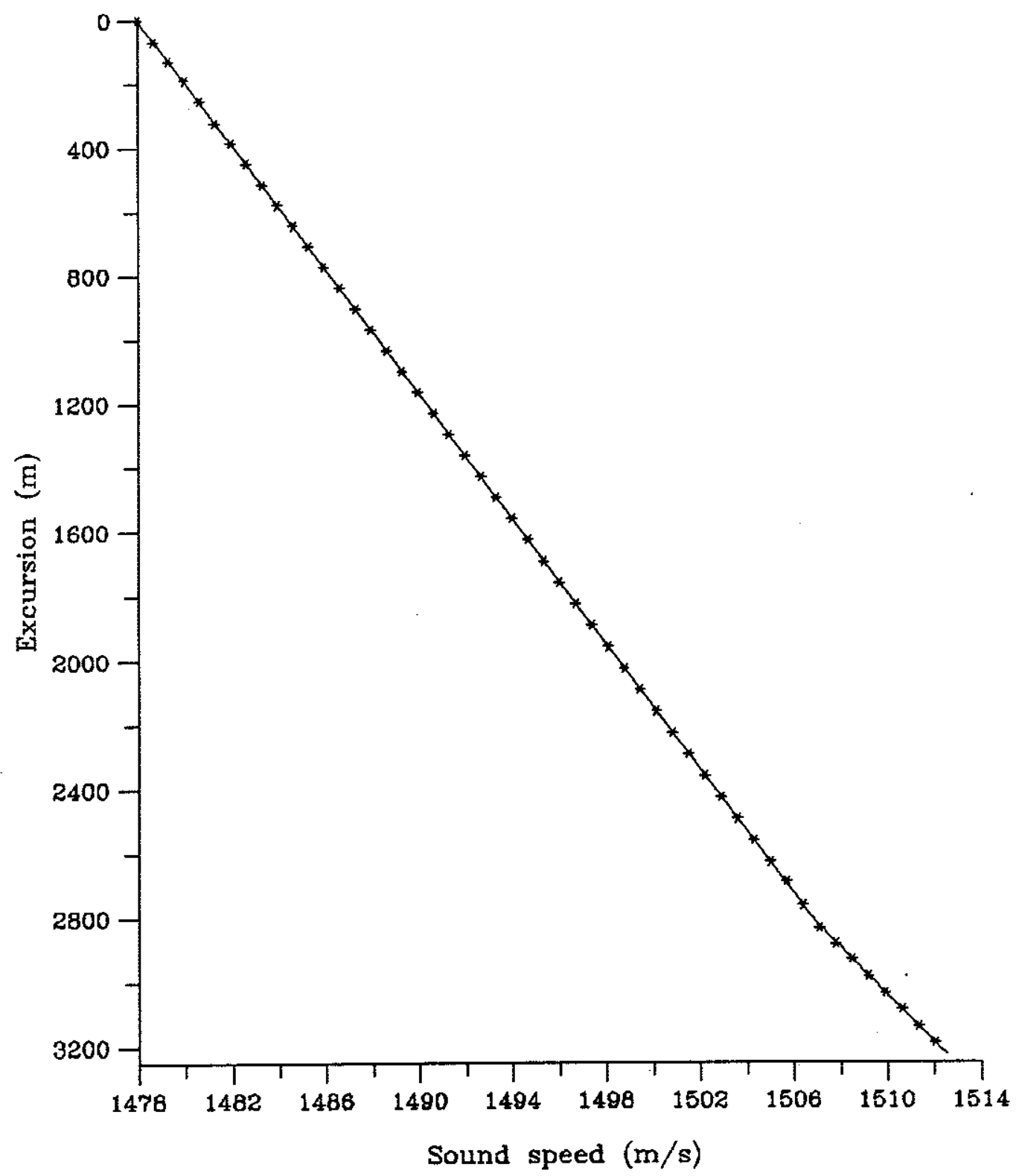

Figure 3-15: Excursion versus depth for the bilinear profile using eigenvalues for modes turning above 3200 meters. Below 2813 meters this curve is the sound speed profile. 


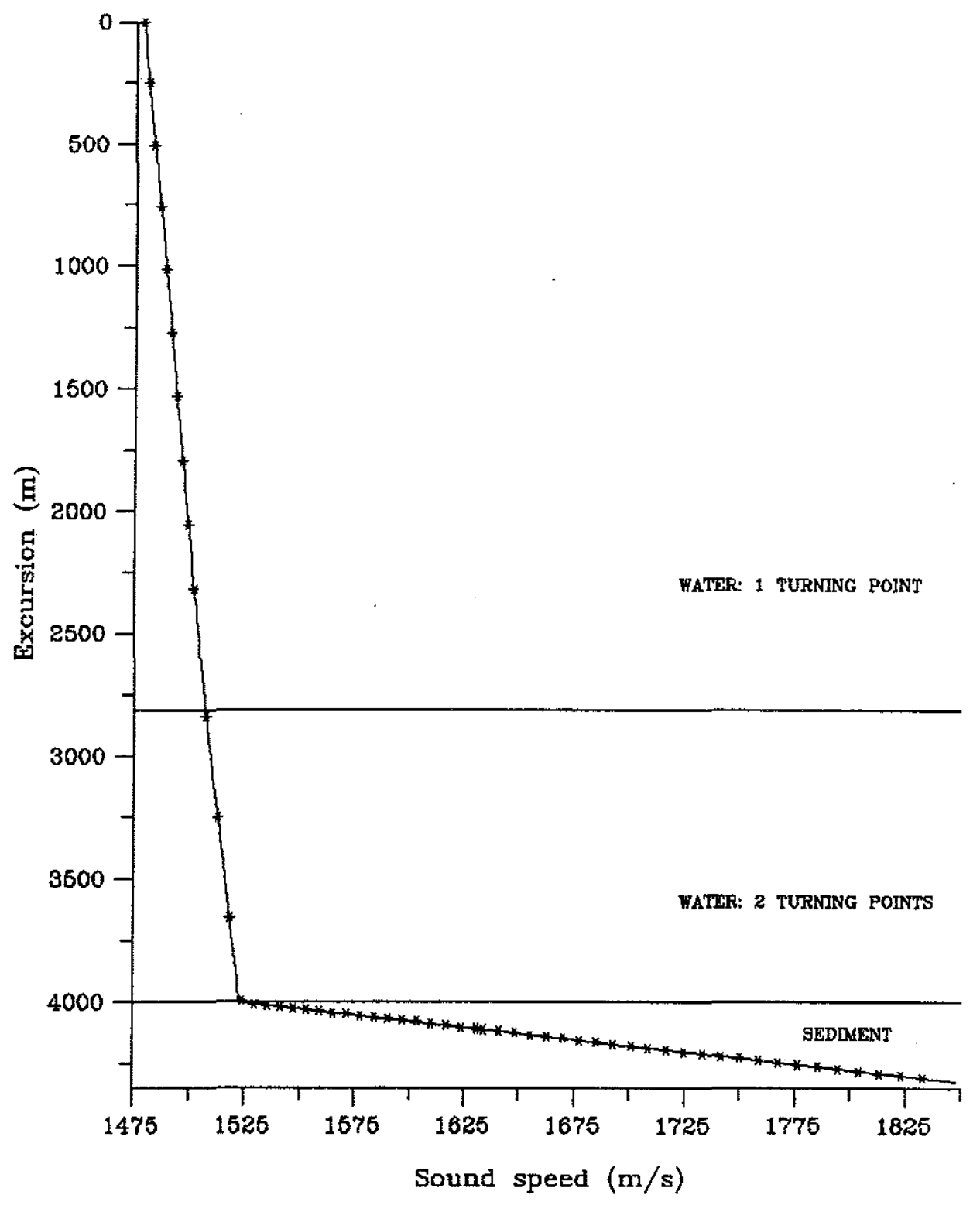

Figure 3-16: Excursion versus depth for the bilinear profile. Below 2813 meters this curve is the sound speed profile. A frequency of $140 \mathrm{~Hz}$ was used for this inversion to provide bottom interacting modes. 


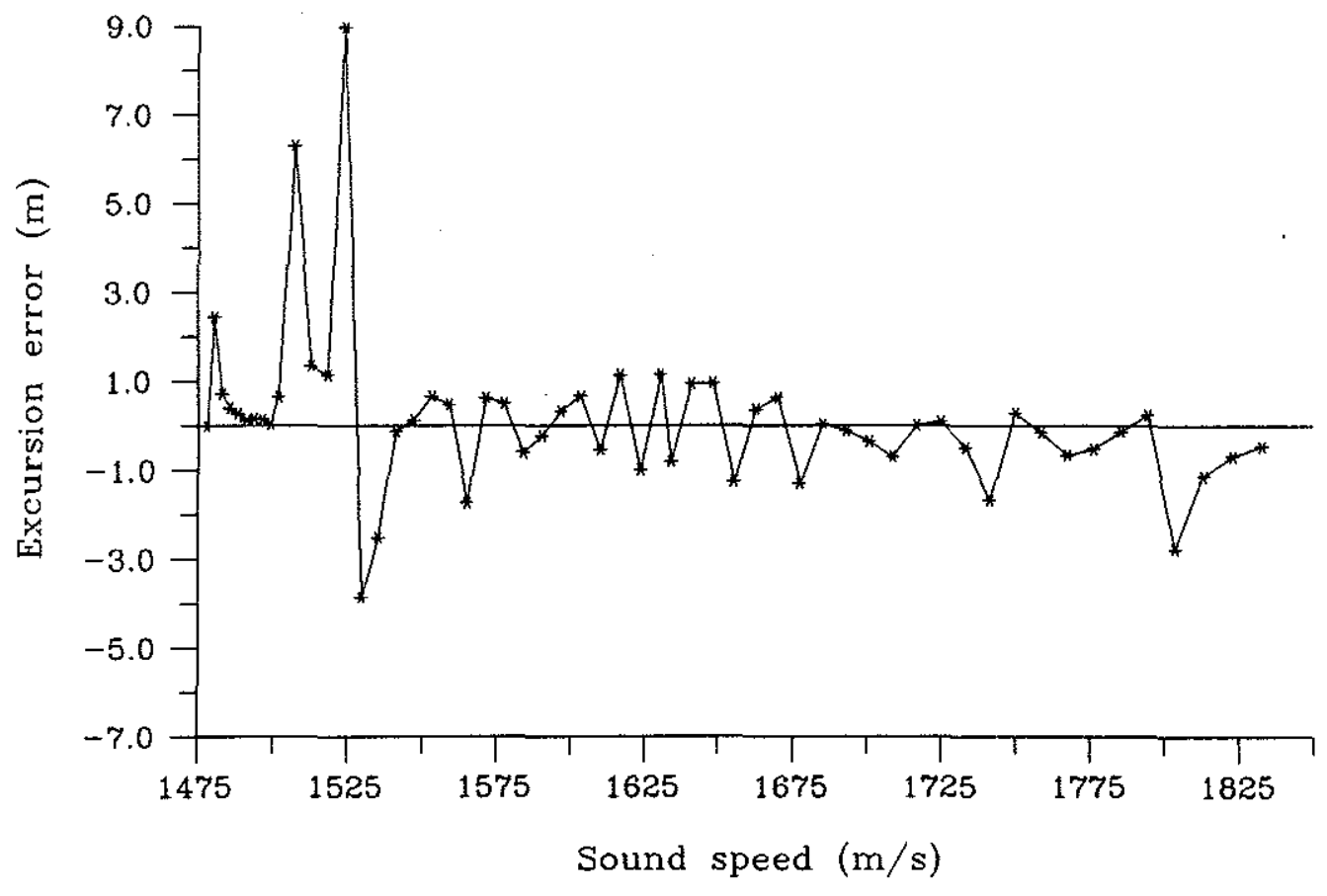

Figure 3-17: Excursion error as a function of sound speed for the bilinear profile. For sound speeds greater than $1507 \mathrm{~m} / \mathrm{s}$ this is depth error. 


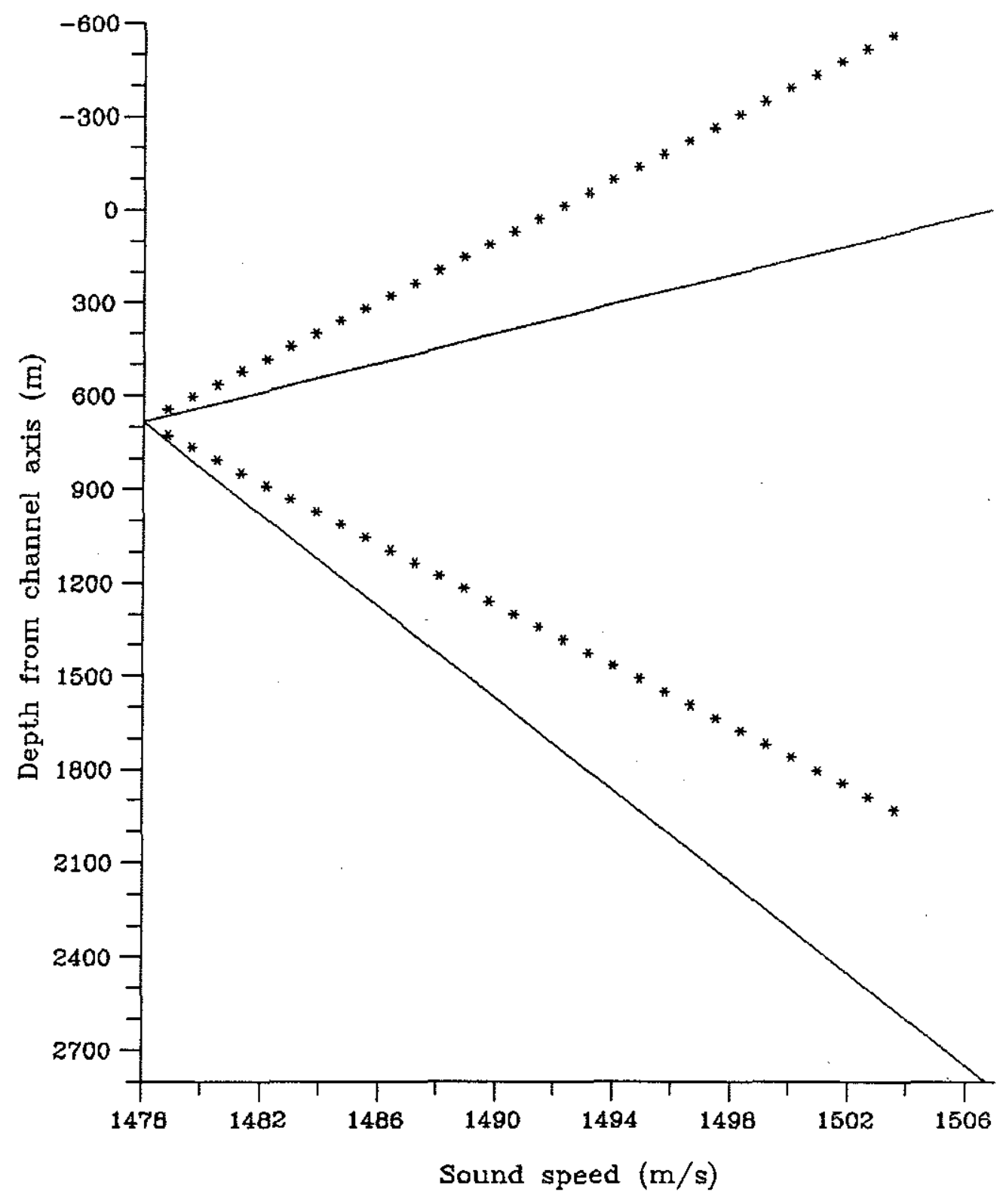

Figure 3-18: Sound speed versus depth for the symmetric profile equivalent to the bilinear profile. 


\subsection{Performance of the inversion methods}

For any inverse method, questions of practical interest concerning the solution are:

- Is the solution unique?

- What is the resolution of the inversion?

- How well does the inversion method perform with imperfect or noisy data?

\subsubsection{Uniqueness}

The question of uniqueness for Sturm-Liouville problems has been extensively studied ( see for example [29], [32]), and the requirements for unique recovery of the coefficients are well known. If we model the problem as a horizontally stratified medium consisting of the water column, fluid sediment layers, and a semi-infinite halfspace which can be considered as providing an impedance boundary condition, then the problem can be written on a finite interval $[0,1]$ as

$$
\begin{gathered}
\frac{d^{2} v}{d s^{2}}+(\lambda-q(s)) v=0 \\
\sin \alpha v(0)+\cos \alpha v^{\prime}(0)=0 \\
\sin \beta v(1)+\cos \beta v^{\prime}(1)=0
\end{gathered}
$$

The general problem is to find $\alpha, \beta$, and $q$ from spectral data. The basic data set is the infinite set of eigenvalues $\lambda_{1}<\lambda_{2}<\ldots$ for the given Sturm-Liouville problem. If the potential is symmetric about $s=1 / 2$ and $\alpha=\pi-\beta$ this is sufficient to provide for unique recovery of $\alpha$ and $q$; however, for a general profile, an additional infinite sequence of data will be required. Three different possibilities for this second set of data include [32]

1. a second set of eigenvalues $\hat{\lambda_{1}}<\hat{\lambda_{2}}<\ldots$ where a different boundary condition is applied in place of equation (3.47), i.e. $\beta$ would be replaced by $\hat{\beta}$;

2. the set of normalization constants $\delta_{n}=\left\|v_{n}\right\|^{2} /\left[v_{n}(0)\right]^{2}, n=1,2, \ldots$, when $0<\alpha<\pi$ or $\delta_{n}=\left\|v_{n}\right\|^{2} /\left[v_{n}^{\prime}(0)\right]^{2}$ when $\alpha=0 . v_{n}$ is the eigenfunction for the eigenvalue $\lambda_{n}$, and $\left\|v_{n}\right\|^{2}=\int_{0}^{1}\left[v_{n}(s)\right]^{2} d s$ 
3. the set of ratios $v_{n}(1) / v_{n}(0)$ when $0<\alpha, \beta<\pi$ or $v_{n}^{\prime}(1) / v_{n}^{\prime}(0)$ when $\alpha=\beta=0$, or similar ratios for $\alpha=0,0<\beta<\pi$ or $\beta=0,0<\alpha<\pi$.

In addition, Hochstadt and Lieberman [26] show that uniqueness is possible given the set of eigenvalues $\lambda_{1}<\lambda_{2}<\ldots$ and knowledge of $q(s)$ on the interval $[1 / 2,1]$.

For the problem considered here, we have one partial set of eigenvalues and knowledge of one boundary condition (the pressure release surface). Conseqently, we cannot expect that the answer provided by the inverse methods will be unique. From a practical standpoint this means that there may be additional information on a finer verticle scale than the frequency of our experiment could measure, but which could be obtained at a different frequency; or there may be a variety of models possible for the region below the depth of deepest penetration of the rays equivalent to the modes used for the inversion which would give the same finite set of eigenvalues as we have measured. It should be noted that, if horizontal wavenumbers for two (or more) frequencies are used, the transformation to the variable $\xi$ will map all the eigenvalue data to the inerval $[0,1]$, and all the data will fit on the same curve $F(\xi)$ since $F(\xi)$ is normalized. This allows us to combine data obtained using two frequencies.

\subsubsection{Resolution}

One of the advantages of perturbative inversion methods is that the linear inverse theory used allows a quantitative estimate of the resolution provided by the data (for a discussion of linear inverse theory see [33]). With these nonlinear inversion methods we can only provide a qualitative description of the depth resolution. Inversion method 1 uses the function $H(\xi)$ obtained by interpolating and differentiating the input data to find the depth at which a particular value of the index of refraction will cause a ray with angle $\sin ^{-1} \sqrt{\xi}$ to turn. We can expect that the resolution will be related to the depth difference between turning depths for successive modes. Better resolution should be obtained if the sound speed profile and the frequency are such that the turning depths of adjacent modes are closely spaced. The turning depths are found using the condition

$$
\frac{\omega}{c(z)}=k_{r_{n}},
$$

and high frequencies or large sound speed gradients give more closely spaced turning depths. In integrating over $\kappa$ to obtain the depth for a given index of refraction, we divided the range 
of $\kappa$ uniformly, and the output values from the inversion will be closer together in portions of the sound speed profile with the highest gradients. This can be seen in figure (3-16).

Inversion method 2 approaches the problem in a similar manner finding the difference in depth between points on the profile having the same sound speed, and similar arguments concerning the spacing of the mode turning points pertain to the depth resolution of this method.

\subsubsection{Performance of the inversion methods in the presence of noise}

Because of the nonlinearity of the problem, it is difficult to obtain an analytic expression giving an indication of how the inversions will perform when the input data is not exact. For inversion method 1, we obtained (equation (3.18)) the Abel integral equation

$$
\frac{d F(\xi)}{d \xi}=H(\xi)=-\frac{1}{2} \int_{0}^{z_{t}} \frac{d \dot{ }}{[\kappa(z)-\xi]^{1 / 2}}
$$

and a similar Abel equation was obtained in method 2 where, rather than inverting the equation, we differentiated with respect to $\xi$ to obtain the desired result, equation (3.29) (the excursion). We have considered that the values of $F(\xi)$ are exact, and, while some noise will be introduced in differentiation due to the inexactness of the values $\xi$ associated with $F(\xi)$, the noise enters primarily in the kernel of the integral equation. Thus we will approach the question of noise effects by numerically testing the inversions using the $n^{2}(z)$ linear profile as a test case.

In carrying out an experiment to measure the eigenvalues in the ocean waveguide, the environment and the instruments will introduce noise into the data, the process of computing the eigenvalues will have some inherent resolution limit imposed by array size and spatial sampling factors, and the computation process will introduce numeric inaccuracies such as round-off error. Rather than attempt to quantify all these factors which will vary from experiment to experiment, we will assume that we have data with random errors of known maximum value. This noise will be modelled as an additive roundoff error with a uniform probability distribution from $-5.0 \times 10^{-n}$ to $5.0 \times 10^{-n}$ where $n$ is the largest decimal position in the eigenvalue affected by the noise. The two inversion methods were run with varying error magnitudes using both the least squares basis spline routines and the cubic smoothing spline routines relying on cross validation to choose the smoothing parameter. Figures (3-19) 


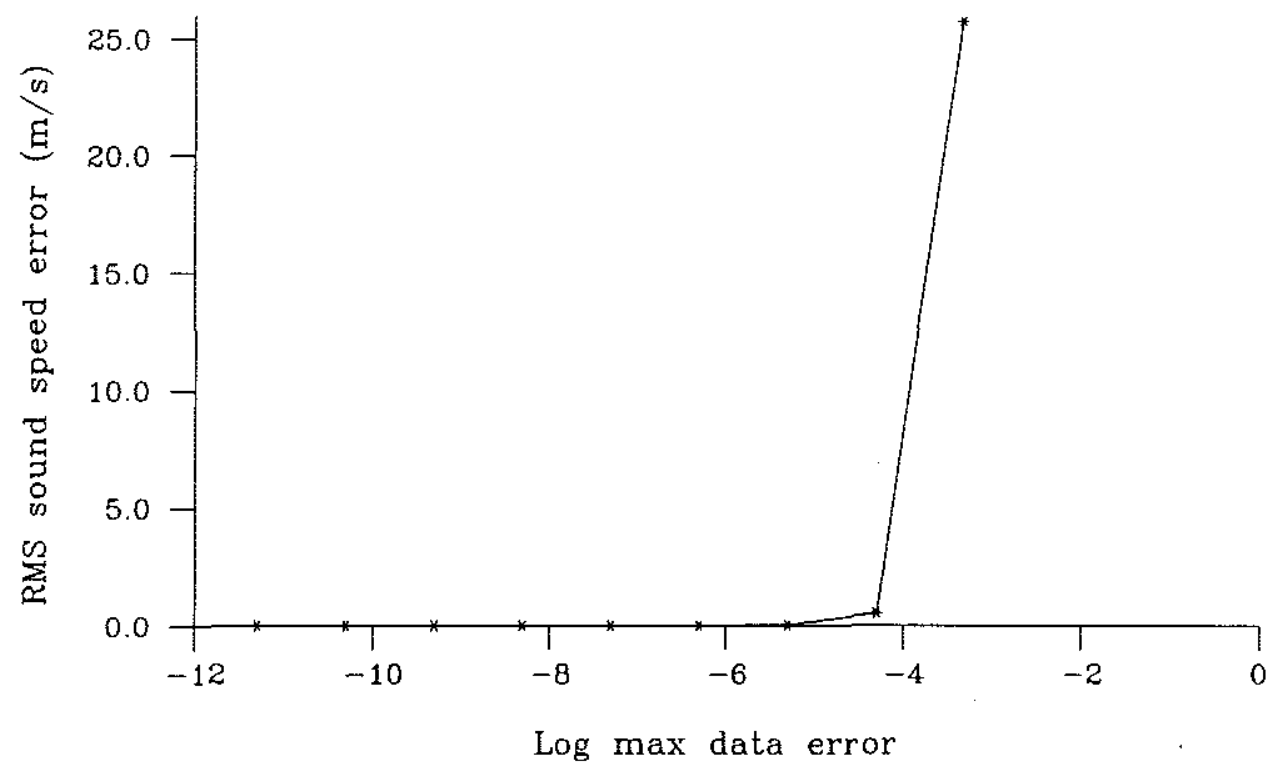

Figure 3-19: RMS error for inversion method 1 using least squares basis splines with noisy data.

through figure (3-26) plot the root mean square error and the absolute value of the maximum inversion errror as functions of the logarithm of maximum introduced error. The results show that the smoothing spline can handle a greater magnitude of noise, and that both inversion methods can handle reasonable amounts of noise in the data. If a mode is missed, i.e. the mode spacing and noise are such that one mode is missed and all subsequent modes are incorrectly numbered by one, then the errors in $F(\xi)$ will produce errors in the output. Figure (3-27) illustrates the error produced when the eigenvalue for mode 25 of the $n^{2}(z)$ linear profile is deleted, and the remaining 24 eigenvalues misnumbered. The sudden jump in $F(\xi)$ leads to a sharp jump in the error of the output at the depth corresponding to the missing mode, and then the error decreases tending to a constant value. 


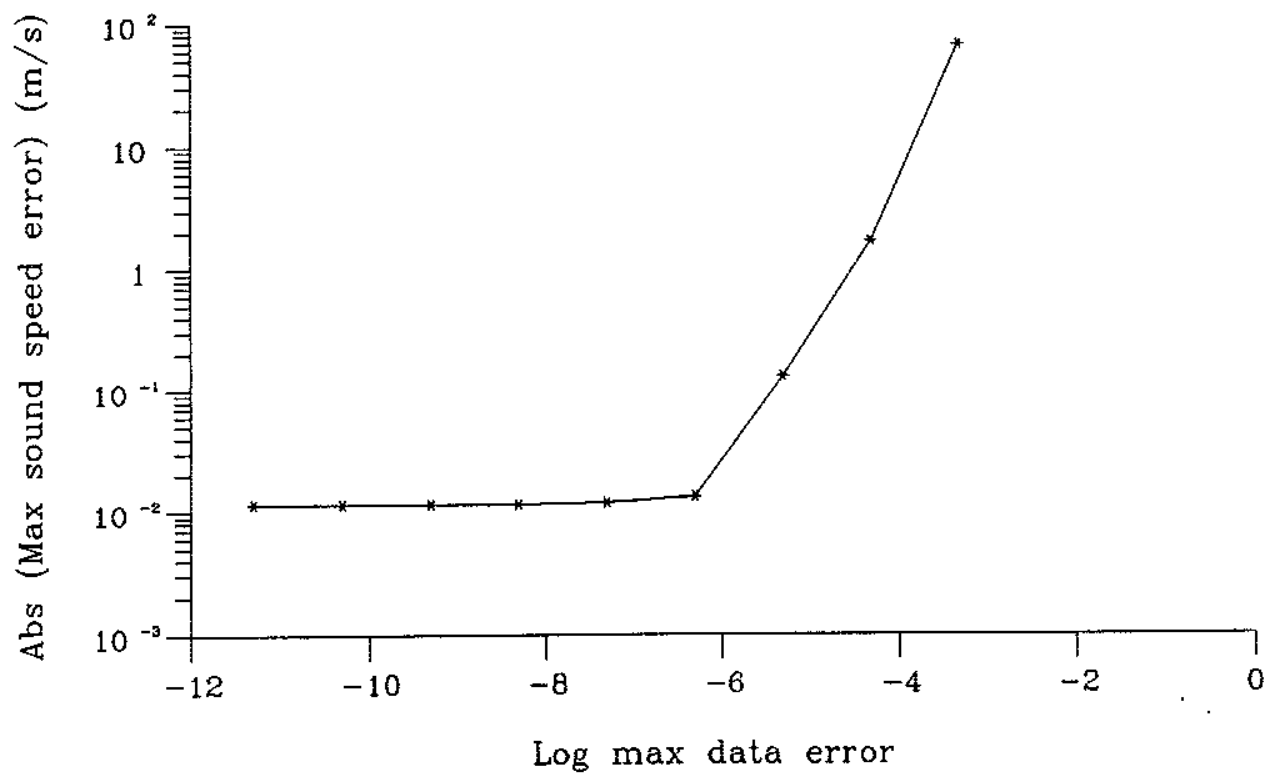

Figure 3-20: Absolute value of the maximum error for inversion method 1 using least squares basis splines with noisy data.

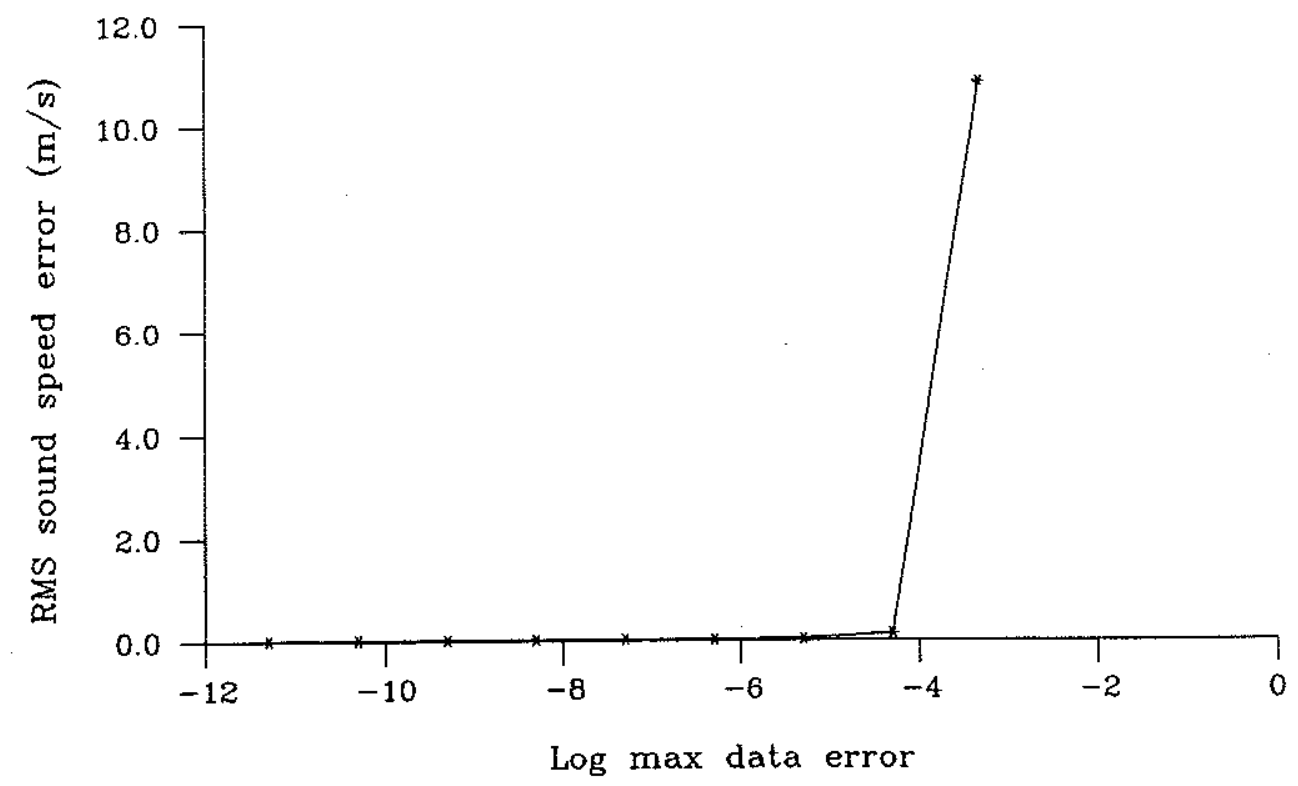

Figure 3-21: RMS error for inversion method 2 using least squares basis splines with noisy data. 


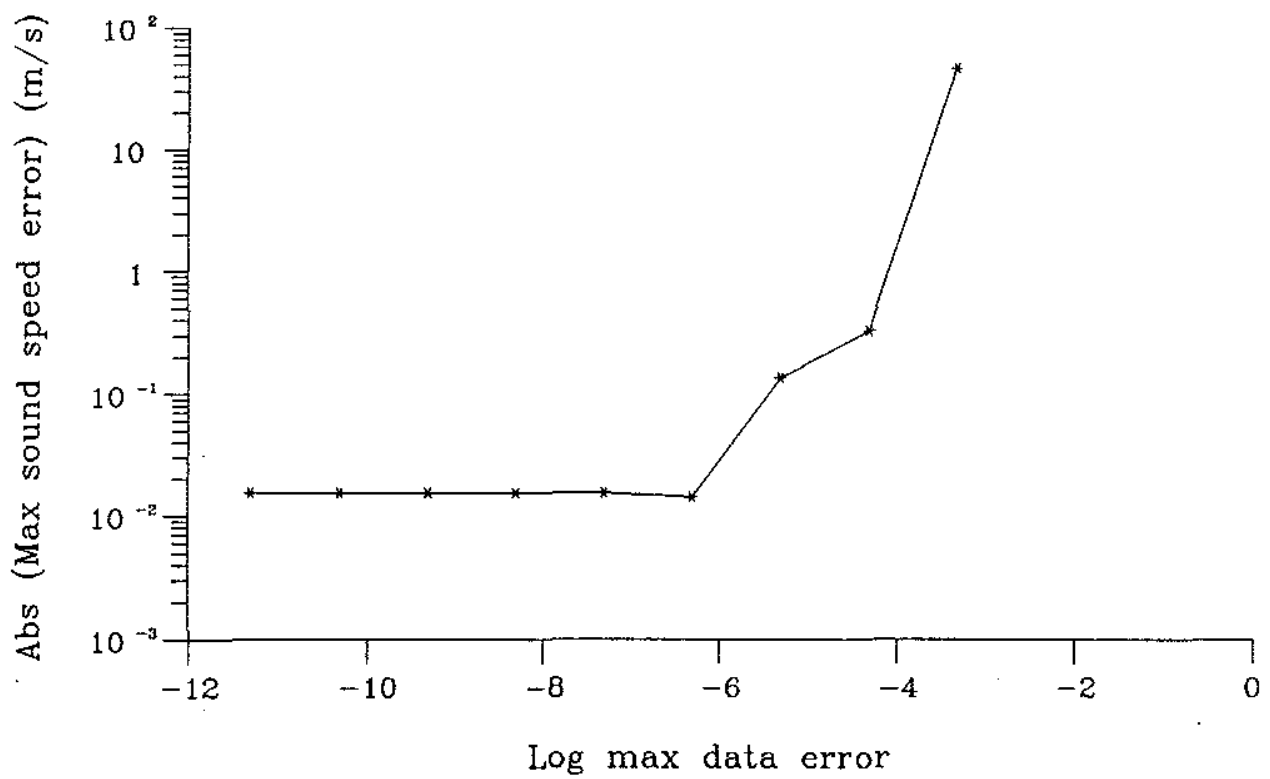

Figure 3-22: Absolute value of the maximum error for inversion method 2 using least squares basis splines with noisy data.

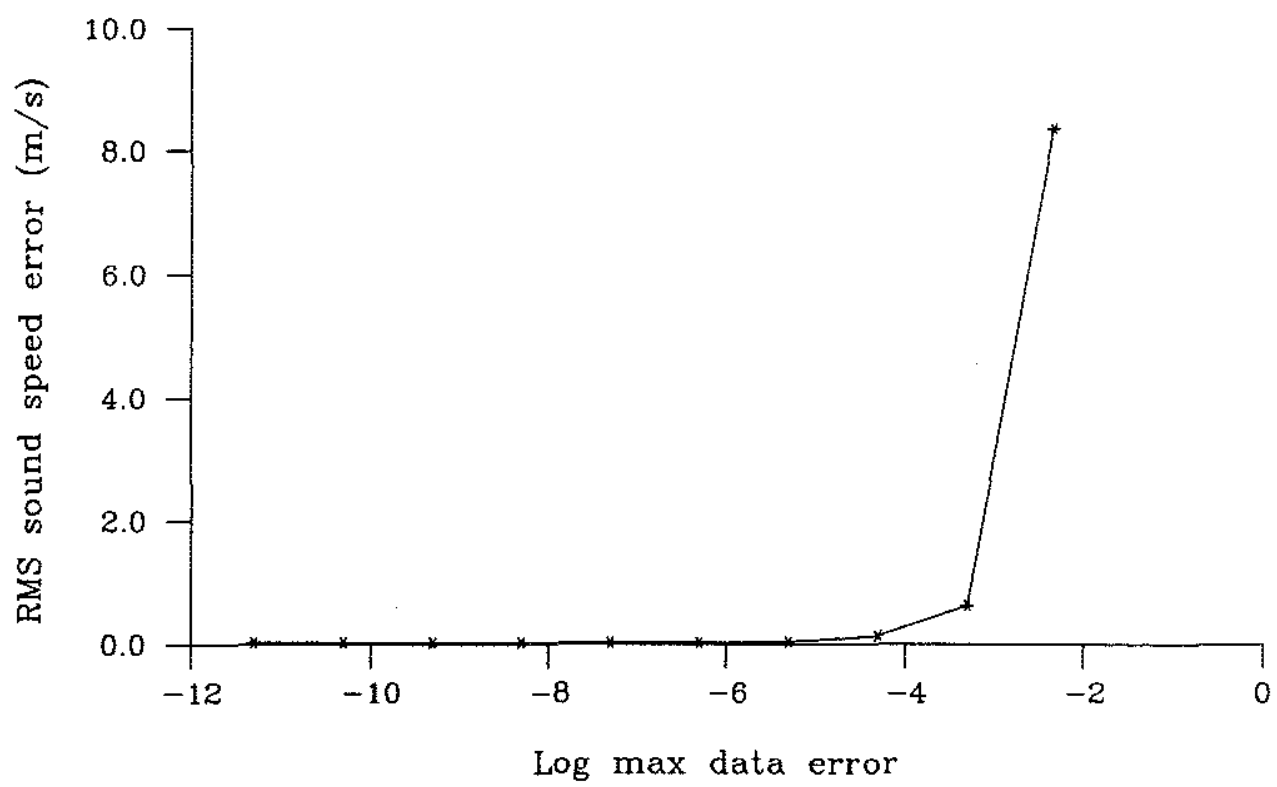

Figure 3-23: RMS error for inversion method 1 using smoothing splines with noisy data. 


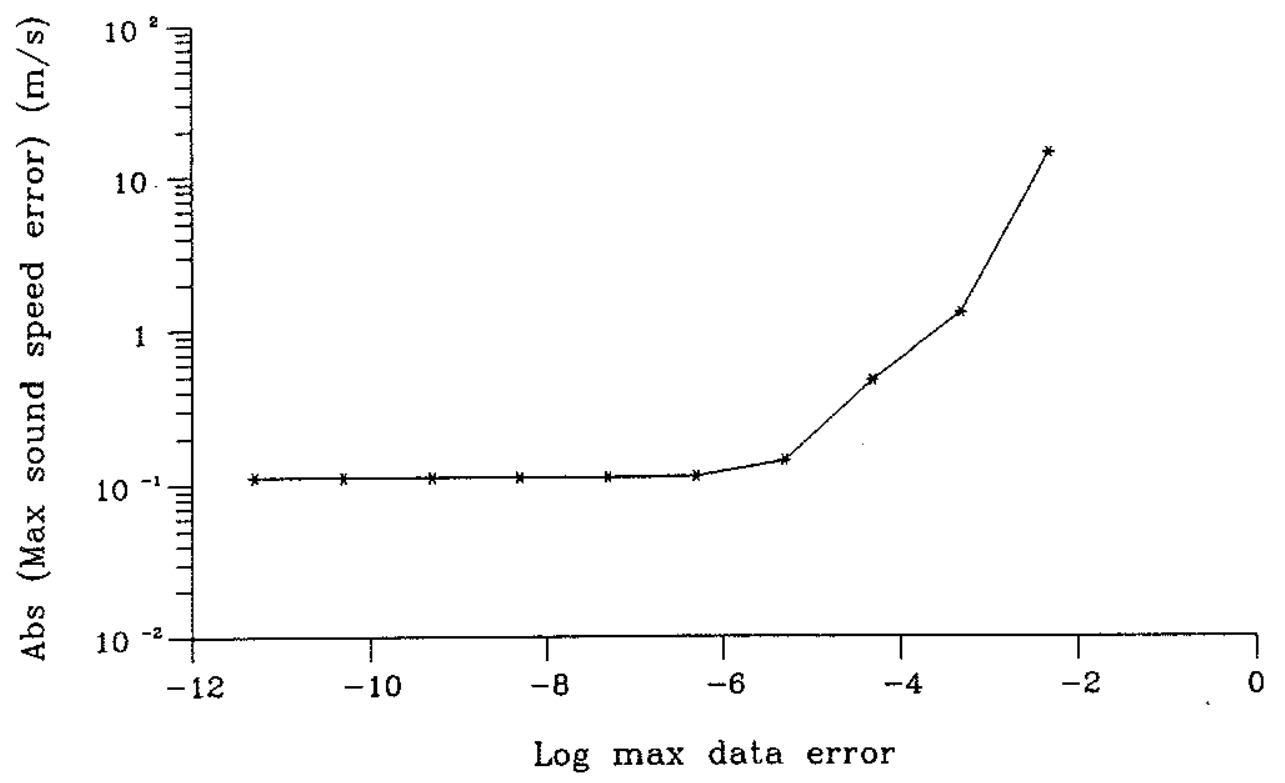

Figure 3-24: Absolute value of the maximum error for inversion method 1 using smoothing splines with noisy data.

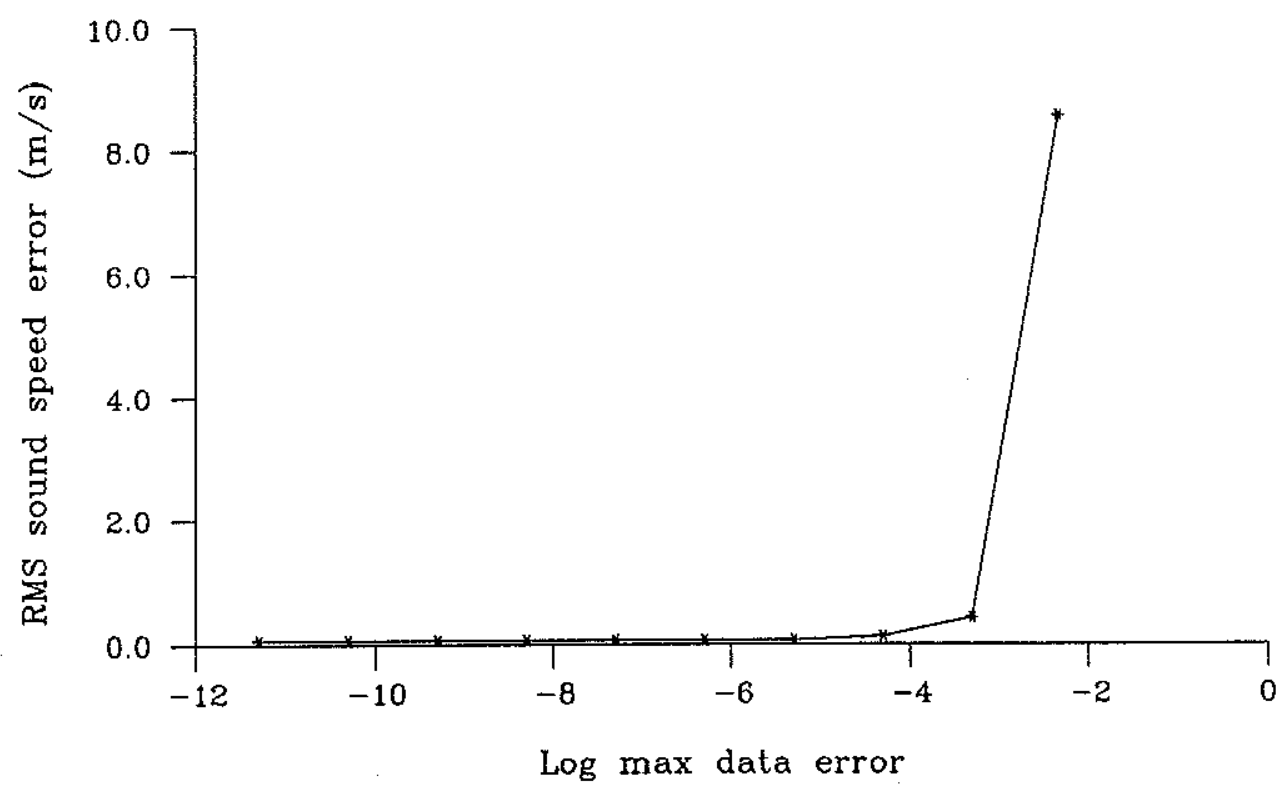

Figure 3-25: RMS error for inversion method 2 using smoothing splines with noisy data. 


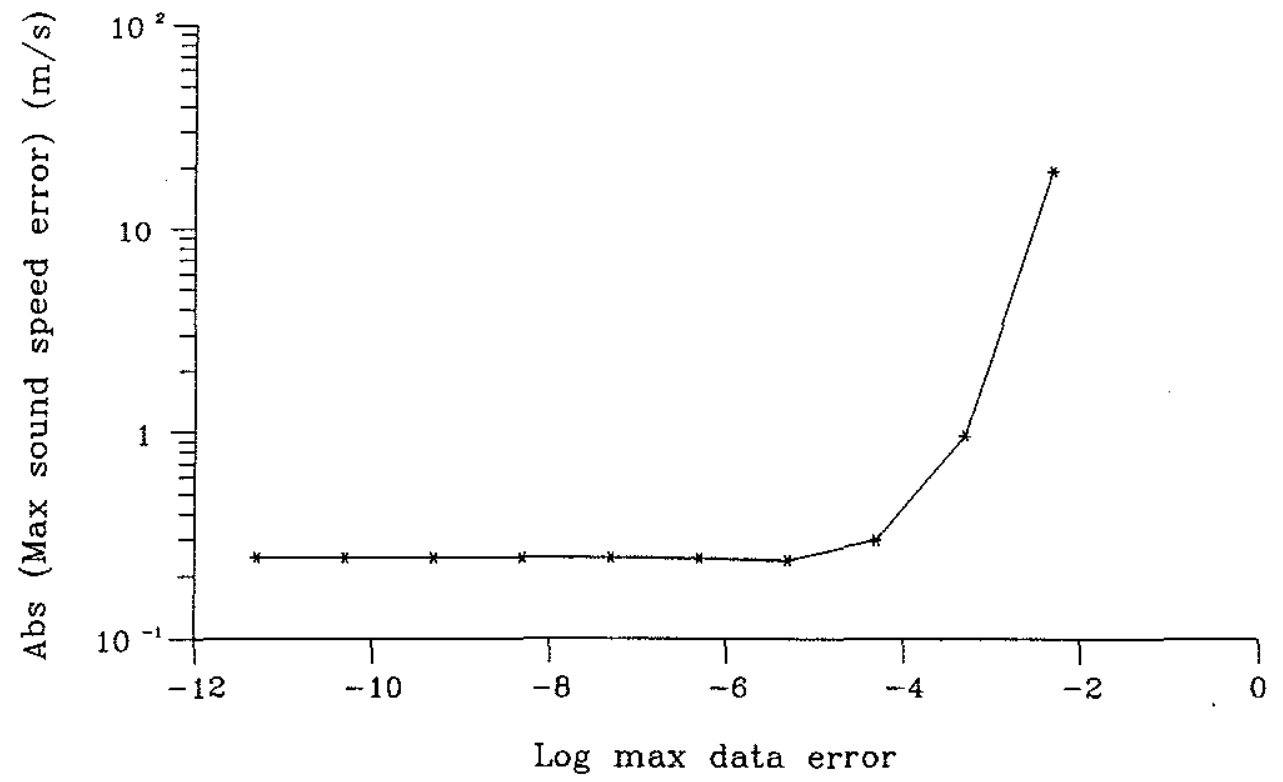

Figure 3-26: Absolute value of the maximum error for inversion method 2 using smoothing splines with noisy data.

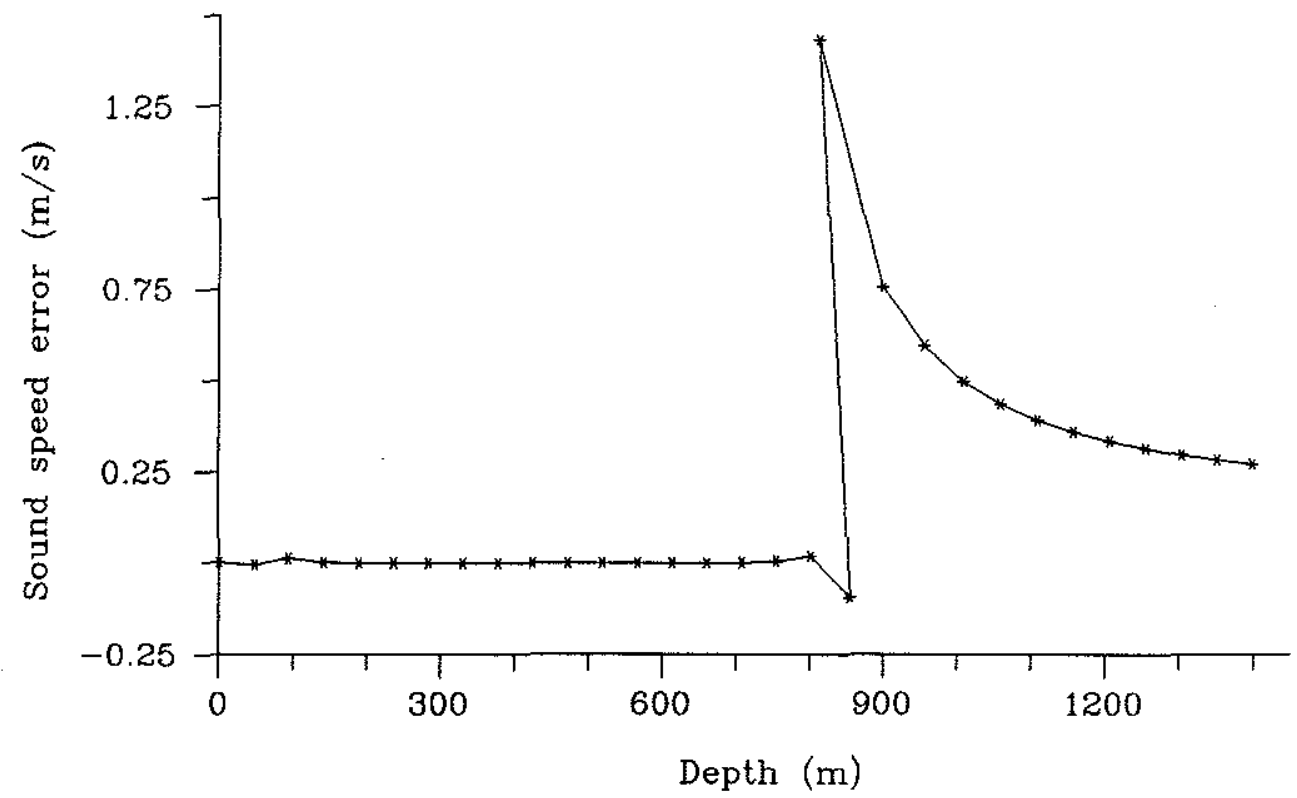

Figure 3-27: Sound speed error for the $n^{2}(z)$ linear profile with mode 25 eigenvalue removed, and higher modes incorrectly identified. 


\section{Chapter 4}

\section{Application to a Shallow Water Waveguide}

In order to illustrate the application of this inversion technique to a specific problem in underwater acoustics, we will consider the problem of determining the acoustic properties of the sediments in a shallow water waveguide. The objective is to provide a portion of the geoacoustic model for the waveguide. A complete geoacoustic model [25] includes information on the geology and topography of the bottom, density profiles, shear wave properties, and compressional wave properties. This inverse method is capable of providing estimates for compressional wave speed and density profiles, although we can only illustrate application to finding compressional wave speed profiles.

The data for the inversion is obtained from an experiment [20], [30] in which a continuous wave source at a fixed depth is towed away from a pair of moored receivers which record sound field pressure (amplitude and phase) as a function of range (see figure 4.1). In actually carrying out the experiment, one source operating at two different frequencies is used which, in principle, allows determination of the density as well as sound speed. The pressure field is sampled at least every half wavelength in range over an aperture of several kilometers, and estimates of the horizontal wavenumbers for the propagating sound field are obtained from the pressure field measurements either by using Prony's method [15] which models the field as a sum of complex exponentials, or by numerically Hankel transforming the measurements to obtain the depth dependent Green's function. In previous work, the bottom properties have been determined using the data from this type of experiment [21] by assuming a geoacoustic model, computing the theoretical Green's function for the model, and calculating the mean 


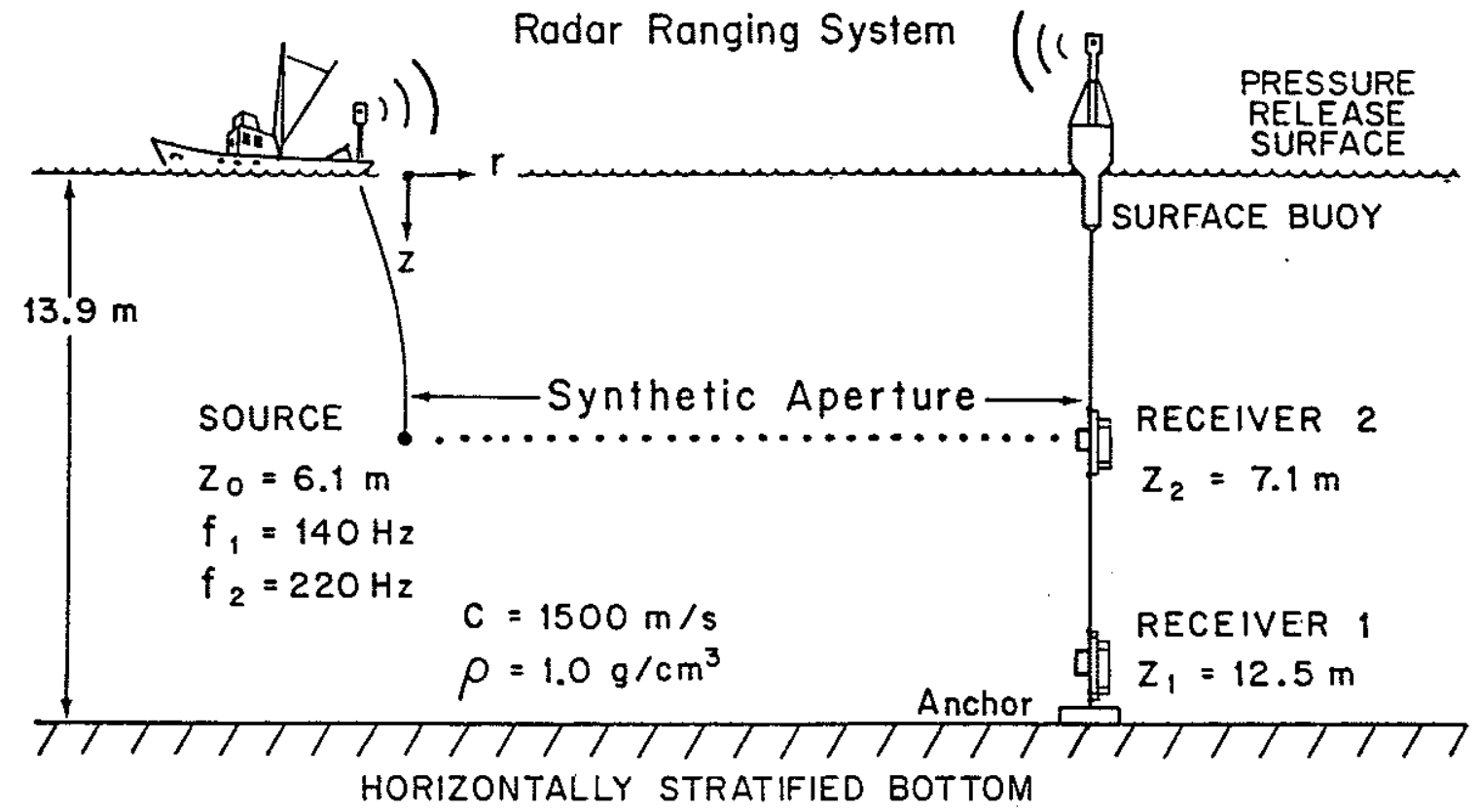

Figure 4-1: Experimental configuration for measuring the eigenvalues in a shallow water waveguide. The parameters shown are for an experiment in Nantucket Sound, Massachusetts [21]

square difference between the two Green's functions. The model is varied and the process repeated until the total mean square difference over the four frequency/receiver depth combinations is a minimum. Rajan et al. [37] apply perturbation techniques to the problem using the eigenvalues obtained via the Hankel transform of the pressure field as the input.

In constructing the geoacoustic model with data from this experiment, information on water column properties will be available from separate measurements to provide the sound speed value needed to transform the index of refraction output into a sound speed profile.

\subsection{Bottom Models and Inversions with Synthetic Data}

We will use two model waveguides each having a one hundred meter deep isovelocity water column, a one hundred meter thick sediment layer, and a basalt basement (half-space) having density, compressional wave speed, and attenuation equal to nominal values for basalt (shear 
properties are not included in the models). Two models will be used for the sediment layer: a model with a terrigenous sediment layer made up of silty sand, and model consisting of fine sand sediment. The data on which both models are based is contained in Hamilton's 1980 review article on geoacoustic models [25]. For both bottom types the sediment density has been taken as constant due to constraints in SNAP, the acoustic modelling program which was used to calculate the horizontal wavenumbers for the sound field in the waveguides [27].

\subsubsection{The Terrigenous Bottom Model}

The constituents of terrigenous sediments are derived from weathering and erosion of rocks found mostly on land [40]. Hamilton obtained the following regression equation for velocity as a function of the depth in the sediments using data assembled for twenty areas in upper, unlithified layers of mostly turbidites in various oceans [25]

$$
V(z)=1.511+1.301 z-0.741 z^{2}+0.257 z^{3}
$$

The depth $z$ is in kilometers, and the velocity $V$ is in kilometers per second. The density in the sediment layers has been taken as $\rho=1.0 \mathrm{~g} / \mathrm{cm}^{3}$ so that the eigenvalues will be governed solely by the sound speed profile and the boundary conditions at the ocean surface and the sediment/basement interface. Water column sound speed has been set equal to the sediment sound speed at the water-sediment interface for the same reason. Figure (4-2) is the sound speed profile for the waveguide with a terrigenous bottom model.

SNAP produced eigenvalues for 53 propagating modes in this waveguide; however, only the first 15 of these eigenvalues were used in the inversion in order to avoid effects arising from interactions of the modes with the basement half-space since we are interested in testing our ability to recover the sediment sound speed profile. The highest mode used turned at a depth of 176 meters. The modes were treated as one turning point modes, and both inversion methods were used. Figures (4-3) through (4-6) show the inversion results with the errors plotted as depth error versus sound speed. As expected, the largest errors occur at the water-sediment interface where the extrapolation from the data point corresponding to the first mode to the point at $\xi=1, F(\xi)=0$ is made and where the profile does not meet the smoothness assumptions. We have also ignored the possibility of reflections at the interface which may introduce errors in $F(\xi)$ for the low order modes (those with large equivalent angles 
of incidence). The apparent increase in error at the lower depths reached by the inversion is believed to result from inaccuracies in the spline fit near the end of the data. Note that the inversion does not produce any points on the isovelocity portion of the profile since there are no turning points in an isovelocity segment (the one inversion point shown at the water surface is the reference sound speed $i$. e. the assumed minimum in the profile). Terrigenous sediments are predicted to have a sound speed at the water/sediment interface lower than the water sound speed [25]. The inversion methods cannot distinguish such a low velocity zone because they are finding turning depths which result from increasing sound speeds. In figure (4-7) are the results for model 1 with the water column sound speed increased to 1545 meters per second to give a low velocity zone. The reference sound speed used in the inversion was taken as 1545 meters per second which, because of the normalization of the eigenvalues, produced a value of $\xi>1$ for the first eigenvalue and, to produce the results of figure (4-7), the first eigenvalue was discarded. Thus the data give a clear indication that a grossly inaccurate reference sound speed has been used. If the first eigenvalue is retained and the correct reference sound speed of 1511 meters per second used, then the result obtained is shown in figure (4-8). These results indicate that the inverse methods will at least indicate the presence of a low velocity zone at the water/sediment interface, and it may be possible to gain some information about the value of the minimum sound speed through iterative adjustments to the reference sound speed. 


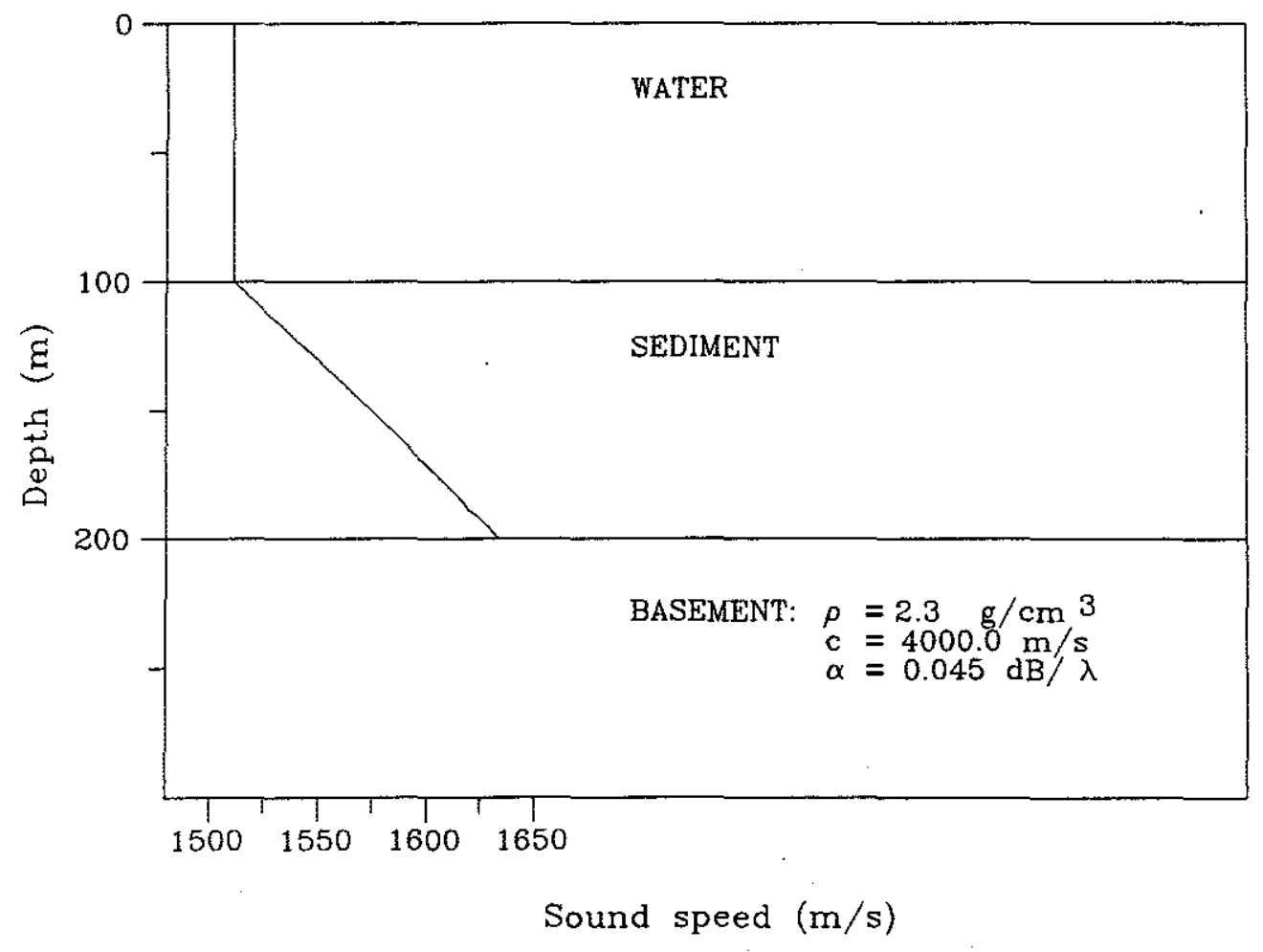

Figure 4-2: The shallow water waveguide model with a terrigenous sediment bottom. 


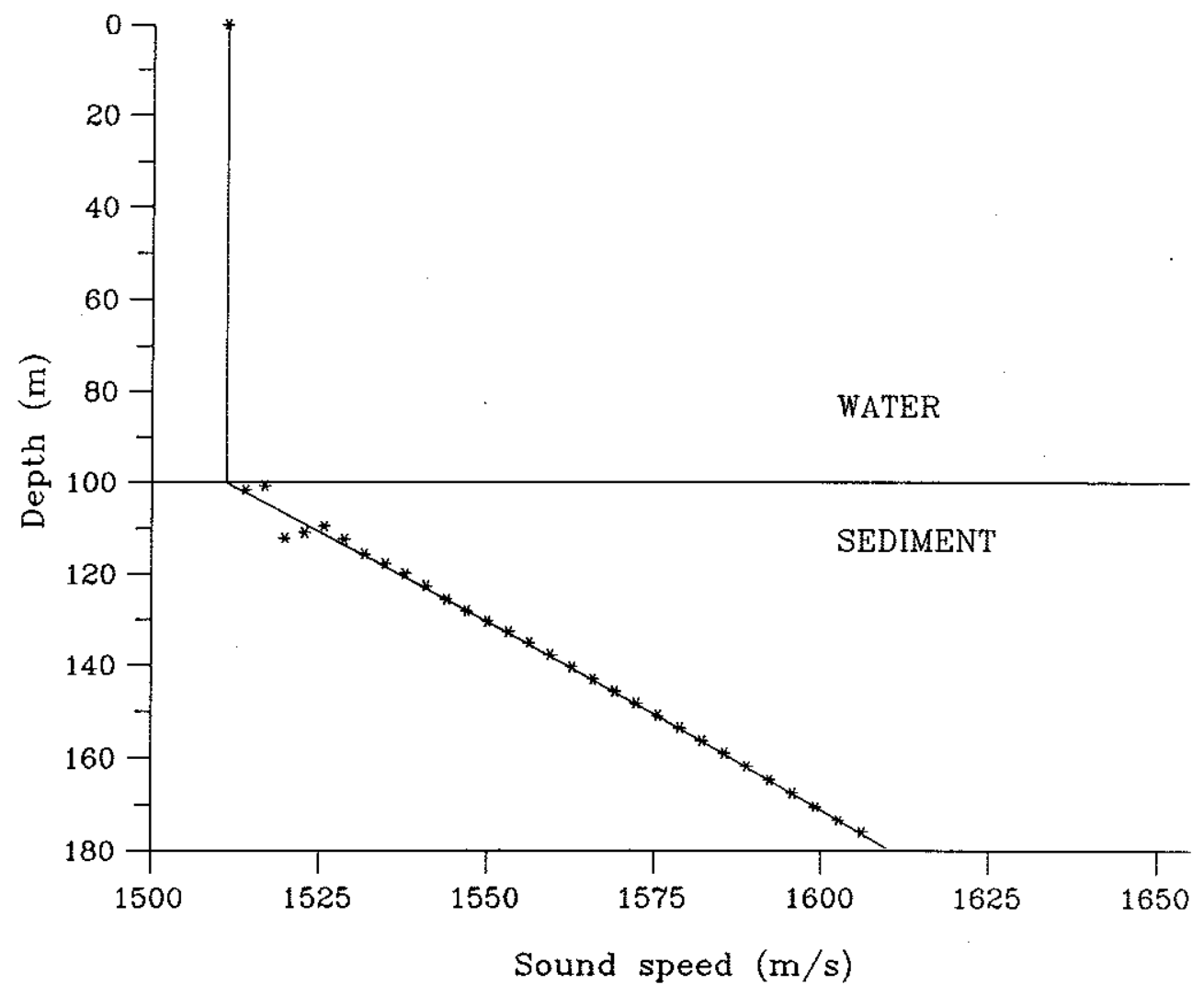

Figure 4-3: Inversion results for waveguide model 1 using inversion method 1 . The background curve is the actual sound speed profile in the waveguide. 


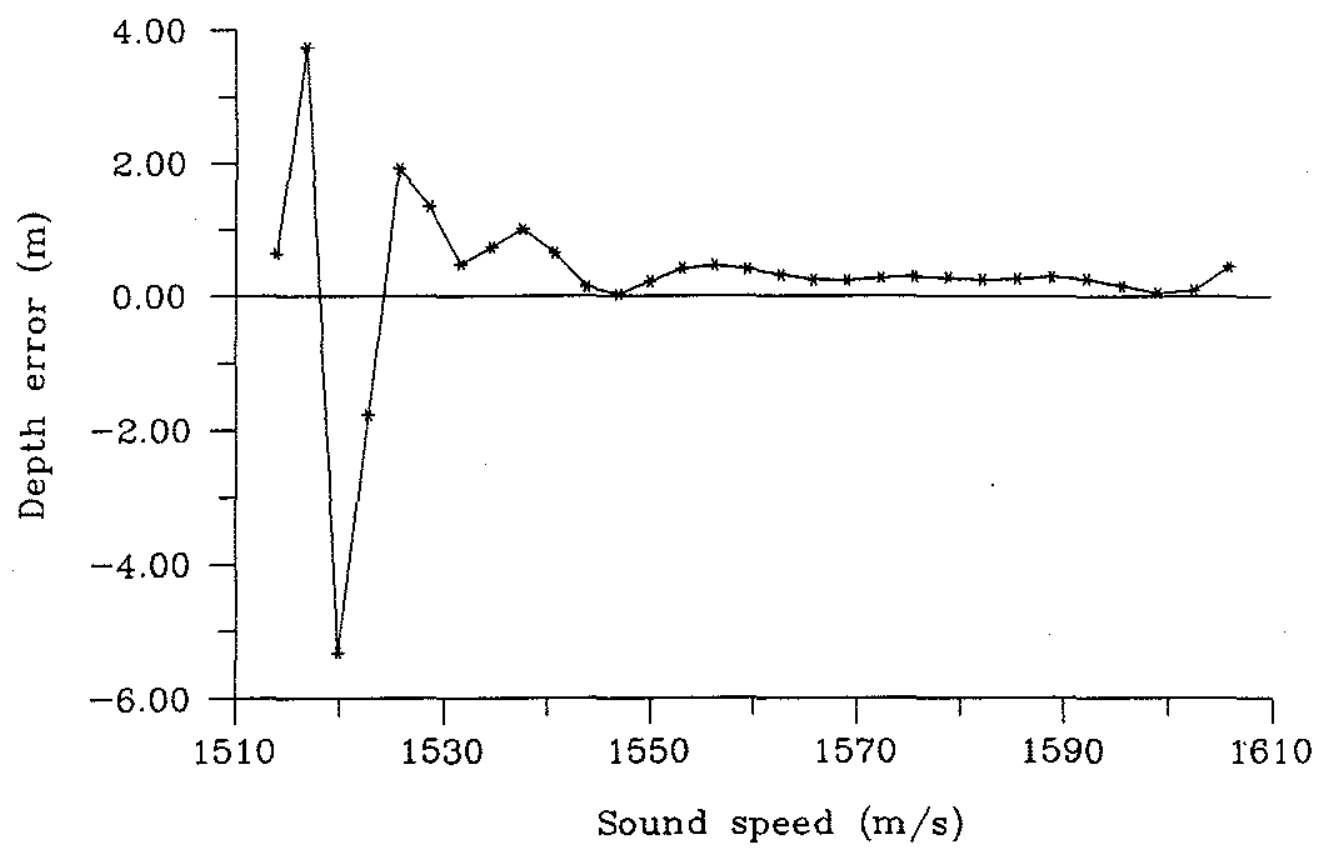

Figure 4-4: Depth error for inversion method 1 applied to waveguide model 1.

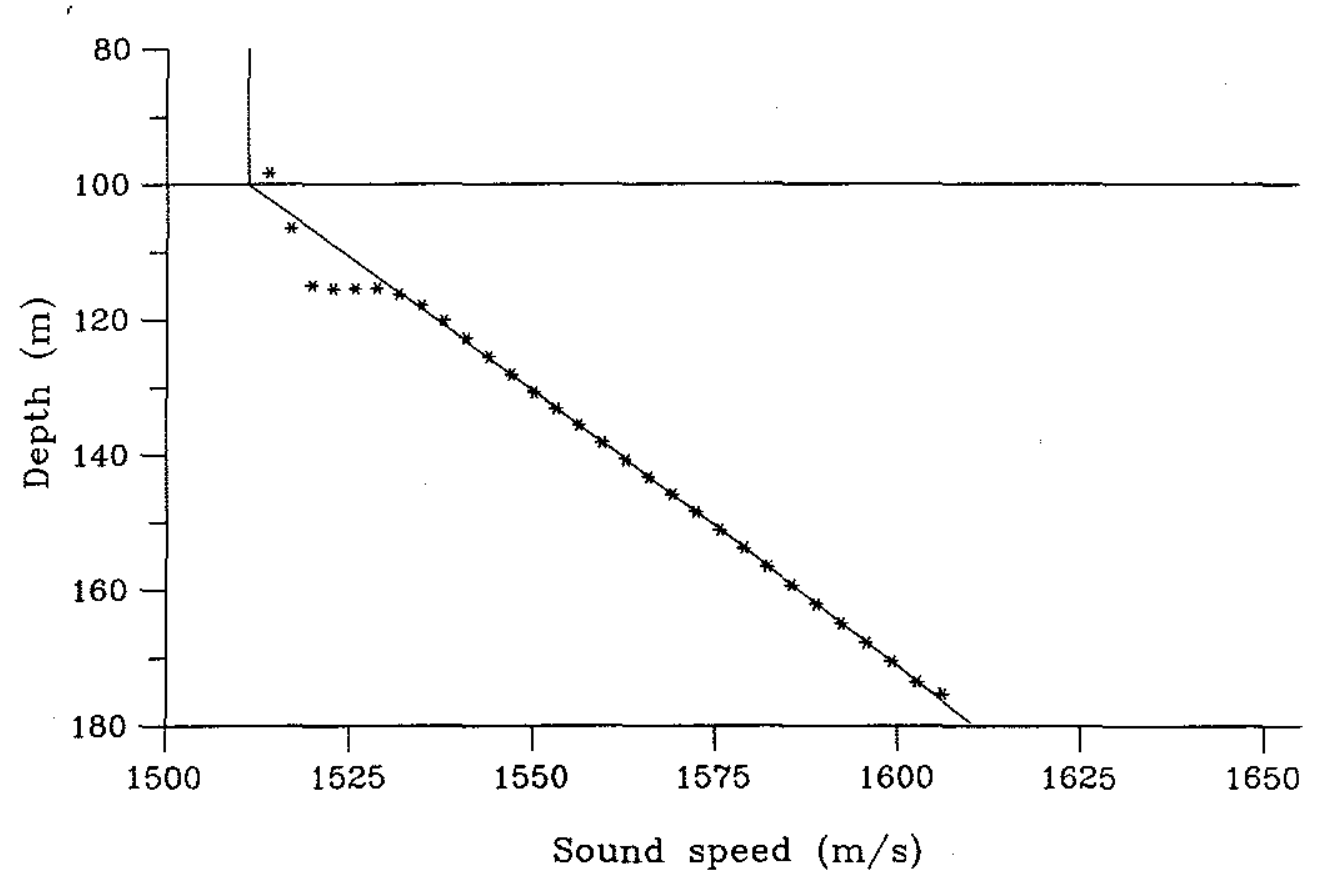

Figure 4-5: Inversion results for waveguide model 1 using inversion method 2. The background curve is the actual sound speed profile in the waveguide. 


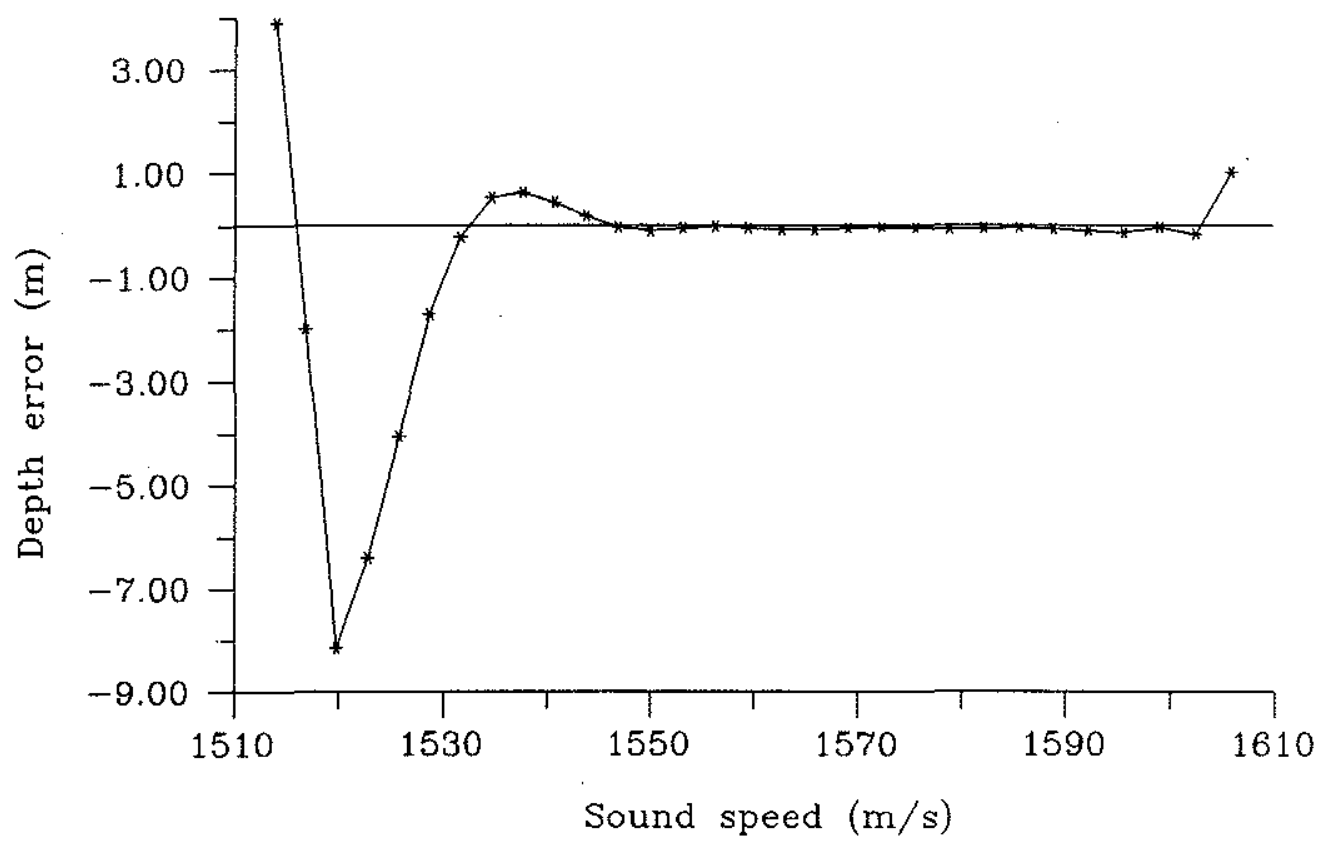

Figure 4-6: Depth error for inversion method 2 applied to waveguide model 1. 


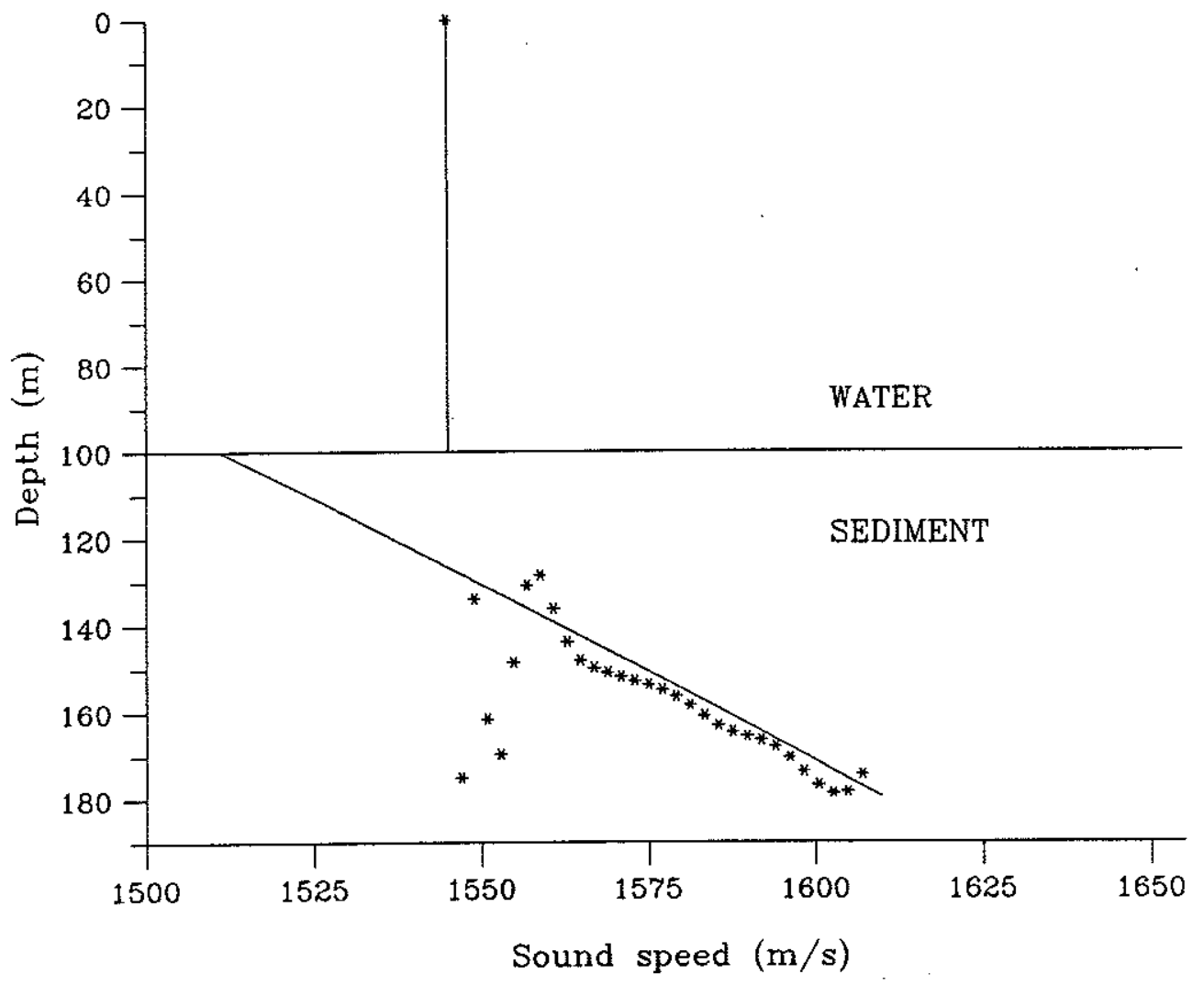

Figure 4-7: Inversion results for a terrigenous waveguide with a low velocity zone at the water/sediment interface. The eigenvalue of the lowest mode was discarded since it produced a value of $\xi>1$. 


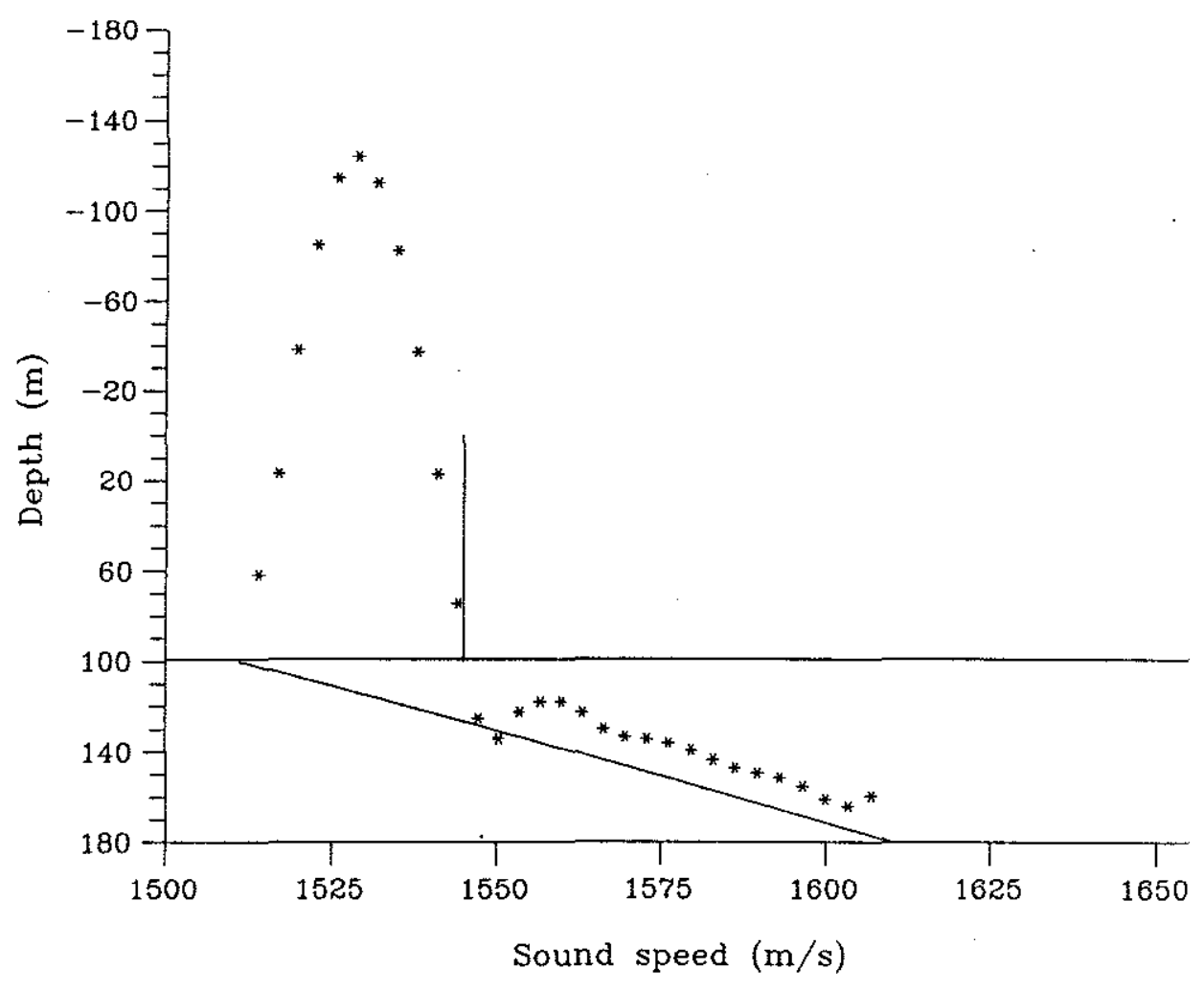

Figure 4-8: Inversion results for a terrigenous waveguide with a low velocity zone at the water/sediment interface obtained using the correct reference sound speed of 1511 meters per second. 


\subsubsection{The Fine Sand Bottom}

The fine sand bottom has a large discontinuity in the compressional wave speed at the water/sediment interface and large sound speed gradients in the top portion of the sediment layer. A regression equation given by Hamilton for the sound speed in the first twenty meters of a fine sand bottom is

$$
V(z)=1806.0 z^{0.015}
$$

The depth $z$ is in meters and the resulting sound speed $V$ is in meters per second. For depths greater than twenty meters, the profile has been extended as a straight line with slope $1.4168 \mathrm{~s}^{-1}$ which is the sound speed gradient of equation (4.2) at a depth of twenty meters. Figure (4-9) is the sound speed profile for the fine sand bottom model.

For this model SNAP generated eigenvalues for 47 propagating modes of which 23 were used for the inversions (the turning depth of the highest mode used was 174 meters). Both inversion methods were again used for the inversions, and, in addition, both the least squares and the smoothing spline routines were used. The results are shown in figures (4-10) through (4-17). An attempt was made to treat the modes with equivalent incidence angles greater than the critical angle as interacting with a critically reflecting bottom which was modeled as a 1727 meter per second half-space (i.e. they were treated as case 3 modes); however, this made the agreement between the inversion results and the actual profile worse than for previous inversions which treated all the modes as simple one turning point modes (figure $(4-18))$.

Finally, we used all the eigenvalues in the inversion process for this model (figure (4-19)). The inversion output sound speed approaches the basement sound speed value while the depth value does not depart significantly from the sediment/basement interface depth. 


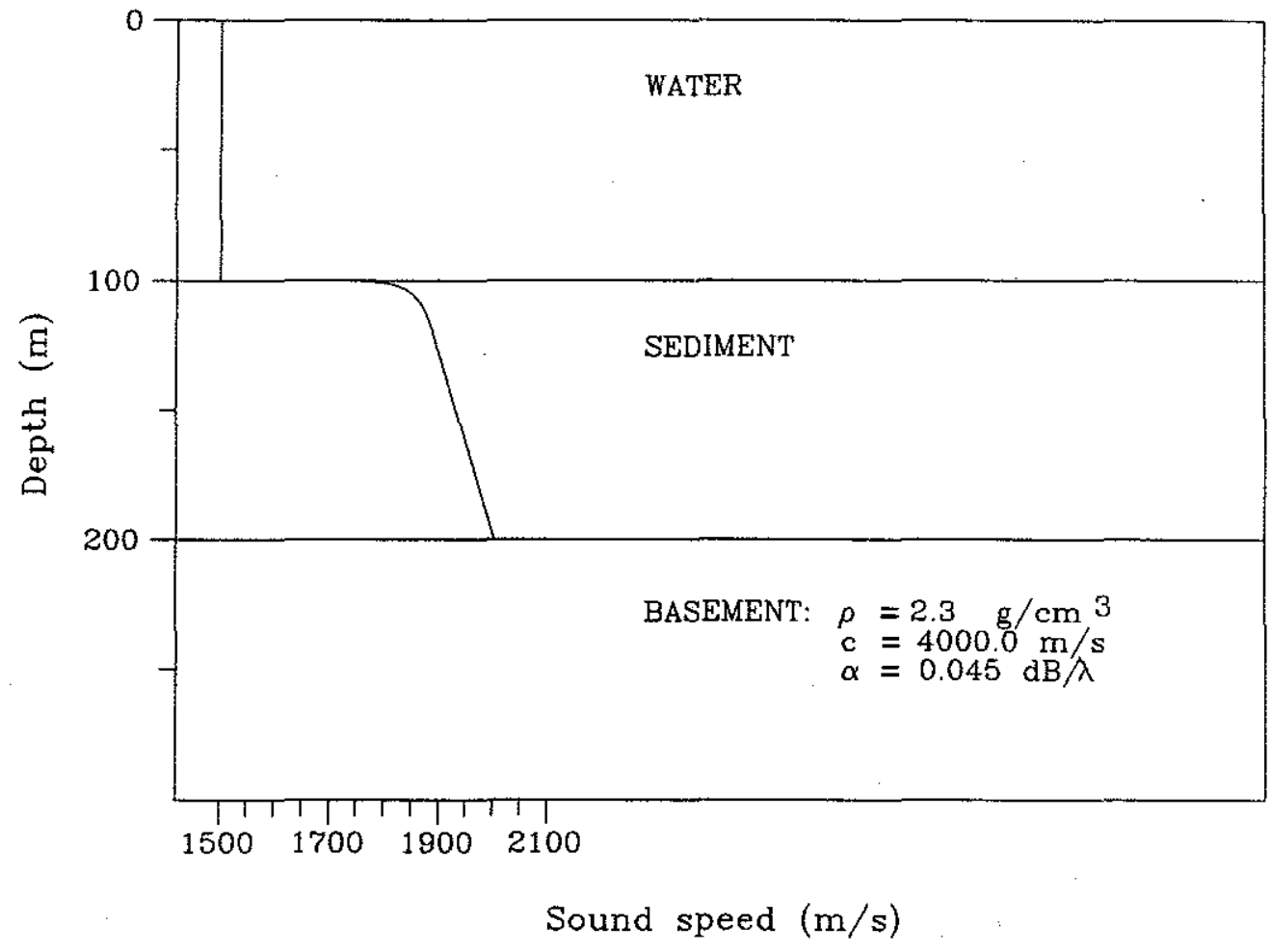

Figure 4-9: The shallow water waveguide model with a fine sand bottom. 


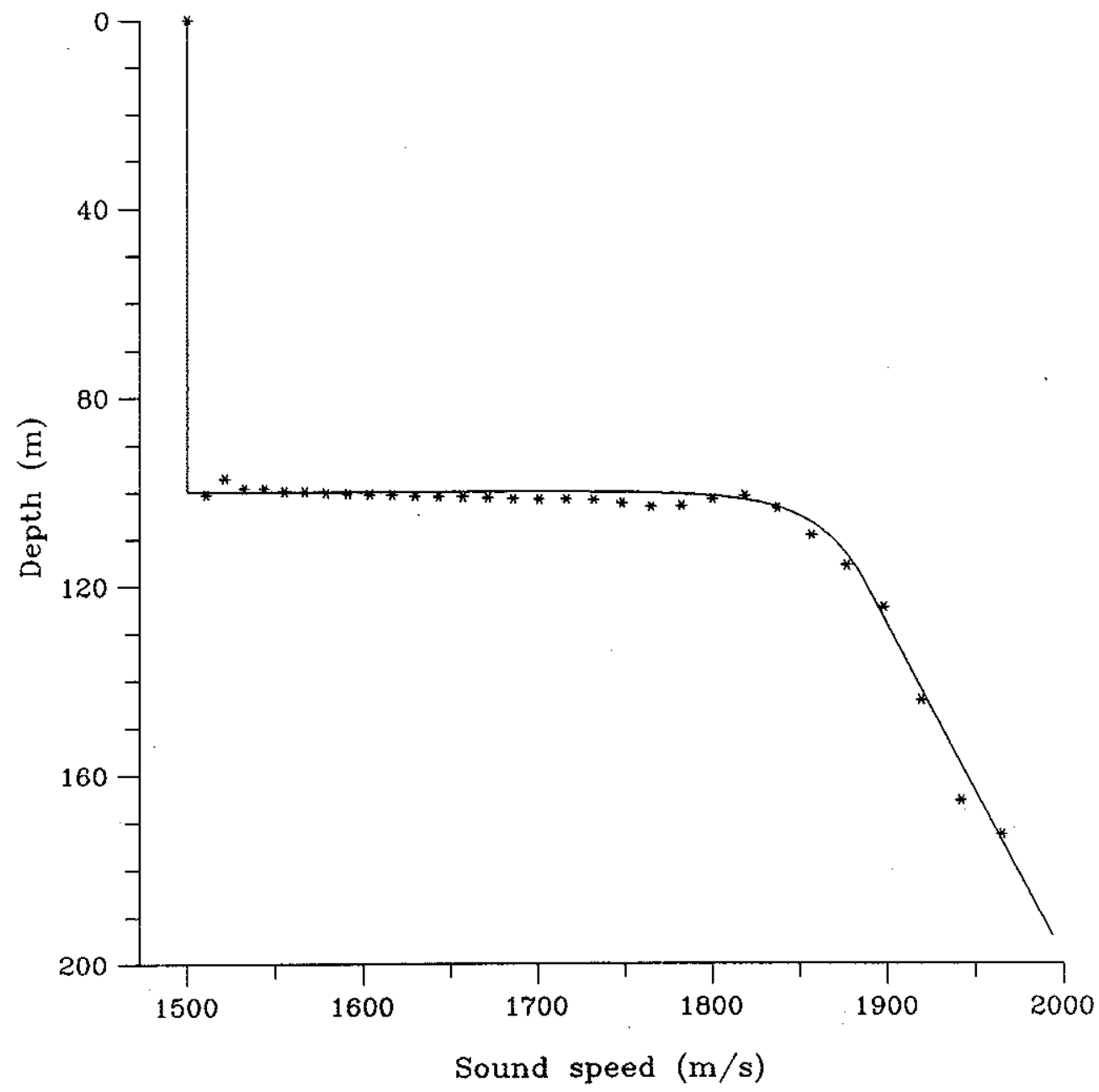

Figure 4-10: Inversion results for waveguide model 2 using inversion method 1 (least squares splines). 


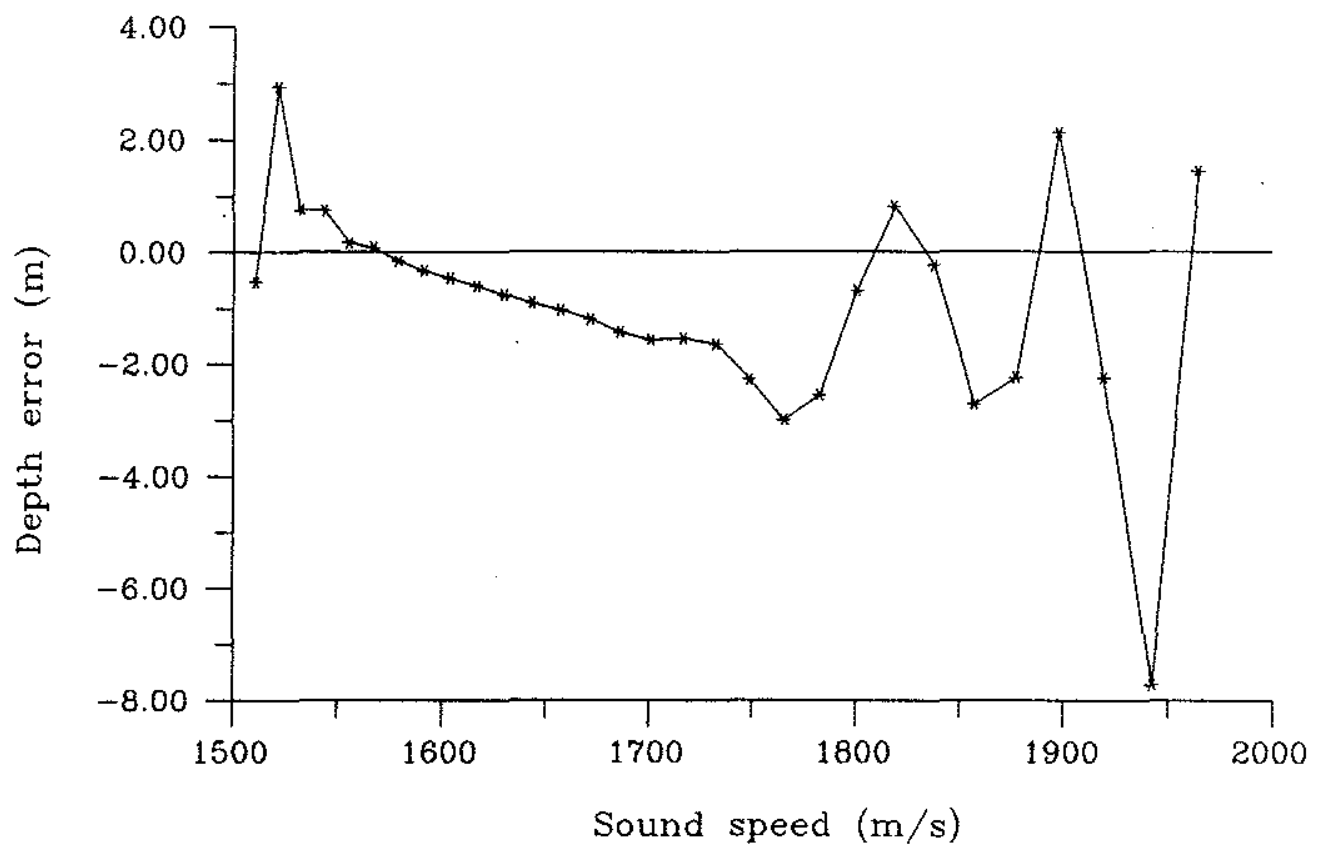

Figure 4-11: Depth error for inversion method 1 (least squares splines) applied to waveguide model 2. 


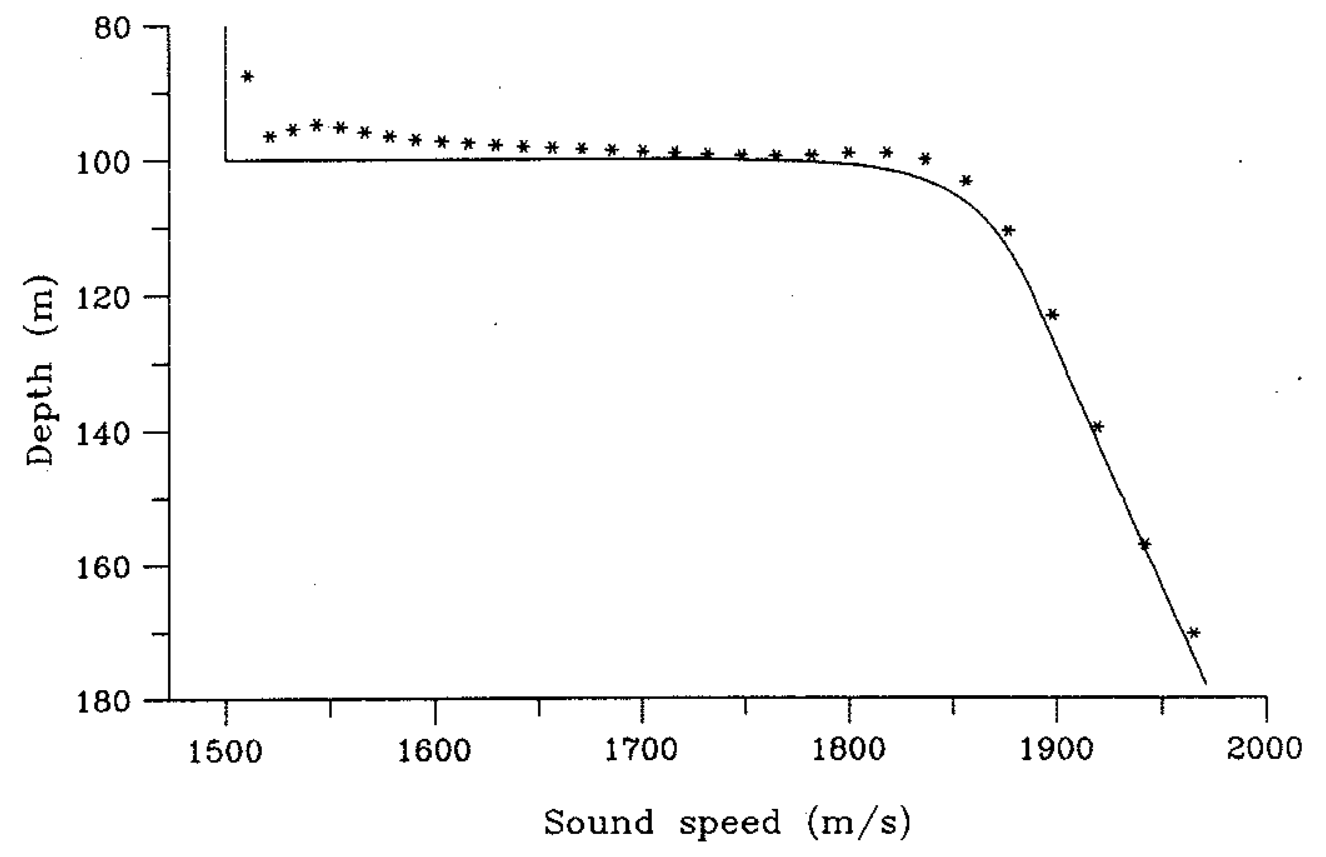

Figure 4-12: Inversion results for waveguide model 2 using inversion method 1 (smoothing splines). 


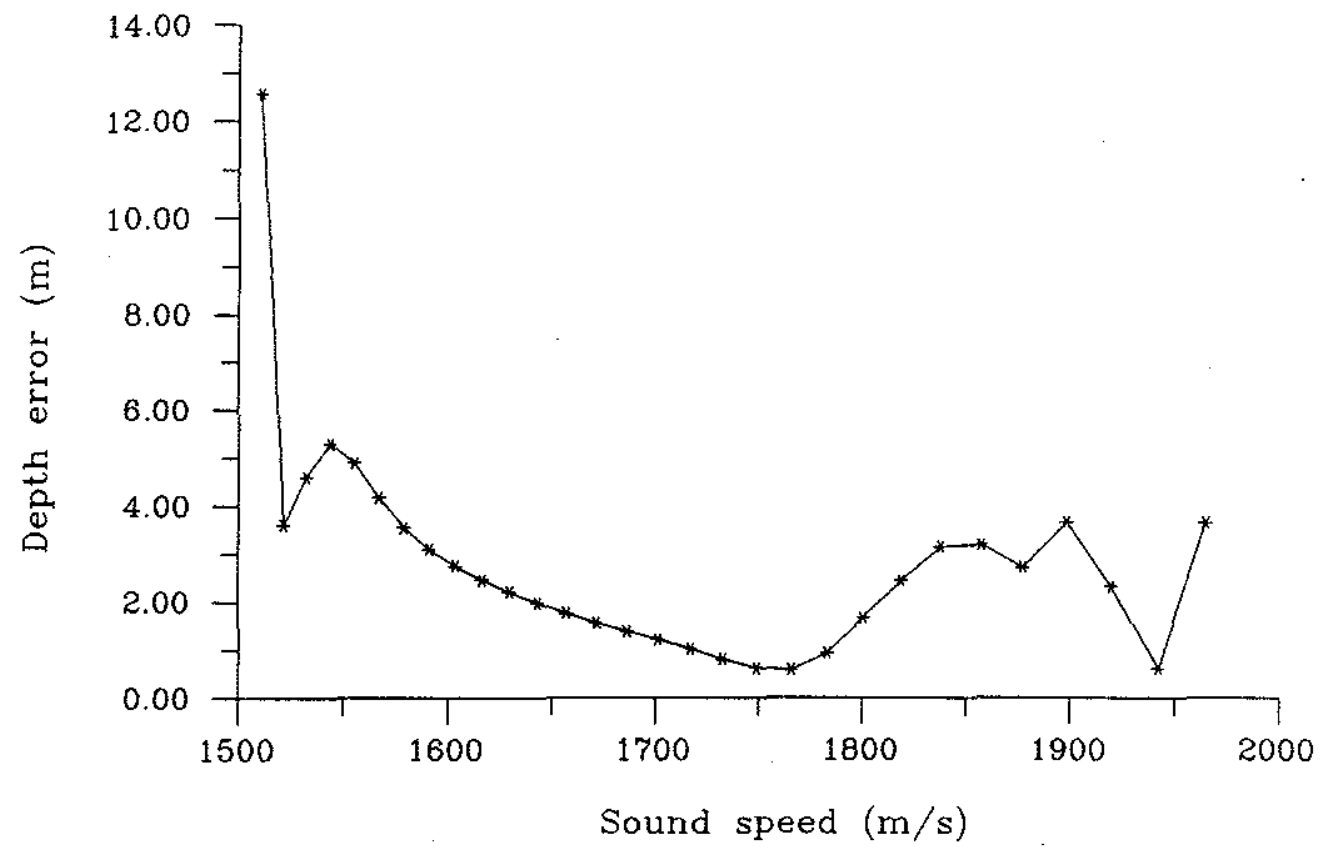

Figure 4-13: Depth error for inversion method 1 (smoothing splines) applied to waveguide model 2. 


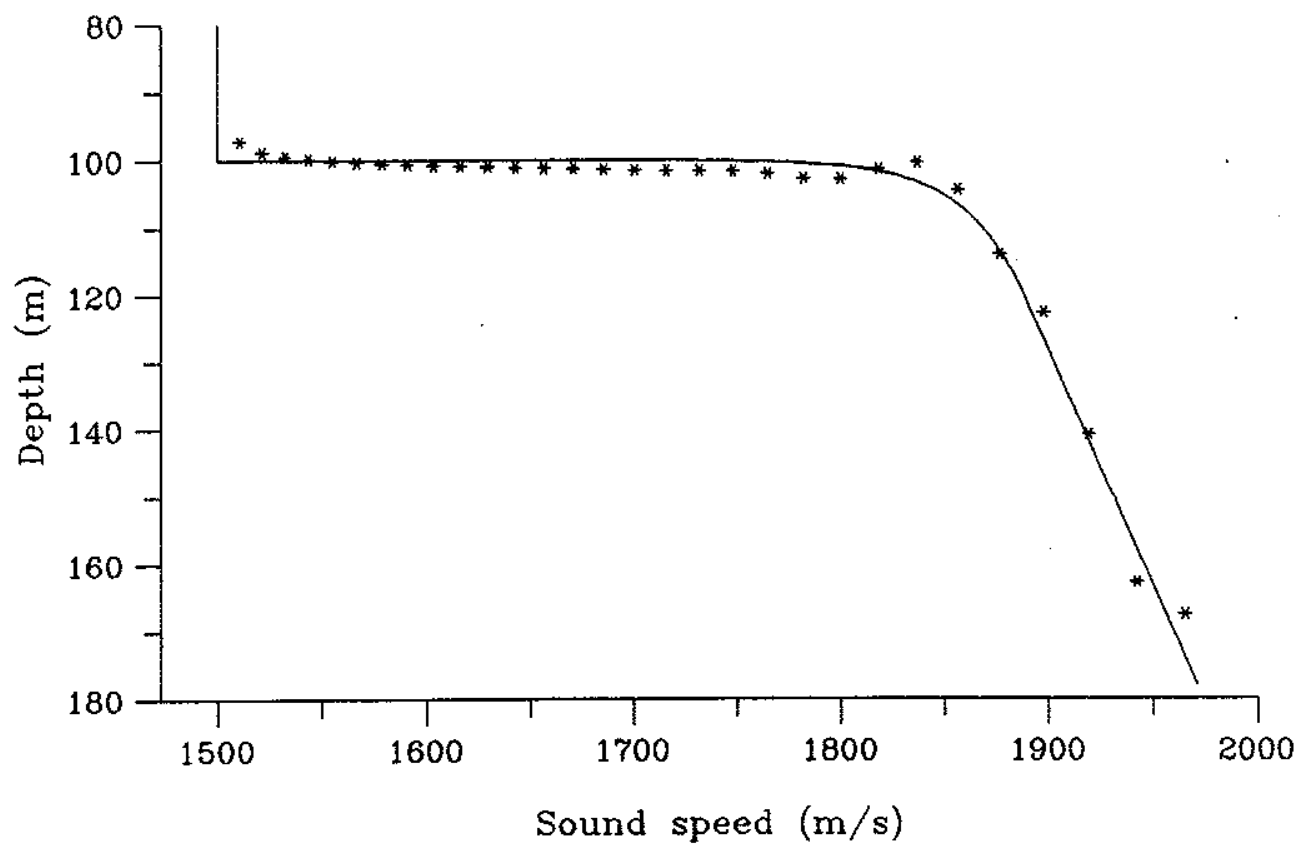

Figure 4-14: Inversion results for waveguide model 2 using inversion method 2 (least squares splines). 


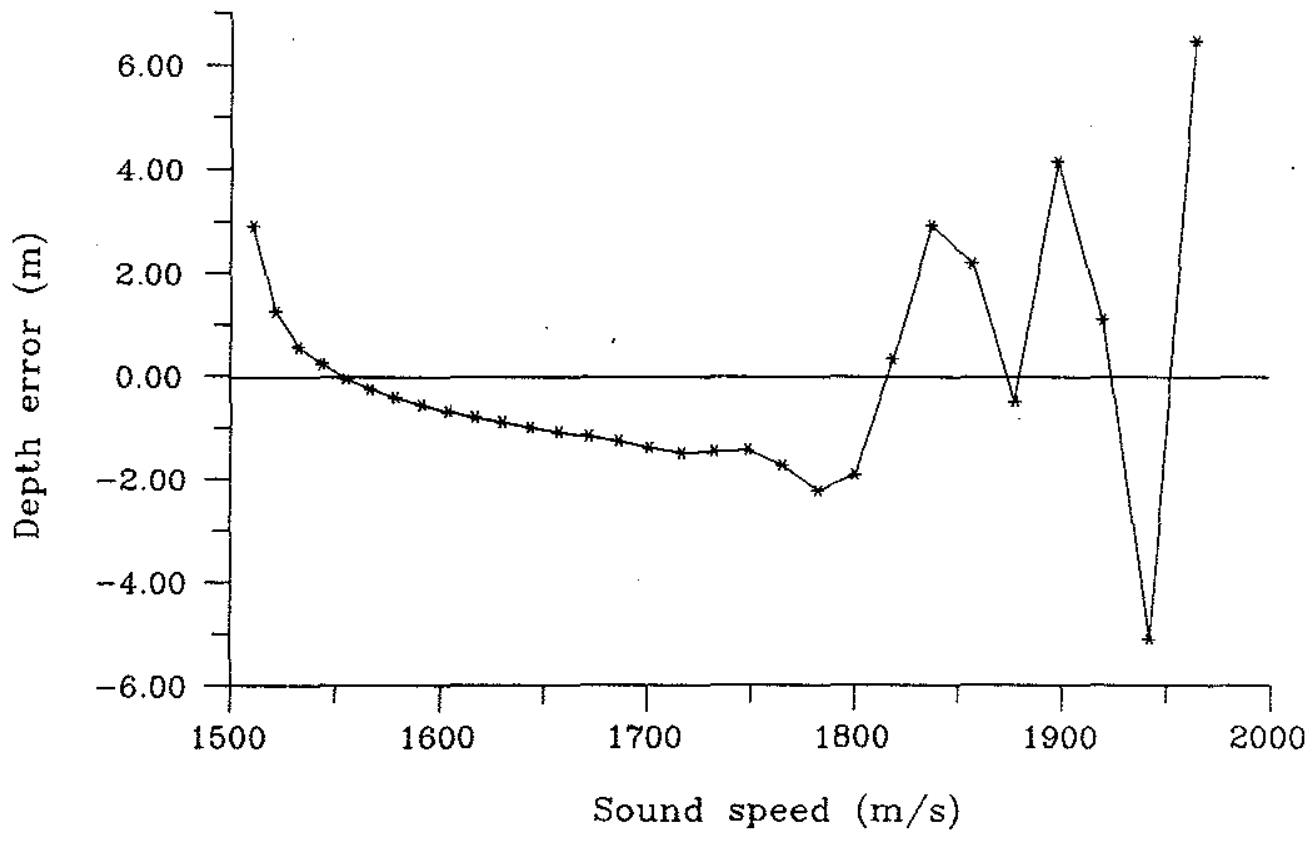

Figure 4-15: Depth error for inversion method 2 (least squares splines) applied to waveguide model 2. 


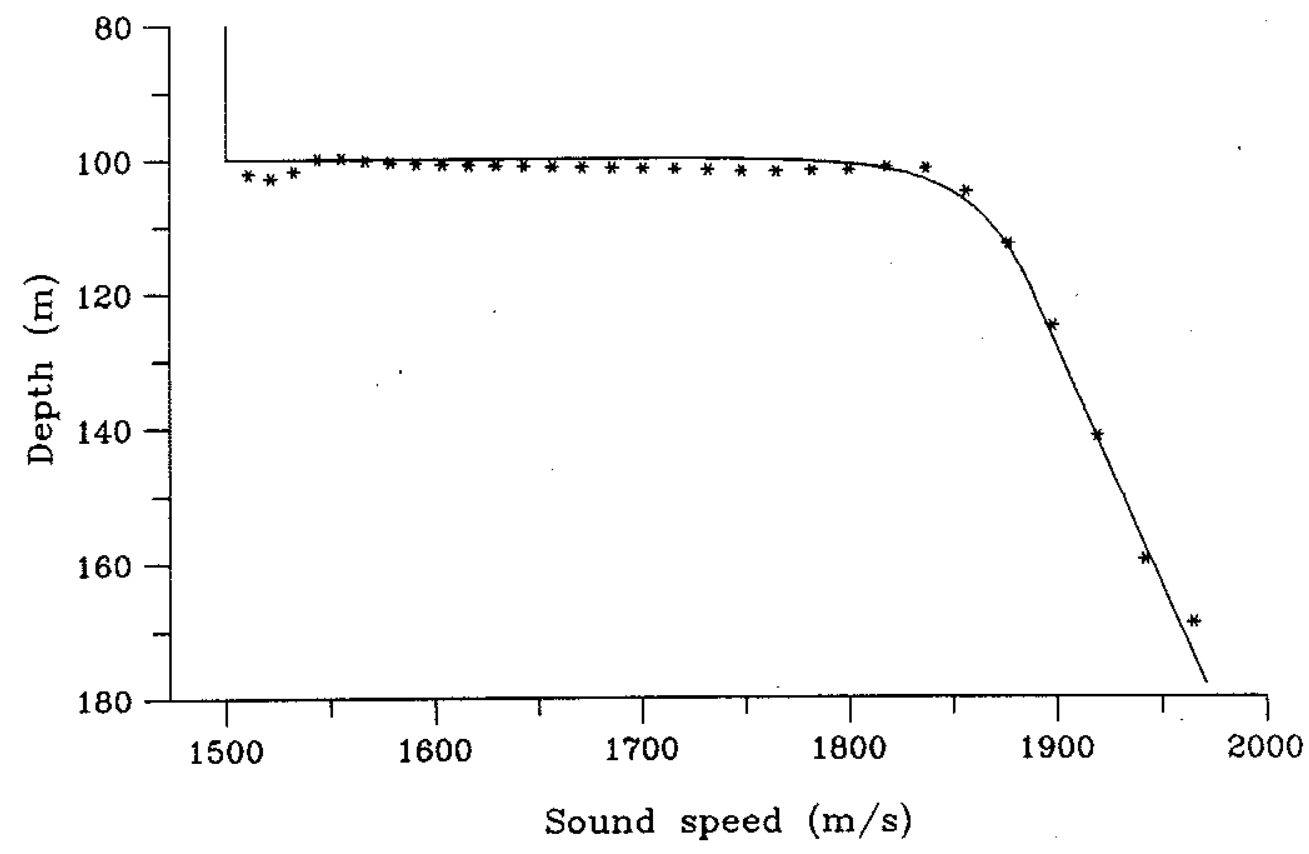

Figure 4-16: Inversion results for waveguide model 2 using inversion method 2 (smoothing splines). 


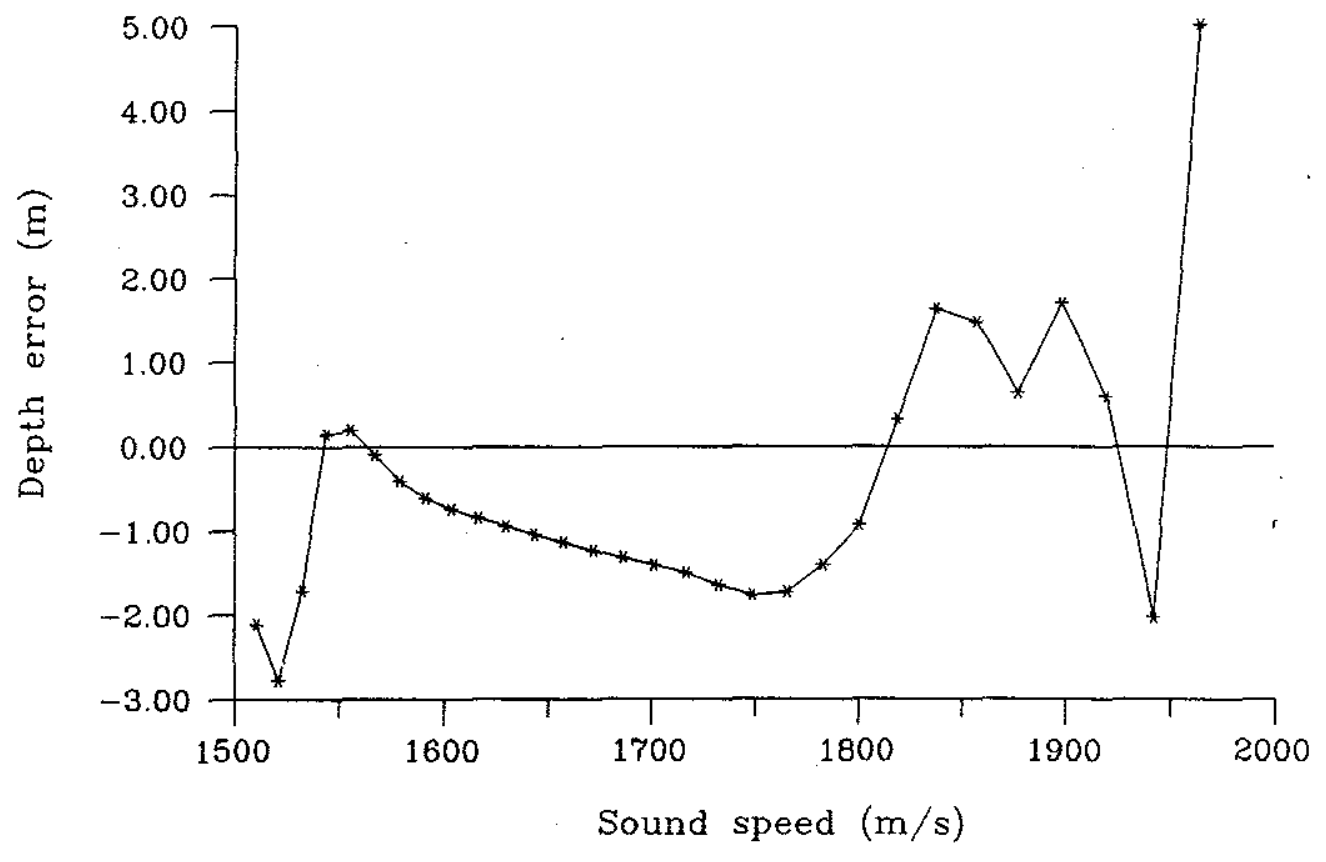

Figure 4-17: Depth error for inversion method 2 (smoothing splines) applied to waveguide model 2. 


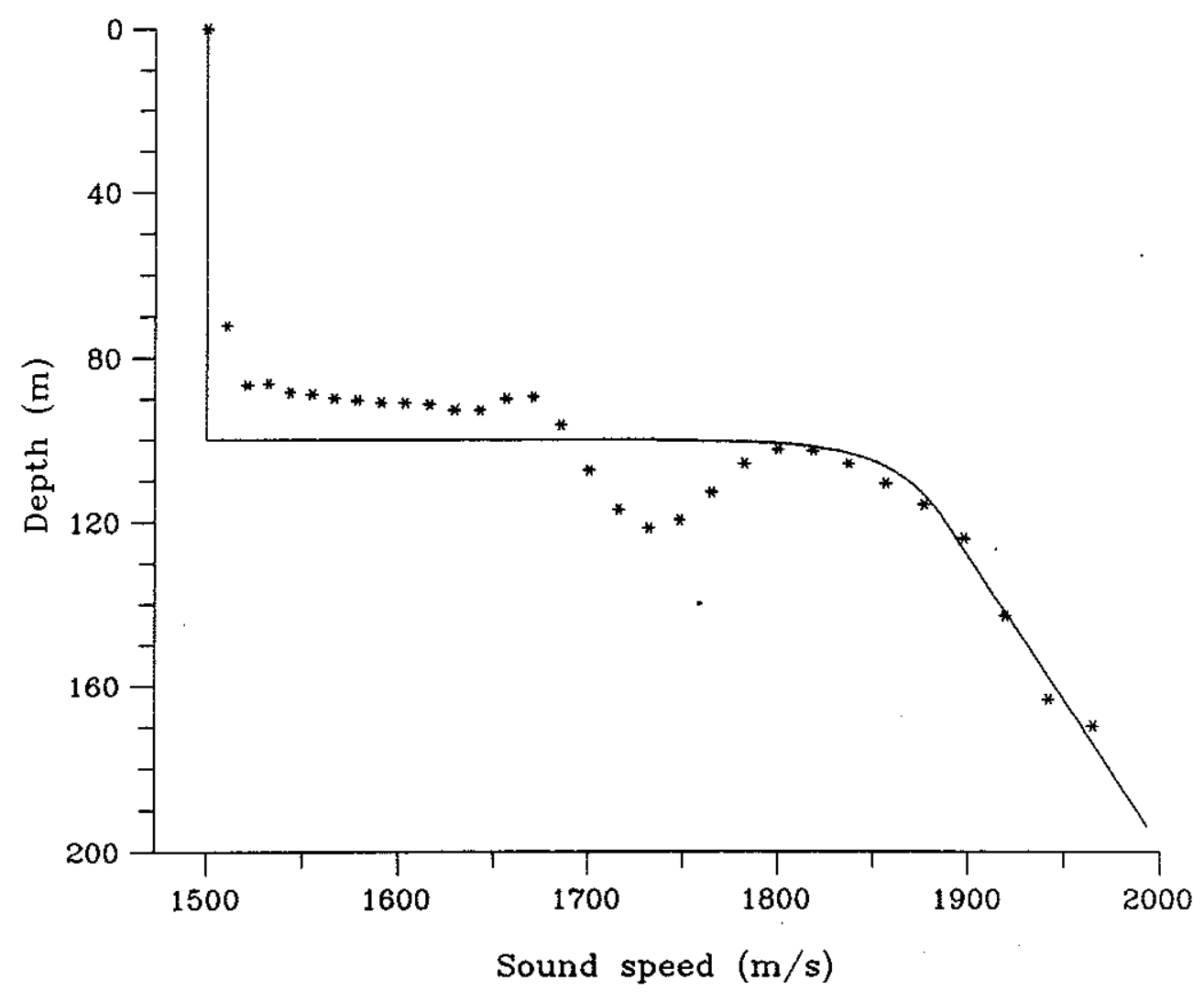

Figure 4-18: Inversion results for waveguide model 2 using inversion method 1 (least squares splines) treating the low order modes as reflecting from a half-space with a 1727 meter per second sound speed. 


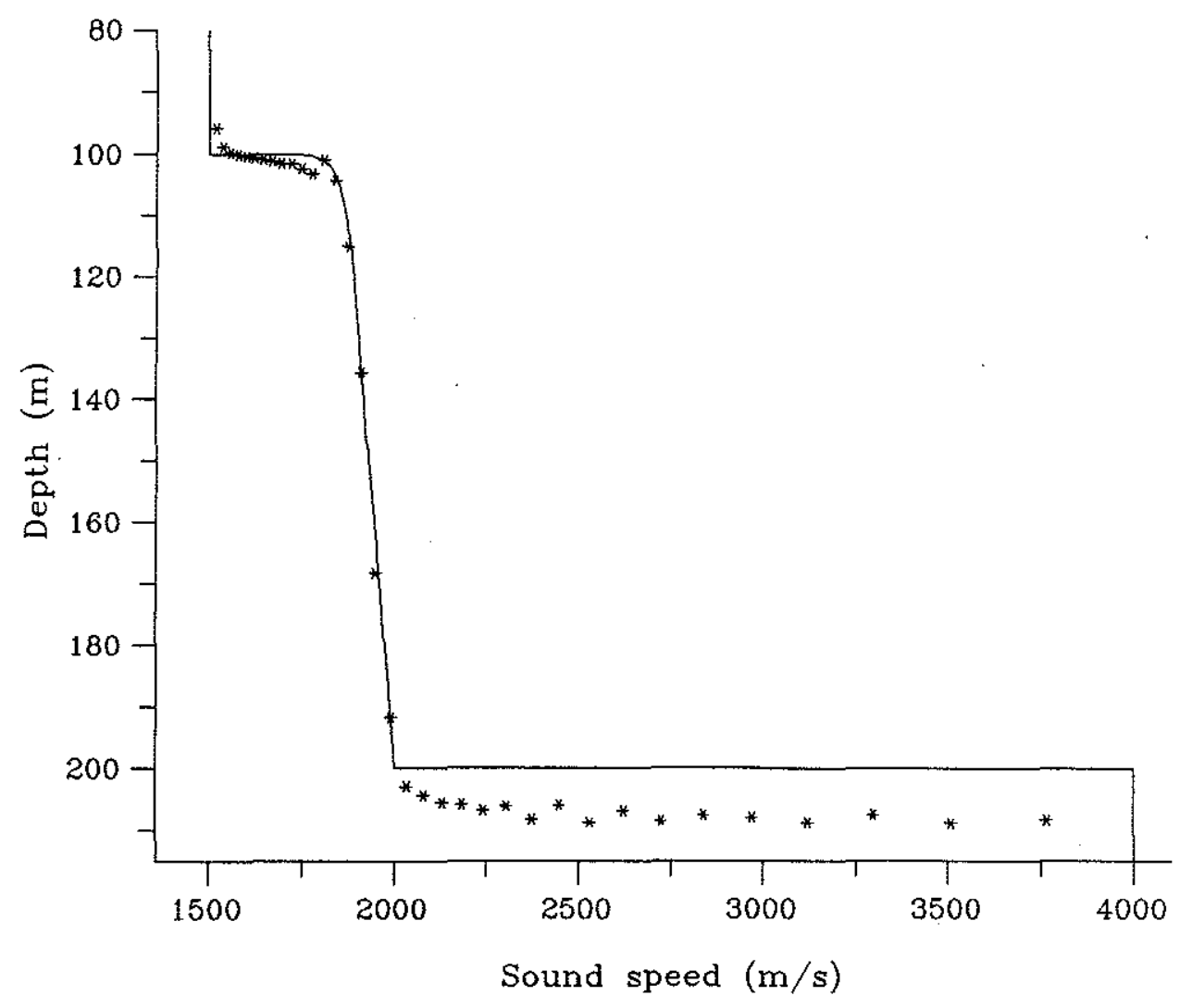

Figure 4-19: Inversion results for waveguide model 2 using inversion method 1 (least squares splines) using the eigenvalues of all propagating modes. 


\subsection{Experimental Results}

An experiment was performed in the Gulf of Mexico near Corpus Christi, Texas during September, 1985 using a configuration similar to that of Figure (4.1). For the Corpus Christi site the water depth was 30.3 meters, source depth was 22.86 meters, and the two receivers were 1.5 meters and 15.0 meters above the bottom (G. V. Frisk pers. com. 1988) Geologic data [31] for the region predicts a 17.5 meter thick layer of silty clay with an estimated sound speed at the water/sediment interface of 1517.6 meters per second increasing to 1531.5 meters per second at the layer bottom. Since the sound speed in the water column was 1545 meters per second, this gives a low velocity zone. Beneath the silty clay layer, a layer of very fine sand was predicted to extend to 45 meters with a sound speed of 1737.1 meters per second at the interface between the layers and increasing to 1763.0 meters per second at the bottom of the layer. In the absence of better information, parameters for this segment were extended to a depth of 100 meters. It should be emphasized that these values are based on a study of the geology of the region, and the sound speed predictions are based on the type of sediments and the estimates of Hamilton's work for such sediment types [25]. Figure (4-20) is the predicted sound speed profile in the sediments. The eigenvalues were obtained by S. D. Rajan (pers. com. 1986) from the depth dependent Green's function of the measured sound field via the numerical Hankel transform, and are presented in Table (4.1). Water column sound speed at

\begin{tabular}{ccc} 
Frequency $(\mathrm{Hz})$ & Mode Number & Eigenvalue $\left(\mathrm{m}^{-1}\right)$ \\
\hline 50.0 & 1 & 0.1972886 \\
& 2 & 0.182872 \\
140.05 & 1 & 0.5678598 \\
& 2 & 0.5572134 \\
& 3 & 0.5440619 \\
& 4 & 0.5252742 \\
& 5 & 0.5102440
\end{tabular}

Table 4.1: Experimentally measured eigenvalues for the Corpus Christi site.

the experiment site was 1545 meters per second. Since a total of only seven eigenvalues at the two frequencies is available, we combined the data to form a single data set which is possible 


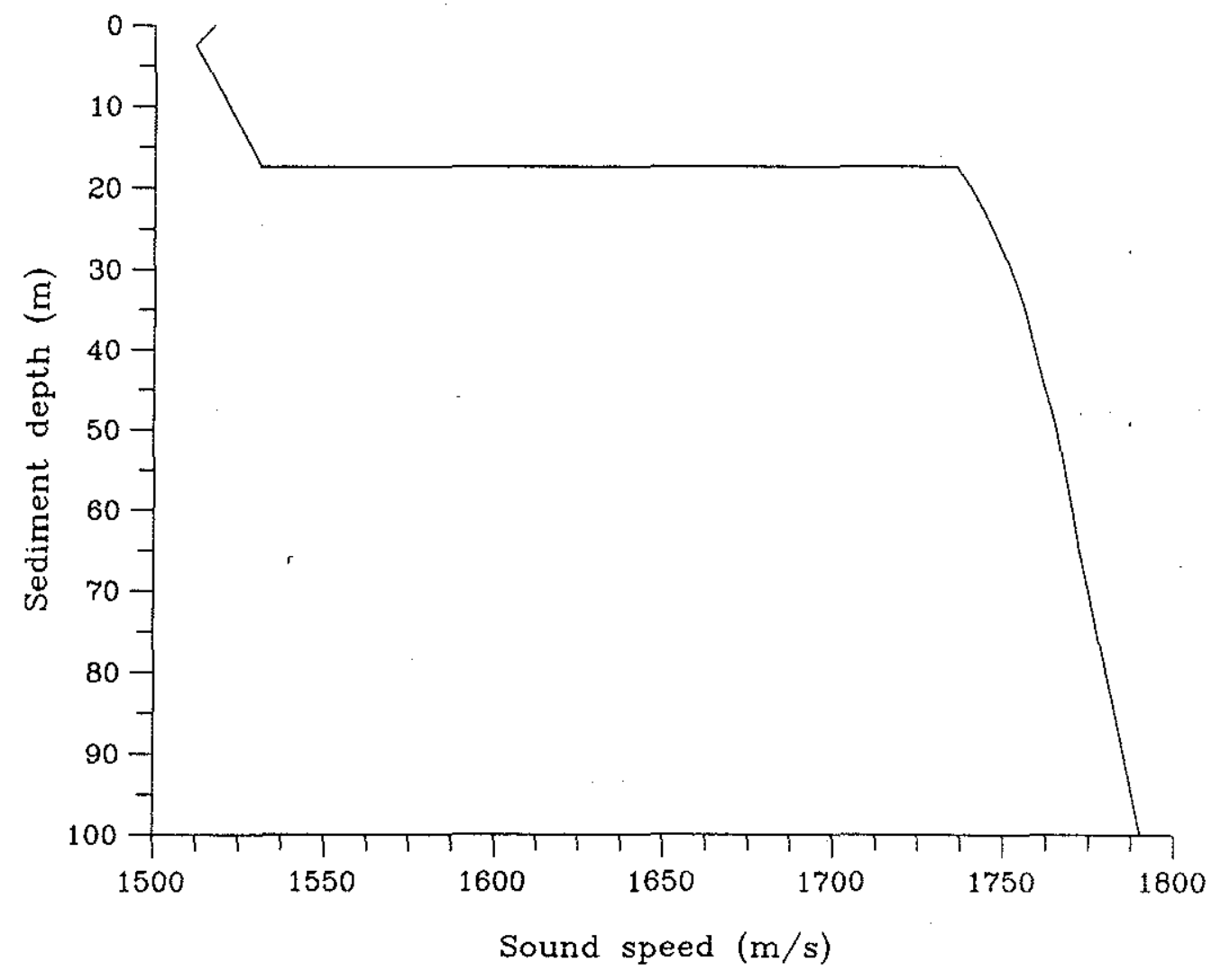

Figure 4-20: Sound speed profile for the Corpus Christi area based on geologic information. 


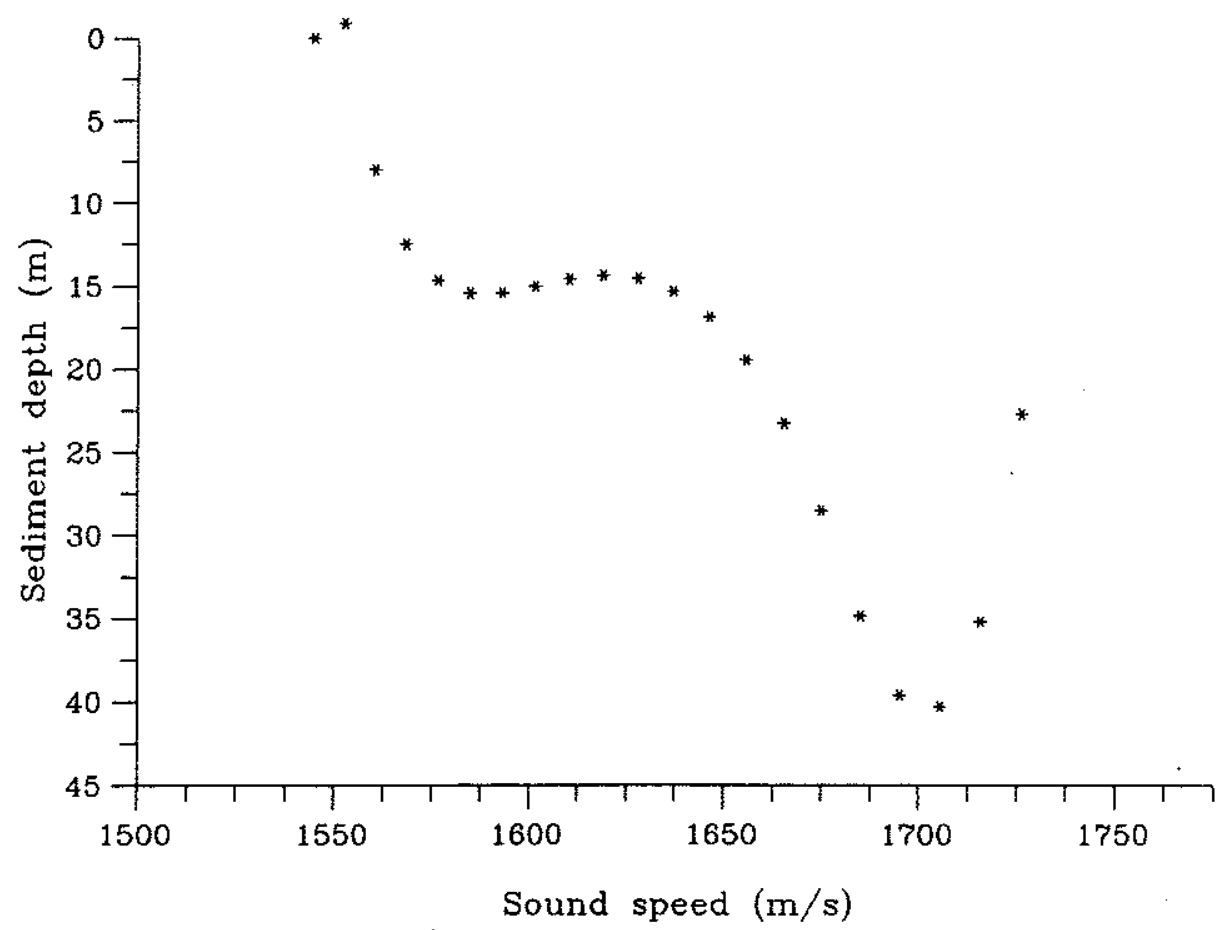

Figure 4-21: Corpus Christi area sediment sound speed profile obtained using all seven eigenvalues at $50 \mathrm{~Hz}$ and $140.05 \mathrm{~Hz}$.

since $F(\xi)$ as we have defined it is frequency independent. The results of the inversion using method 1 with an inversion program using the least squares spline routines of the PORT Library [18] are shown in figure (4-21). (The PORT Library program was used because it seemed to handle the small data set better than the program using the IMSL routines.) The large oscillation at the bottom of the inversion results suggets a data error at the end of the data set (smallest $\xi$ ). After $\xi$ is calculated, the second $50 \mathrm{~Hz}$ eigenvalue is the last data point, and if this eigenvalue is removed, the oscillation is also removed as shown in figure (4-22)) which also shows the results of a perturbative inversion done by S. D. Rajan (pers. com. 1986), and the predicted sound speed profile. 


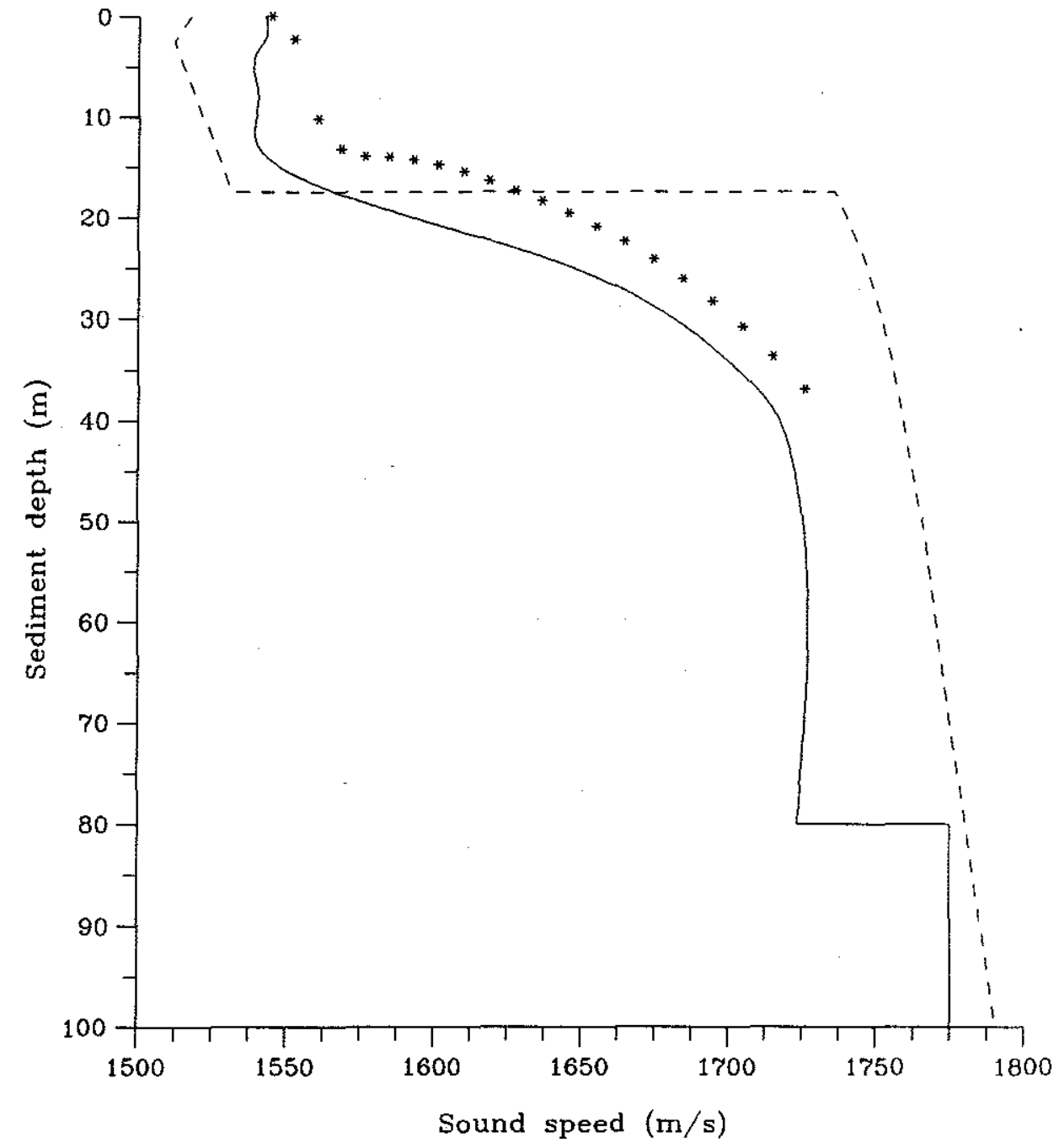

Figure 4-22: Corpus Christi sediment sound speed profile obtained with inversion method 1 after removing the second $50 \mathrm{~Hz}$ eigenvalue, the profile obtained perturbatively by Rajan (solid curve), and predicted profile (dotted curve). 


\section{Chapter 5}

\section{Conclusions}

\subsection{Summary}

In this thesis we have shown that using a WKB inversion technique based on the WKB phase integral equations and using modal eigenvalues as input data provides a method for obtaining information about index of refraction profiles in the ocean waveguide.Information can be obtained for the water column and for the sediment layers. Two approaches for transforming the WKB phase integral to a relation between depth and the index of refraction were developed. Both methods assume that the value of the WKB phase integral can be accurately estimated based on the number of turning points for a mode and/or the types of boundary interactions the mode experiences, and both methods rely on knowledge of at least the speed of sound at the surface.

Inversion method 1 differentiates the WKB phase integral equation to obtain an Abel integral equation which is then inverted to provide an equation for depth [43] and is only usable for monotonic or symmetric sound speed profiles. In the case of a symmetric profile the knowledge that the phase contributions above and below the axis are equal allow the original WKB phase integral to be divided into two equal portions which essentially puts the problem in monotonic form. With a value of sound speed at one depth, the monotonicity or symmetry allows conversion of the depth/index of refraction relation to a sound speed profile. For a general asymmetric profile, it is possible to to treat the data as though it was obtained from a symmetric profile and to obtain an 'equivalent symmetric profile' [35]. The utility of this approach remains to be seen.

Inversion method 2 uses fractional calculus methods [39] to find the inclusion (equation 
(3.28)) from the WKB phase integral. The inclusion is differentiated to obtain the depth difference between two points with the same index of refraction (the excursion). For a monotonic profile this gives the depth directly and for a symmetric profile the phase integral can be split into two equal parts as it was for inversion method 1 allowing determination of the full profile. For an asymmetric profile this method gives different answers in the portions of the profile with one and two turning points. In the portion with two turning points, the inversion result is the distance between two points of equal index of refraction, and in the single turning point case the result is the index of refraction. This assumes that we have at our disposal the sound speed at the surface (to allow the modes to be separated into the one and two turning point groups) and the minimum sound speed in the profile. The physical interpretation of the variable $\xi$ as $\sin ^{2} \theta$ which constrains it to the range $[0,1]$, suggests that it may also be possible to approximate the minimum sound speed value based on the range of the normalized eigenvalues. Since a known portion of the inversion result for a profile with both one and two turning point modes is the index of refraction as a function of depth, it may be possible to use an iterative procedure to arrive at a reasonable excursion versus sound speed curve and, perhaps, to separate the two branches of the sound velocity profile in the two turning point section, that is to find a good approximation to the entire sound speed profile.

Both methods have been implemented using splines for differentiation and integration. The techniques were applied to three idealized profiles to demonstrate the ability of the inversion methods to recover the profiles. A significant disadvantage of these methods is the present lack of quantitative statements concerning depth resolution and performance with inaccurate data. Performance of the methods with inaccurate data was demonstrated with numerical experiments using the $n^{2}(z)$ profile after adding random noise to the eigenvalues. The inversions are capable of giving results even with some noise in the data, and the use of inversion method 1 on a set of experimental data was demonstrated in Chapter 4 . The results of the inversion of the experimental data show that the inversion results may give a fairly clear indication ( large depth oscillations in the output) when an eigenvalue is incorrect. The depth resolution is related to the depth separation between succesive turning points; however, we do not yet have a quantitative expression for the resolution. 
In Chapter 4, both methods were applied to two models for a shallow water waveguide to illustrate the capability to provide estimates of sediment compressional wave speed profiles in shallow water. Finally, inversion method 1 was applied to a small data set from an experiment performed in the Gulf of Mexico. Only seven eigenvalues at two frequencies were available, and it appears that one value is not accurate based depth oscillations at the end of the sound speed range. Although our result is much different from the profile predicted on the basis of the geology in the area, it is in general agreement with a result obtained perturbatively by S. D. Rajan (pers. com. 1986).

\subsection{Future work}

A number of issues related to this work need to be addressed more fully in the future. We have largely ignored the discontinuity in sound speed at the water/sediment interface. In Chapter 4 we tried treating the low order (large angle of incidence) modes as being critically reflected at the interface, but this made the agreement between the inversion and the true profile worse than ignoring the presence of the discontinuity. The manner in which discontinuities in the index of refraction profile should be treated in the context of WKB theory, and the effect of the discontinuities on the cumulative phase needs further investigation.

In Chapter 4 we alluded to use of the inversions to estimate the density profile as well as the compressional wave speed profile. If the effects of density (assumed to be a function of depth only) are included, the homogeneous Helmholtz equation becomes [8]

$$
\nabla^{2} p(r, z)-\frac{1}{\rho(z)} \nabla \rho(z) \cdot \nabla p(r, z)+k^{2}(z) p(r, z)=0
$$

and separation of variables gives for the depth equation

$$
\frac{d^{2}}{d z^{2}} P(z)+\rho(z) \frac{d}{d z}\left(\frac{1}{\rho(z)}\right) \frac{d}{d z} P(z)+\left(k^{2}(z)-k_{r}^{2}\right) P(z)=0 .
$$

Substituting $v(z)=P(z) / \sqrt{\rho(z)}$ gives the Schrödinger type equation [36]

$$
\frac{d^{2}}{d z^{2}} v(z)+\left(k^{2}(z)+\mu(z)-k_{r}^{2}\right) v(z)=0
$$

where

$$
\mu(z)=\frac{\rho^{1 / 2}(z)}{2} \frac{d}{d z}\left[\frac{1}{\rho^{3 / 2}(z)} \frac{d}{d z} \rho(z)\right] .
$$


The WKB phase integral is now

$$
k_{0} \int_{0}^{z_{t}}\left(n^{2}(z)+\frac{\mu(z)}{k_{0}}-\xi\right)^{1 / 2} d z
$$

An open question is whether we can obtain a depth profile for a new variable $\chi(z)=n^{2}(z)+$ $\mu(z) / k_{0}$ at two frequencies and then eliminate $n^{2}(z)$ from the pair of equations since the index of refraction is frequency independent. This would leave an equation for $\mu(z)$ which through equation (5.4) provides a differential equation for $\rho(z)$. The numerical modelling program used in this work required constant densities in the sediments and the basement and did not provide a means to pursue the density question.

The inverse method was applied to one data set which had only six good eigenvalues. Identification of existing experimental data from other experiments obtained with arrays capable of resolving a larger number of modes (at least 10 to 15) would provide an opportunity to more fully test the inversions with experimental data. If data is available at two frequencies, use of Prony's method [15] to find the eigenvalues will provide an estimate of the modal attenution in adition to the eigenvalues. Using these inversion methods in conjunction with Prony's method will allow a geoacoustic model incorporating sediment sound speed, density, and attenuation to be developed. 


\section{Bibliography}

[1] MATH/LIBRARY: FORTRAN Subroutines for Mathematical Application, version 1.0, IMSL, Inc. Houston, TX . April 1987.

[2] Milton Abramowitz and Irene A. Stegun, editors. Handbook of Mathematical Functions. Dover Publications, Inc., New York, 1972.

[3] Daljit S. Ahluwalia and Joseph B. Keller. Exact and asymptotic representations of the sound field in a stratified ocean. In Joseph B. Keller and John S. Papadakis, editors, Wave Propagation in Underwater Acoustics, Springer-Verlag, New York, 1978.

[4] Kendall E. Atkinson. An Introduction to Numerical Analysis. John Wiley \& Sons, New York, 1978.

[5] Carl M. Bender and Steven A. Orszag. Advanced Mathematical Methods for Scientists and Engineers. McGraw-Hill Book Company, 1978.

[6] C. Allan Boyles. Acoustic Waveguides applications to oceanic science. John Wiley \& Sons, New York, 1984.

[7] L. Brekhovskikh and Yu Lysanov. Fundamentals of Ocean Acoustics. Springer-Verlag, New York, 1982.

[8] L.M. Brekhovskikh. Waves in Layered Media, second edition. Academic Press, Inc., New York, 1980.

[9] Paul J. Bushong. Tomographic measurement of barotropic motions. Engineer's Thesis, MIT/WHOI Joint Program, Cambridge, MA and Woods Hole, MA, August 1987. 
[10] K. Chadan and P.C. Sabatier. Inverse Problems in Scattering Theory. Springer-Verlag, New York, 1977.

[11] Clarence S. Clay and Herman Medwin. Acoustical Oceanography: Principles and Applications. John Wiley \& Sons, 1977.

[12] B. Cornuelle, C. Wunsch, D. Behringer, T. Birdsall, M. Brown, R. Heinmiller, R. Knox, K. Metzger, W. Munk, J Spiesberger, R. Spindel, D. Webb, and P. Worcester. Tomographic maps of the ocean mesoscale. part i: pure acoustics. J. Phys. Oceanogr., 15(2), 1985.

[13] Peter Craven and Grace Wahba. Smoothing noisy data with spline functions. Numer. Math., 31:377-403, 1979.

[14] Carl de Boor. A Practical Guide to Splines. Springer-Verlag, New York, 1978.

[15] Ferdinand S. Diemer. A Prony algorithm for shallow water waveguide analysis. Engineer's Thesis, MIT/WHOI Joint Program, Cambridge, MA and Woods Hole, MA, August 1987.

[16] J. I. Ewing. Elementary theory of seismic refraction and reflection measurements. In M. N. Hill, editor, The sea: ideas on progress in the study of the seas; volume 3, The earth beneath the sea, history, Interscience Publishers, New York, 1963.

[17] S. M. Flatte and R. B. Stoughton. Theory of acoustic measurements of internal wave strength as a function of depth, horizontal position and time. J. Geophys. RES, 91(C6):1709-1732, 1986.

[18] The PORT mathematical subroutine library. 1976.

[19] George V. Frisk, James A. Doutt, and Earl E. Hays. Geoacoustic models for the Icelandic Basin. J. Acoust. Soc. Am., 80(2):591-600, 1986.

[20] George V. Frisk and James F. Lynch. Shallow water waveguide characterization using the Hankel transform. J. Acoust. Soc. Am., 76(1):205-216, 1984. 
[21] George V. Frisk, James F. Lynch, and James A. Doutt. The determination of geoacoustic models in shallow water. In Tuncay Akal and Jonathan M. Berkson, editors, Ocean Seismo-Acoustics, pages 693-702, Plenum Publishing Corporation, New York, 1986.

[22] Nanny Fröman and Per Olaf Fröman. JWKB Approximation: contributions to the theory. North-Holland Publishing Company, Amsterdam, 1965.

[23] Gerald J. Fryer. Reflectivity of the ocean bottom at low frequency. J. Acoust. Soc. Am., $63(1): 35-42,1978$.

[24] K.M. Guthrie and C.T. Tindle. Ray effects in the normal mode approach in underwater acoustics. Journal of Sound and Vibration, 47(3):403-413, 1976.

[25] Edwin L. Hamilton. Geoacoustic modeling of the sea floor. J. Acoust. Soc. Am., $68(5): 1313-1340,1980$.

[26] H. Hochstadt and B. Lieberman. An inverse Sturm-Liouville problem with mixed given data. SIAM J. Appl. Math., 34:676-680, 1978.

[27] Finn B. Jensen and Melchiorre C. Ferla. SNAP: the SACLANTCEN normal-mode acoustic propagation model. SACLANTCEN memorandum SM-121, January 1979.

[28] Ram P. Kanwal. Linear Integral Equations: Theory and Technique. Academic Press, New York, 1971.

[29] N. Levinson. The inverse Sturm-Liouville problem. Mat. Tidsskr., 3:25-30, 1949.

[30] James F. Lynch, Subramaniam D. Rajan, and George V. Frisk. A perturbative inverse method for the determination of geoacoustic parameters in shallow water. In Harold $\mathrm{M}$. Merkinger, editor, Progress in Underwater Acoustics, Plenum Publishing Corporation, 1987.

[31] J. E. Matthews, P.J. Bucca, and W. H. Geddes. Preliminary environmental assessment of the PROJECT GEMINI site- Corpus Christi, Texas. Naval Ocean Research and Development Activity Report 120, June 1985. 
[32] Joyce R. McGlaughlin. Analytical methods for recovering coefficients in differential equations from spectral data. SIAM Review, 28(1):53-72, 1986.

[33] William Menke. Geophysical Data Analysis:Discrete Inverse Theory. Academic Press,Inc., New York, 1984.

[34] James H. Miller. Estimation of sea surface wave spectra using acoustic tomography. DSc thesis,MIT/WHOI Joint Program, Cambridge, MA and Woods Hole, MA, 1987.

[35] Walter Munk and Carl Wunsch. Ocean acoustic tomography: rays and modes. Reviews of Geophysics and Space Physics, 21(4):777-793, 83.

[36] Subramaniam D. Rajan. An inverse method for obtaining the attenuation prfile and small variations in the sound speed and density profiles of the ocean bottom. $\mathrm{PhD}$ thesis, MIT/WHOI Joint Program, Cambridge, MA and Woods Hole, MA, May 1985.

[37] Subramaniam D. Rajan, James F. Lynch, and George V. Frisk. Perturbative inversion methods for obtaining bottom geoacoustic parameters in shallow water. J. Acoust. Soc. Am., 82(3):998-1017, 1987.

[38] Christian H. Reinsch. Smoothing by spline functions. Numer. Math., 10:177-183, 1967.

[39] Bertram Ross. A brief history and exposition of the fundamental theory of fractional calculus. In Fractional Calculus and Its Applications; Proceedings of the International Conference held at the University of New Haven, June,1974, Springer-Verlag, New York, 1975.

[40] Francis P. Shepard. Submarine Geology. Harper \& Row, Publishers, New York, third edition edition, 1973.

[41] C.T. Tindle and K.M. Guthrie. Rays as interfering modes in underwater acoustics. Journal of Sound and Vibration, 34(2):291-295, 1974.

[42] H. Überall and N. C. Nicholas. Range focusing in a deep-ocean sound channel with parabolic profile. J. Acoust. Soc. Am., 44(5):1259-1261, 1968. 
[43] M. O. Vassell. Direct technique for calculating dielectric permittivity profiles from the distribution of mode indices in waveguides. J. Opt. Soc. Am., 65(9):1019-1021, 1975.

[44] John Archibald Wheeler. Semi classical analysis illuminates the connection between potential and bound state scattering. In E.H. Lieb, B. Simon, and A. S. Wightman, editors, Studies in Mathematical Physics: essays in honor of Valentine Bargmann, Princeton University Press, Princeton, New Jersey, 1976.

[45] Erich Zauderer. Partial Differential Equations of Applied Mathematics. John Wiley \& Sons, New York, 1983. 